Ann. I. H. Poincaré - PR 37, 4 (2001) 421-479

(C) 2001 Éditions scientifiques et médicales Elsevier SAS. All rights reserved

S0246-0203(01)01080-9/FLA

\title{
COUPLED MAP LATTICES WITH ASYNCHRONOUS UPDATINGS
}

\author{
Torsten FISCHER ${ }^{1}$ \\ Interdisziplinäres Zentrum für Wissenschaftliches Rechnen, Im Neuenheimer Feld 368, \\ 69120 Heidelberg, Germany
}

Received 30 August 1999, revised 19 February 2001

ABStRACT. - We consider on $M=\left(S^{1}\right)^{\mathbb{Z}^{d}}$ a family of continuous local updatings, of finite range type or Lipschitz-continuous in all coordinates with summable Lipschitz-constants. We show that the infinite-dimensional dynamical system with independent identically Poissondistributed times for the individual updatings is well-defined. We then consider the setting of analytically coupled uniformly expanding, analytic circle maps with weak, exponentially decaying interaction. We define transfer operators for the infinite-dimensional system, acting on Banach-spaces that include measures whose finite-dimensional marginals have analytic, exponentially bounded densities. We prove existence and uniqueness (in the considered Banachspace) of a probability measure and its exponential decay of correlations. ( 2001 Éditions scientifiques et médicales Elsevier SAS

RÉSUMÉ. - On considère sur $M=\left(S^{1}\right)^{\mathbb{Z}^{d}}$ une famille de mises-à-jour continues et locales, de type distance finie ou Lipschitzienne-continue sur toutes les coordonnées, les constantes Lipschitziennes étant de somme finie. On montre que le système dynamique à dimension infinie avec une distribution de Poisson identique et indépendante des instants de mise-à-jour est bien défini. Ensuite on considère le cas des applications du circle, analytiques, couplées entre elles analytiquement et à expension uniforme, à faible interaction exponentiellement décroissante. On définit des opérateurs de transfert pour le système à dimension infinie, agissant sur des espaces de Banach incluant des mesures dont les projections à dimensions finies ont des densités analytiques bornées exponentiellement. On montre l'existence et l'unicité (dans l'espace de Banach considéré) d'une mesure probabiliste et la décroissance exponentielle de ses corrélations. () 2001 Éditions scientifiques et médicales Elsevier SAS

E-mail address: torsten.fischer@iwr.uni-heidelberg.de (T. Fischer).

${ }^{1}$ The largest part of work on this paper was done at the University of Warwick and supported by the EC via TMR-Fellowship ERBFMBICT-961157. The paper was completed at the Niels Bohr Institute in Copenhagen. 


\section{Introduction}

In this paper we study coupled map lattices with indepentent identically (i.i.) Poissondistributed updatings at the individual sites.

A deterministic coupled map lattice (CML) is given by a $\mathbb{Z}^{d}$-lattice with a copy of the same Riemannian manifold at each lattice point (i.e. the state space is the product of these manifolds with index set $\mathbb{Z}^{d}$ ) and a map on the infinite space that can be decomposed into an uncoupled map that acts individually on each component and an 'interaction step' where the change of each coordinate depends also on the other sites.

L.A. Bunimovich and Y.G. Sinai prove in [8] (cf. also the remarks on this in [5]) the existence and uniqueness of an invariant measure and its exponential decay of correlations for a one-dimensional lattice of interval maps with weak coupling. By constructing a Markov partition they relate the system to a two-dimensional spin system whose Gibbs measure corresponds to the invariant measure of the CML.

G. Keller and M. Künzle prove in [21] the existence and uniqueness of an invariant measure for periodic or infinite one-dimensional lattices of weakly coupled interval maps by studying the transfer operators on the space of measures whose finite-dimensional marginals have densities of bounded variation. For small perturbation of the uncoupled map any invariant measure is 'close' to the one they found.

J. Bricmont and A. Kupiainen extend in [4] and [5,6] the result of Bunimovich and Sinai [8] to $\mathbb{Z}^{d}$-lattices of weakly coupled circle maps with analytic and Höldercontinuous interaction, respectively.

They represent the iterates of the Perron-Frobenius operator for finite-dimensional subsystems (over $\Lambda \subset \mathbb{Z}^{d}$ ) by a 'polymer'- or 'cluster'-expansion that gives rise to a representation of the corresponding invariant measure in terms of a $(d+1)$-dimensional spin system. The weak limit (as $\Lambda \rightarrow \mathbb{Z}^{d}$ ) of these measures is the unique (in a certain class) invariant probability measure and it is exponentially mixing with respect to (w.r.t.) spatio-temporal shifts.

C. Maes and A. Van Moffaert introduce in [25] for a similar setting as in [4] a simplified 'cluster'-expansion for the truncated Perron-Frobenius operator and show stochastic stability of the CML under stochastic perturbation.

In [2] V. Baladi, M. Degli Esposti, S. Isola, E. Järvenpää and A. Kupiainen define Frechet spaces, and, for $d=1$, a Banach space and transfer operators for the infinitedimensional systems, considered by Bricmont and Kupiainen in [4], and study the spectral properties of these operators.

In [13] we consider analytically coupled circle maps (uniformly expanding and analytic) on the $\mathbb{Z}^{d}$-lattice with exponentially decaying interaction and introduce Banach spaces for the infinite-dimensional system that include measures whose finitedimensional marginals have analytic, exponentially bounded densities. We define transfer operators on these spaces, get a unique (in the considered Banach spaces) probability measure and prove its exponential decay of correlations.

CMLs with multi-dimensional local systems of hyperbolic type have been studied by Ya.B. Pesin and Ya.G. Sinai [26], M. Jiang [17,18], M. Jiang and A. Mazel [19], M. Jiang and Ya.B. Pesin [20] and D.L. Volevich [29,30]. 
For detailed reviews on mathematical results on CMLs we refer to [2], [5], [7] and [20].

An interacting particle system (IPS) is given by an infinite lattice with a copy of the same state space (that is usually a finite or countable set but can also be a Riemannian manifold) at each site. The updating at an individual site is a deterministic or stochastic map (e.g. in the case of finite local state spaces it is given by a stochastic matrix with transition probabilities as its coefficients) that is applied with 'exponential waiting times', i.e. like the waiting times for jumps in a Poisson process. The jump rates for the updating depends also on the other sites. R.J. Glauber introduces in [14] (a special case of) the stochastic Ising model as a model for magnetism. The total state space $\{-1,+1\}^{\mathbb{Z}^{d}}$ represents the spins of the atoms at all sites. The rate for a flip of an individual spin depends on the states of the neighbour sites. F. Spitzer [27,28] and R.L. Dobrushin $[9,10]$ study more general systems where the individual jump rates do not only depend on the nearest neighbours.

A basic problem is to establish the existence of infinite systems with asynchronous updatings. The infinitely many jumps in a finite time-interval cannot be 'listed', i.e. there is no order preserving bijection between the time-ordered set of jumps and $\mathbb{N}$.

R.L. Dobrushin obtains in [9] the infinite system as the limit of subsystems over finitely many sites.

By using a percolation argument T.E. Harris proves in [15] that for systems of finite range interaction and a sufficiently small time interval the history of an individual particle depends on only finitely many sites, and so he provides a natural definition of the infinite system. With probability 1 the set $\mathbb{Z}^{d}$ splits into finite clusters such that each site is affected at most by sites in the same cluster.

R. Holley shows in [16] for generators, corresponding to one-dimensional models, and T.M. Liggett in [23] for the d-dimensioanal case, that these operators generate, in fact, a semigroup, acting on continuous functions.

Here we have only mentioned different methods to establish the existence of the infinite systems. For detailed reviews on IPSs and results on invariant measures, mixing properties, phase transitions and applications to physics and other sciences we refer to [11] and [24].

In this paper we consider the infinite topological product $M=\left(S^{1}\right)^{\mathbb{Z}^{d}}$ and continuous updating maps for the individual coordinates that are of finite range or Lipschitzcontinuous w.r.t. all coordinates with a summable family of Lipschitz constants (cf. Section 2.2 for the definition). The times for the updatings at the individual sites are independently Poisson-distributed with the same constant rate $\lambda>0$. For the finite range case we show that with probability 1 the updatings at any finite set of sites and any finite time-interval depend on only finitely many sites. Our proof uses time- and space-oriented percolation and is different from the one in [15]. This result provides a natural definition of the infinite dynamical system.

For the systems with infinite range interaction we show that with probability 1 it is well-defined as the net-limit of its finite-dimensional subsystems with arbitrary boundary conditions. We combine standard estimates for error growth with ideas from percolation theory. The limit of the corresponding Markov kernels, acting on continuous functions, 
exists and provides a definition of the infinite process, different from the widely used generator approach.

Our proofs still work if we replace $S^{1}$ by any compact Riemannian manifold or stochastic systems with finite state spaces. The assumption of having the same constant jump rate at all sites is by no means essential and can be weakened to the case of upper bounded individual jump rates that depend on other states as well. However, we do not consider these generalizations in this paper.

In a setting similar to that of [13], i.e. for analytically coupled circle maps (uniformly expanding and analytic) on the $\mathbb{Z}^{d}$-lattice with weak, exponentially decaying interaction but with asynchronous updatings as described above, we define transfer operators for the Markov kernels of the infinite system. The operators act on the Banach space $\mathcal{H}_{\vartheta}$ (introduced in [13]) that includes measures whose finite-dimensional marginals have analytic, exponentially bounded densities. Using 'cluster-expansion'-like techniques, we represent these integral operators in terms of configurations and prove the existence and uniqueness (in $\mathcal{H}_{\vartheta}$ ) of an invariant probability measure and its exponential decay of correlations.

The paper is organized as follows. Section 1 provides definitions, notation and some propositions about stochastic processes and metric spaces. In Section 2 we define the infinite-dimensional systems for finite range (Section 2.1) and infinite range interaction (Section 2.2) and the corresponding Markov kernels (Section 2.3). In Section 3 we study the transfer operators for a specific class of interactions. In Section 4 we prove the mixing properties of the invariant measure (found in Section 3) w.r.t. spatio-temporal shifts.

\section{Basic definitions and examples}

In this section we present definitions from probability theory and topology and also introduce most of the notation used in this paper. We have taken most definitions and statements on probability theory from [3].

DEFINITION 1.1. - $\mathbb{N}$ denotes the set of natural numbers including zero. Let $\left(E, \mathcal{A}_{2}\right)$ be a measurable space, $\left(\Omega, \mathcal{A}_{1}, P\right)$ a probability space and $\left(X_{t}\right)_{t \in I}$ a family (with index set $I \neq \emptyset)$ of random variables on $\left(\Omega, \mathcal{A}_{1}, P\right)$ with values in $E$.

- Then $\left(\Omega, \mathcal{A}_{1}, P,\left(X_{t}\right)_{t \in I}\right)$ is called a stochastic process with values in $\left(E, \mathcal{A}_{2}\right)$.

- If $I=\mathbb{R}^{\geqslant 0},[0, T]$ or $[0, T)$ for some $T>0$ the process is called a continuous time stochastic process.

- For fixed $\omega \in \Omega$ the map $t \mapsto X_{t}(\omega)$ is called the trajectory of $\omega$. It is also denoted by $X .(\omega)$.

- We consider the set $\mathbb{N}$ as measurable space with the discrete $\sigma$-algebra. For any set $\Lambda$ we denote by $\mathbb{N}^{\Lambda}$ the product space, equipped with the product $\sigma$-algebra.

A continuous time stochastic process with values in $\mathbb{N}^{\Lambda}$ and with index set I and $P$-a.a. of whose trajectories are non-decreasing (i.e. the functions $t \mapsto \pi_{q} \circ X_{t}(\omega)$ are non-decreasing for all $q \in \Lambda$ and $P$-a.a. $\omega \in \Omega$. ' $\pi_{q}$ ' denotes the projection on the qth coordinate.), is called a counting process with values in $\mathbb{N}^{\Lambda}$ We say that such a process is of finite expectation if for all $t \in I$ the random variable $\omega \mapsto \sum_{q \in \Lambda} \pi_{q} \circ X_{t}(\omega)$ has finite expectation. 
Remark 1.2.-

1. We will also use the short-hand-notation $X$. for a stochastic process if $\Omega, \mathcal{A}_{1}$ and $P$ are obvious from the context.

2. The term path seems to be more common than trajectory but we will denote something else later on by path.

3. Finite expectation means that with probability 1 there are only finitely many jumps (cf. Definition 1.3 below) in every finite time-interval.

Definition 1.3 (cf. [3]). - Let $\left(\Omega, \mathcal{A}_{1}, P,\left(X_{t}\right)_{t \in I}\right)$ be a continuous time counting process with values in $\mathbb{N}$ as in Definition 1.1 and $\omega \in \Omega$. We define

$$
\begin{aligned}
& X_{t}^{+}(\omega) \stackrel{\text { def }}{=} \begin{cases}X_{t}(\omega) & \text { if } I=[0, t], \\
\lim _{s \searrow t} X_{s}(\omega) & \text { otherwise, }\end{cases} \\
& X_{t}^{-}(\omega) \stackrel{\text { def }}{=} \begin{cases}\lim _{s \nearrow t} X_{s}(\omega) & t>0, \\
X_{0}(\omega) & t=0 .\end{cases}
\end{aligned}
$$

We say that $X .(\omega)$ jumps at time $t \geqslant 0$ if $X_{t}^{-}(\omega)<X_{t}^{+}(\omega)$. Then $X_{t}^{+}(\omega)-X_{t}^{-}(\omega)$ is called the size of the jump.

Let $X .(\omega)$ be a continuous time counting process with values in $\mathbb{N}^{\Lambda}$ and $\omega \in \Omega$. We say that $X .(\omega)$ jumps at time $t$ and site $q \in \Lambda$ if $\pi_{q} \circ X .(\omega)$ jumps at $t$. Then we also say that $\omega$ jumps at $(q, t)$.

We define the jump set $\Lambda(\omega, \mathbf{t})$ of $\omega$ at time $\mathbf{t}$ as the set of all $q \in \Lambda$ such that $\omega$ jumps at $(q, t)$.

Definition 1.4 (cf. [3]). - Let $I=\mathbb{R} \geqslant 0$ or $I=[0, T)$ for some $T>0$. A stochastic process $\left(\Omega, \mathcal{A}, P,\left(X_{t}\right)_{t \in I}\right)$ with values in $\mathbb{N}$ is called (normalized) Poisson process with parameter $\lambda>\mathbf{0}$ if the following holds:

1. The process has stationary and independent increments which for all $s<t \in I$ satisfy

$$
P\left(\left\{\omega: X_{t}(\omega)-X_{s}(\omega)=n\right\}\right)=p_{\lambda}(t-s, n)
$$

with

$$
p_{\lambda}(t, n) . \stackrel{\text { def }}{=} \mathrm{e}^{-\lambda t} \frac{(\lambda t)^{n}}{n !} .
$$

2. P-almost every trajectory $X .(\omega)$ is a right-continuous, increasing function having at most jumps of size 1 .

3. At time 0 P-a.a. trajectories have value 0 :

$$
P\left(\omega: X_{0}(\omega)=0\right)=1 .
$$

Theorem 1.5 (cf. [3], Satz 41.2). - For any $\lambda>0$ and $I$ as in Definition 1.4 there exists a (normalized) Poisson process with parameter $\lambda$. Any two such processes are equivalent (i.e. if $X^{1}$. and $X^{2}$. are two such processes then for any finite sequence $t_{1}<\cdots<t_{n}$ in I the projections $\left(X_{t_{1}}^{1}, \ldots, X_{t_{n}}^{1}\right)$ and $\left(X_{t_{1}}^{2}, \ldots, X_{t_{n}}^{2}\right)$ have the same distribution).

Definition 1.6. - Let $\Lambda$ be a nonempty set and $\left(\Omega_{q}, \mathcal{A}_{q}, P_{q},\left(X_{t}^{q}\right)_{t \in I}\right)_{q \in \Lambda}$ be a family of stochastic processes with values in $\left(E_{q}, \mathcal{A}^{q}\right)$, respectively. We set 


$$
\begin{aligned}
& \stackrel{\text { def }}{=} \prod_{q \in \Lambda} \Omega_{q}, \\
& \tilde{\mathcal{A}} \stackrel{\text { def }}{=} \bigotimes_{q \in \Lambda} \mathcal{A}_{q}, \\
& \tilde{P} \stackrel{\text { def }}{=} \bigotimes_{q \in \Lambda} P_{q}, \\
& \mathcal{A} \stackrel{\text { def }}{=} \text { completion of } \tilde{\mathcal{A}} \text { w.r.t. } \tilde{P}, \\
& P \stackrel{\text { def }}{=} \text { extension of } \tilde{P} \text { to } \mathcal{A} \\
\text { and } \quad & X_{t} \stackrel{\text { def }}{=}\left(X_{t}^{q}\right)_{q \in \Lambda} .
\end{aligned}
$$

In (6) we mean the cartesian product of spaces, in (7) the product sigma-algebra and in (8) the product measure.

Then the process $\left(\Omega, \mathcal{A}, P,\left(X_{t}\right)_{t \in I}\right)$ with values in $\left(\prod_{q \in \Lambda} E_{q}, \bigotimes_{q \in \Lambda} \mathcal{A}^{q}\right)$ is called the product of the family of processes.

Remark 1.7. -

1. Products of stochastic processes as in Definition 1.6 exist. For example the existence of the non-completed product measure follows from standard measure theory (cf. [3].)

2. For non-empty, at most countable $\Lambda$ and a family (indexed by $\Lambda$ ) of Poisson processes two such products $X^{1}$. and $X^{2}$. are equivalent because for all $q \in \Lambda$ the Poisson processes $\pi_{q} \circ X^{1}$. and $\pi_{q} \circ X^{2}$. are equivalent (cf. Theorem 1.5). It follows from the definition of the product $\sigma$-algebra $\otimes_{q \in \Lambda} \mathcal{A}^{q}$ that $X_{\text {. }}^{1}$ and $X_{\text {. are }}^{2}$ equivalent.

DEFINITION 1.8. - Let $\lambda>0$ and $\Lambda$ a nonempty, at most countable set. A Poisson process on $\Lambda$ with parameter $\lambda$ is the product of a family, indexed by $\Lambda$, of Poisson processes with parameter $\lambda$.

Remark 1.9. -

1. For $\lambda>0$ the Poisson process on $\mathbb{Z}^{d}$ with parameter $\lambda$ is clearly not of finite expectation. In fact, for any $t>0$ there are $P$-almost surely infinitely many jumps in $[0, t]$, i.e.

$$
P\left(\left\{\omega: \sum_{q \in \mathbb{Z}^{d}} \pi_{q} \circ X_{t}(\omega)=\infty\right\}\right)=1 .
$$

2. But if $\Lambda_{1} \subset \mathbb{Z}^{d}$ is finite then $\pi_{\Lambda_{1}} \circ X$. $(\omega)$ has finitely many jumps in $[0, t]$ for $P$-a.a. $\omega \in \Omega$ and any $t>0$.

3. There are P-almost surely no simultaneous jumps at two different sites:

$$
P\left(\left\{\omega: \exists q_{1} \neq q_{2} \in \mathbb{Z}^{d}, t \geqslant 0 \text { such that } \omega \text { jumps at }\left(q_{1}, t\right) \text { and }\left(q_{2}, t\right)\right\}\right)=0 .
$$

4. For $0 \leqslant t_{0}<t$

$$
P\left(\left\{\omega: \omega \text { jumps at } t_{0}\right\}\right)=0 .
$$


Proof of Remark 1.9. - We only show (13). The proofs of the other statements are similar. We set

$$
A\left(q_{1}, q_{2}, T\right) \stackrel{\text { def }}{=}\left\{\omega: \exists t \in[0, T) \text { such that } \omega \text { jumps at }\left(q_{1}, t\right) \text { and }\left(q_{2}, t\right)\right\} .
$$

We have to prove that the set

$$
\bigcup_{T \in \mathbb{N}} \bigcup_{q_{1}, q_{2} \in \mathbb{Z}^{d}} A\left(q_{1}, q_{2}, T\right)
$$

has $P$-measure zero and it is sufficient to show that

$$
P\left(A\left(q_{1}, q_{2}, T\right)\right)=0
$$

for fixed $q_{1} \neq q_{2} \in \mathbb{Z}^{d}$ and $T>0$. For this we set

$$
I_{N, k} \stackrel{\text { def }}{=}\left[(k-1) \frac{T}{N}, k \frac{T}{N}\right)
$$

for $N \in \mathbb{N} \backslash\{0\}$ and $1 \leqslant k \leqslant N$. By (4) we have for $i=1,2$ :

$$
P\left(\left\{\omega: \text { jumps at }\left(q_{i}, t\right) \text { for some } t \in I_{N, k}\right\}\right)=1-\mathrm{e}^{-\lambda \frac{T}{N}}
$$

and so, using the estimate $\mathrm{e}^{x} \geqslant 1+x$ :

$$
\begin{aligned}
& P\left(\left\{\omega: \exists k ; t_{1}, t_{2} \in I_{N, k} \text { such that } \omega \text { jumps at }\left(q_{1}, t_{1}\right) \text { and }\left(q_{2}, t_{2}\right)\right\}\right) \\
& \quad \leqslant N\left(1-\mathrm{e}^{-\lambda \frac{T}{N}}\right)^{2} \leqslant \lambda^{2} T^{2} \frac{1}{N}
\end{aligned}
$$

which converges to 0 as $N \rightarrow \infty$.

The following two definitions prepare Definition 1.13 that we will need in Section 2.

DEFINITION 1.10. - In view of Definition 1.4 and Remark 1.9 we define (for a given Poisson process like in that remark) the set $\mathcal{N}_{1}$ of $P$-measure zero:

$\mathcal{N}_{1} \stackrel{\text { def }}{=}\{\omega: X .(\omega)$ is not non-decreasing, has jumps at 0, simultaneous jumps or jumps of size greater than 1$\}$.

DEFINITION 1.11. - Let $\Lambda \subset \mathbb{Z}^{d}$. Then we denote its complement by $\Lambda^{C} \stackrel{\text { def }}{=} \mathbb{Z}^{d} \backslash \Lambda$.

DEFINITION 1.12. - For $q=\left(q_{1}, \ldots, q_{n}\right) \in \mathbb{Z}^{d}$ we define

$$
\|q\| \stackrel{\text { def }}{=}\left|q_{1}\right|+\cdots+\left|q_{n}\right| .
$$

For $R \geqslant 0$

$$
B_{R}(q) \stackrel{\text { def }}{=}\left\{\tilde{q} \in \mathbb{Z}^{d}:\|q-\tilde{q}\| \leqslant R\right\}
$$

is the set of points that have distance at most $R$ from $q$. 
DEFINITION 1.13. - Let $a, b \in \mathbb{Z}^{d}$ and $n \geqslant 0$. A path from $a$ to $b$ is a finite sequence $Q=\left(q_{0}=a, q_{1}, \ldots, q_{n}=b\right)$ of points $q_{i} \in \mathbb{Z}^{d}$. We call $\sum_{i=1}^{n}\left\|q_{i}-q_{i-1}\right\|$ the length and $\max _{0 \leqslant i \leqslant n-1}\left\|q_{i+1}-q_{i}\right\|$ the step size of $Q$. Note the special case of a path $Q=\left(q_{0}\right)$. It is called the empty path at site $q_{0}$ and we define both its length and step size to be 0 .

Definition 1.14. - Let $\left(\Omega, \mathcal{A}, P,\left(X_{t}\right)_{t \geqslant 0}\right)$ be a Poisson process with parameter $\lambda>0$ and with values in $\mathbb{N}^{\mathbb{Z}^{d}}$. Let $T>0, \underset{\widetilde{Q}}{\omega} \Omega$ and $Q=\left(q_{0}=a, q_{1}, \ldots, q_{n}=b\right) a$ path. We extend $Q$ to the infinite sequence $\widetilde{Q}=\left(q_{0}, q_{1}, \ldots, q_{n}, q_{n+1}=q_{n}, \ldots\right)$ in which $q_{n}$ is repeated.

We define a process $\left(\Omega, \mathcal{A}, P,\left(Z_{t}\right)_{t \in[0, T]}\right)$ with values in $\mathbb{N}$ as follows.

$$
\begin{aligned}
Z:[0, t] \times \Omega & \rightarrow \mathbb{N}, \\
(t, \omega) & \mapsto Z_{t}(\omega) .
\end{aligned}
$$

If $\omega \in \mathcal{N}_{1}$ or it does not jump at $\left(q_{0}, t\right)$ for any $t \in(0, T)$ we set $Z .(\omega)=0$ on $[0, T]$.

Otherwise there is a maximal sequence

$$
t_{-1} \stackrel{\text { def }}{=} T>t_{0}>t_{1}>\cdots>t_{m(\omega)}
$$

such that

$$
t_{i} \stackrel{\text { def }}{=} \max \left\{t \in\left(0, t_{i-1}\right): \text { jumps at }\left(q_{i}, t\right)\right\} \quad \text { for } 0 \leqslant i \leqslant m(\omega) .
$$

'Maximal' means that $\omega$ does not jump at $q_{m(\omega)+1}$ in the time interval $\left(0, t_{m(\omega)}\right)$ and so the sequence (25) cannot be extended. (Intuitively one can think that one sits at time $T$ at site $q_{0}$ and, going backwards in time, waits for the next jump of $\omega$ at $q_{0}$ (which happens at time $t_{0}$ ), then jumps (instantly) to $q_{1}$ and waits (backwards in time) for the next jump of $\omega$ at $q_{1}$, then jumps to $q_{2}$ etc. After $n$ jumps (should this occur) one does not change the sites any more, but possibly jumps from $q_{n}$ to $q_{n} . m(\omega)$ is the total number of jumps. It is $P$-a.s. finite because $P$-a.a. $\omega$ have only finitely many jumps at $q_{n}$.)

We set for $t \in[0, T]$ :

$$
\widetilde{Z}_{t}(\omega) \stackrel{\text { def }}{=} \begin{cases}i & \text { for } t \in\left[t_{i}, t_{i-1}\right) \\ m(\omega) & \text { for } t \in\left[0, t_{m(\omega)}\right]\end{cases}
$$

And $Z_{.}(\omega)$ is the (uniquely defined) right-continuous function, such that $Z_{.}(\omega)=$ $\widetilde{Z}_{T-.}(\omega)$ everywhere, except possibly where these functions jump. Then $(\Omega, \mathcal{A}, P$, $\left(Z_{t}\right)_{t \in[0, T]}$ ) is a Poisson process with parameter $\lambda$. (A precise proof of this uses that the constructed process is 'made of' independent Poisson processes and that these have independent increments.) We call it the Poisson process induced by the path $Q$.

DEFINITION 1.15. - In the setting of Definition 1.14 we call $Q$ a causal path w.r.t. $(t, \omega)$ if $Z_{T}(\omega) \geqslant n$ and a maximal causal path w.r.t. $(t, \omega)$ if $Z_{T}(\omega)=n$. (The latter means that $Q=\left(q_{0}, \ldots, q_{n}\right)$ cannot be extended to any causal path $\left(q_{0}, \ldots, q_{n}, q_{n+1}\right)$.)

We define:

- Path $(q, n, R)$ to be the set of paths that start at $q$, have exactly $n$ steps and are of step size at most $R$. 
- Path $(q \rightarrow \Lambda)$ for any $\emptyset \neq \Lambda \in \mathbb{Z}^{d}$ to be the set of paths starting at $q$ and ending in $\Lambda$.

- Path $_{C}(t, \omega, q, \Lambda)$ for $q \in \Lambda$ to be the set of causal w.r.t. $(t, \omega)$ paths $Q=\left(q_{0}=\right.$ $\left.q, \ldots, q_{n}\right)$ such that

1. $Q$ is maximal causal and $q_{0}, \ldots, q_{n} \in \Lambda$, or

2. $q_{0}, \ldots, q_{n-1} \in \Lambda$ and $q_{n} \in \Lambda^{C}$.

- Path $_{c}\left(t, \omega, q \rightarrow \Lambda^{C}\right)$ for $q \in \Lambda$ to be the set of causal paths $\left(q_{0}=q, \ldots, q_{n}\right)$ such that $q_{0}, \ldots, q_{n-1} \in \Lambda$ and $q_{n} \in \Lambda^{C}$. (So this is the subset of elements in $\operatorname{Path}_{c}(t, \omega, q, \Lambda)$ for which case 2. applies. $)$

Remark 1.16. -

1. We have defined the property of being causal for general paths and not related this definition to any kind of interaction. When we study finite range interaction, of range $R$ say, we will consider only causal paths of step size at most $R$.

2. A term like inverse causal path from $a$ to $b$ instead of causal path would actually be more appropiate as it corresponds to $b$ affecting $a$ (cf. Definition 2.1) but not necessarily the other way around. However, we prefer the shorter notion.

Definition 1.17 (cf. [3]). - Let $\left(\Omega_{1}, \mathcal{A}_{1}\right)$ and $\left(\Omega_{2}, \mathcal{A}_{2}\right)$ be measurable spaces. $A$ map $K: \Omega_{1} \times \mathcal{A}_{2} \rightarrow[0,1]$ is called a Markov kernel from $\left(\Omega_{1}, \mathcal{A}_{1}\right)$ to $\left(\Omega_{2}, \mathcal{A}_{2}\right)$ if the two following conditions are satisfied:

MK1 $\omega_{1} \mapsto K\left(\omega_{1}, A_{2}\right)$ is $\mathcal{A}_{1}$-measurable for all $A_{2} \in \mathcal{A}_{2}$.

MK2 $\quad A_{2} \mapsto K\left(\omega_{1}, A_{2}\right)$ is a probability measure on $\mathcal{A}_{2}$ for all $\omega_{1} \in \Omega_{1}$.

If $\left(\Omega_{1}, \mathcal{A}_{1}\right)=\left(\Omega_{2}, \mathcal{A}_{2}\right)$ then $K$ is called a Markov kernel on $\left(\Omega_{1}, \mathcal{A}_{1}\right)$.

Example 1.18. - Let $\left(Y, \varrho_{Y}\right)$ be a metric space and $\mathcal{B}_{Y}$ its Borel $\sigma$-algebra. $\mathcal{C}^{0}(Y, Y)$ is the space of continuous maps from $Y$ to $Y$. It has a uniform metric, defined by $\varrho_{\mathcal{C}^{0}(Y, Y)}\left(g_{1}, g_{2}\right)=\sup _{\mathbf{y} \in \mathbf{Y}} \varrho_{Y}\left(g_{1}(\mathbf{y}), g_{2}(\mathbf{y})\right)$ and the Borel $\sigma$-algebra $\mathcal{B}_{\mathcal{C}^{0}(Y, Y)}$ w.r.t. this metric. Further, let $(\Omega, \mathcal{A}, P)$ be a probability space and

$$
\begin{aligned}
S: \Omega & \rightarrow \mathcal{C}^{0}(Y, Y), \\
\omega & \mapsto S_{\omega},
\end{aligned}
$$

a measurable (w.r.t. the $\sigma$-algebras $\mathcal{A}$ and $\mathcal{B}_{\mathcal{C}^{0}(Y, Y)}$ ) map.

Then

$$
K_{S}\left(y, Y_{1}\right) \stackrel{\text { def }}{=} P\left(\left\{\omega: S_{\omega}(y) \in Y_{1}\right\}\right)
$$

for all $y \in Y, Y_{1} \in \mathcal{B}_{Y}$, defines a Markov kernel on $\left(Y, \mathcal{B}_{Y}\right)$.

Proof. - To verify MK1 we fix an $Y_{1} \in \mathcal{B}_{Y}$ and show that the map $y \mapsto K_{S}\left(y, Y_{1}\right)$ is measurable. First we note that $S$ can be seen as a measurable map from $\Omega \times Y$ to $Y$. We write it as the composite of measurable maps $S \times \operatorname{id}_{Y}$ and the 'evaluation map':

$$
(\omega, y) \mapsto\left(S_{\omega}, y\right) \mapsto S_{\omega}(y) .
$$

The map $S \times \operatorname{id}_{Y}$ is measurable by assumption and the definition of the product $\sigma$-algebra of $\mathcal{C}^{0}(Y, Y) \times Y$. The evaluation map is continuous (w.r.t. the product topology), hence 
measurable w.r.t. the Borel $\sigma$-algebras. So the composite in (30) is measurable in $\Omega \times M$. It follows that the map $y \mapsto P\left(\left\{\omega: S_{\omega}(y) \in Y_{1}\right\}\right)$ is measurable (cf. Lemma 8.1 on p. 159 in [22]) and so MK1 holds.

Next we show MK2. Consider for fixed $y \in Y$ the composite of measurable maps

$$
\omega \mapsto(\omega, y) \mapsto S_{\omega}(y)
$$

that maps $\Omega$ to $Y$. We see that $K(y, \cdot)$ is the image of $P$ w.r.t. this map and so a probability measure which was to be shown.

DEFINITION 1.19 (cf. [3]). - Let $K$ be a Markov kernel from $\left(\Omega_{1}, \mathcal{A}_{1}\right)$ to $\left(\Omega_{2}, \mathcal{A}_{2}\right)$ and $E^{*}\left(\mathcal{A}_{i}\right)(i=1,2)$ the set of $\mathcal{A}_{i}$-measurable functions with values in $[0, \infty]$. Then $K$ defines a map from $E^{*}\left(\mathcal{A}_{2}\right)$ to $E^{*}\left(\mathcal{A}_{1}\right)$ as follows:

$$
(K f)\left(\omega_{1}\right) \stackrel{\text { def }}{=} \int_{\Omega_{2}} K\left(\omega_{1}, \mathrm{~d} \omega_{2}\right) f\left(\omega_{2}\right)
$$

for any $f \in E^{*}\left(\mathcal{A}_{2}\right)$. The notation on the rhs of (32) means that $f$ is integrated w.r.t. the probabiltiy measure on $\Omega_{2}$ that is described in Definition 1.17, MK2.

Example 1.20 (cf. [3]). - For the characteristic function $\chi_{A_{2}}$ of an $\mathcal{A}_{2}$-measurable set $A_{2}$ we get

$$
K \chi_{A_{2}}\left(\omega_{1}\right)=K\left(\omega_{1}, A_{2}\right)
$$

Now we consider a special case of Example 1.18.

Example 1.21. - Let $S: Y \rightarrow Y$ be a continuous map on $\left(Y, \varrho_{Y}\right)$ and let $(\Omega, \mathcal{A}, P$, $\left.\left(X_{t}\right)_{t \in I}\right)$ be a counting process with values in $\mathbb{N}$ and $t \in I$.

The map

$$
\begin{aligned}
S_{\omega}^{t}: Y & \rightarrow Y, \\
y & \mapsto S^{X_{t}(\omega)}(y),
\end{aligned}
$$

where $S^{X_{t}(\omega)}$ denotes the $X_{t}(\omega)$ th iterate of $S$, is well-defined for all $\omega \in \Omega$. Further, $S_{\omega}(y)$ is measurable w.r.t. $(\omega, y)$. In fact, $S_{\omega}$ depends just on $X_{t}(\omega)$ and so we get a countable, measurable partition of $\Omega$ :

$$
\begin{gathered}
\Omega=\bigcup_{n \in \mathbb{N}} U(n), \\
\text { with } U(n) \stackrel{\text { def }}{=}\left\{\omega \in \Omega: X_{t}(\omega)=n\right\} .
\end{gathered}
$$

We define a Markov kernel by

$$
\begin{aligned}
K_{S}^{t}\left(y, Y_{1}\right) & \stackrel{\text { def }}{=} P\left(\left\{\omega: S_{\omega}^{t}(y) \in Y_{1}\right\}\right) \\
& =\sum_{n: S^{n}(y) \in Y_{1}} P(U(n)) \\
& =\int_{\Omega} \mathrm{d} P(\omega) \chi_{Y_{1}} \circ S_{\omega}^{t}(y)
\end{aligned}
$$


for $y \in Y$ and $Y_{1} \in \mathcal{B}_{Y}$.

We see that this Markov kernel acts on a measurable function $f: Y \rightarrow[0, \infty]$ by

$$
\left(K_{S}^{t} f\right)(y)=\sum_{n=0}^{\infty} P(U(n)) f\left(S^{n}(y)\right) .
$$

We prepare a generalization of Example 1.21 with a definition and a technical lemma.

DEFINITION 1.22. - Let $\mathcal{F}$ be the set of finite subsets of $\mathbb{Z}^{d}$. Consider a fixed $\Lambda \in \mathcal{F} \backslash\{\emptyset\}$. We define $\mathcal{J}$ to be the union of a one-point set $\left\{j_{\infty}\right\}$ and the set of finite sequences $\left(\Lambda_{1}, \ldots, \Lambda_{n}\right)$ of subsets of $\Lambda$. Then $\mathcal{J}$ is countable and we consider it as a measurable space, equipped with the discrete $\sigma$-algebra.

Let $\left(\Omega, \mathcal{A}, P,\left(X_{t}\right)_{t \in I}\right)$ be a continuous time counting process with values in $\mathbb{N}^{\Lambda}$ and index-set $I=[0, T)$ or $[0, T]$. We define a map

$$
\begin{aligned}
\mathbf{j}: \Omega & \rightarrow \mathcal{J} \\
\omega & \mapsto \mathbf{j}(\omega) .
\end{aligned}
$$

If $X .(\omega)$ is non-decreasing, has only finitely many jumps and at most jumps of size 1 then we define $\mathbf{j}(\omega)$ to be the (time-ordered) sequence of jump sets of $\omega$. Otherwise we set $\mathbf{j}(\omega)=j_{\infty}$. We define for $j \in \mathcal{J}$ :

$$
U(j) \stackrel{\text { def }}{=}\{\omega: \mathbf{j}(\omega)=j\}
$$

LEMmA 1.23. - Let $\Lambda \in \mathcal{F} \backslash\{\emptyset\}$ be fixed and $\left(\Omega, \mathcal{A}, P,\left(X_{t}\right)_{t \in I}\right)$ a continuous time counting process with index-set $I=[0, T)$ or $I=[0, T]$ and values in $\mathbb{N}^{\Lambda}$ such that for $P$-a.a. $\omega$ the trajectory $X .(\omega)$ is non-decreasing, has only finitely many jumps and at most jumps of size 1. Then the map $\mathbf{j}$, as defined in Definition 1.22, is measurable.

Proof. - We consider the case $I=[0, T]$. The case $I=[0, T)$ is analogous. By assumption $\mathcal{N}=U\left(j_{\infty}\right)$ is measurable and has measure zero. We have to show that $U(j)$ is measurable for any $j=\left(\Lambda_{1}, \ldots, \Lambda_{n}\right)$. For any $q_{1}, q_{2} \in \Lambda$ and $n_{1}, n_{2} \in \mathbb{N} \backslash\{0\}$ we define $A_{1}\left(q_{1}, n_{1}, q_{2}, n_{2}\right)$ to be the set of all $\omega \in \Omega \backslash \mathcal{N}$ that have at least $n_{1}$ jumps at $q_{1}$ and at least $n_{2}$ jumps at $q_{2}$ and the $n_{1}$ th jump at $q_{1}$ happens at the same time as the $n_{2}$ th jump at $q_{2}$. Similarly, $A_{2}\left(q_{1}, n_{1}, q_{2}, n_{2}\right)$ is the set of all $\omega \in \Omega \backslash \mathcal{N}$ that have at least $n_{1}$ jumps at site $q_{1}$ and the $n_{1}$ th jump at $q_{1}$ occurs before the $n_{2}$ th jump at $q_{2}$ (if there is an $n_{2}$ th jump at $q_{2}$ at all - if that is not the case then this second condition is automatically satisfied). We only show the measurability of the sets $A_{2}(\cdot)$. The proof of the measurability of the sets $A_{1}(\cdot)$ uses similar arguments.

$$
A^{\geqslant}\left(q_{1}, n_{1}, t\right) \stackrel{\text { def }}{=}\left\{\omega \in \Omega \backslash \mathcal{N}: \pi_{q_{1}} \circ X_{t}(\omega) \geqslant n_{1}\right\}
$$

is the set of all $\omega \in \Omega \backslash \mathcal{N}$ that have at least $n_{1}$ jumps at site $q_{1}$ and the $n_{1}$ th of these jumps happens at the latest at time $t$.

Analogously,

$$
A^{<}\left(q_{2}, n_{2}, t\right) \stackrel{\text { def }}{=}\left\{\omega \in \Omega \backslash \mathcal{N}: \pi_{q_{2}} \circ X_{t}(\omega)<n_{2}\right\}
$$


is the set of all $\omega \in \Omega \backslash \mathcal{N}$ with at most $n_{2}-1$ jumps at $q_{2}$ in the time interval $[0, t]$.

The sets $A^{\geqslant}\left(q_{1}, n_{1}, t\right)$ and $A^{<}\left(q_{2}, n_{2}, t\right)$ are measurable, and so is $A_{2}\left(q_{1}, n_{1}, q_{2}, n_{2}\right)$ since

$$
A_{2}\left(q_{1}, n_{1}, q_{2}, n_{2}\right)=\bigcup_{t \in[0, T] \cap \mathbb{Q}}\left(A^{\geqslant}\left(q_{1}, n_{1}, t\right) \cap A^{<}\left(q_{2}, n_{2}, t\right)\right) .
$$

Now $\omega$ belongs to $U(j)$ if and only if, for all $1 \leqslant k \leqslant n$ and $q_{1}, q_{2} \in \Lambda_{k}$ and $q_{3} \in \Lambda \backslash \Lambda_{k}$ the following holds:

- If for exactly $n_{1}$ indices $1 \leqslant i \leqslant k$ the point $q_{1}$ belongs to $\Lambda_{i}$ and for exactly $n_{2}$ indices $1 \leqslant j \leqslant k$ the point $q_{2}$ belongs to $\Lambda_{j}$ then $\omega \in A_{1}\left(q_{1}, n_{1}, q_{2}, n_{2}\right)$.

- If for exactly $n_{1}$ indices $1 \leqslant i \leqslant k$ the point $q_{1}$ belongs to $\Lambda_{i}$ and for exactly $n_{3}-1$ indices $1 \leqslant j<k$ the point $q_{3}$ belongs to $\Lambda_{j}$ then $\omega \in A_{2}\left(q_{1}, n_{1}, q_{3}, n_{3}\right)$.

- If for exactly $l \geqslant 0$ indices $1 \leqslant n_{1}<n_{2}<\cdots<n_{l} \leqslant n$ a point $q \in \Lambda$ belongs to $\Lambda_{n_{i}}$ then $\omega \in\left\{\tilde{\omega} \in \Omega \backslash \mathcal{N}: \pi_{q} \circ X_{T}(\tilde{\omega})=l\right\}$.

We see that $U(j)$ is the intersection of finitely many measurable sets and hence measurable.

Example 1.24. - We consider a generalization of Example 1.21. Let $\left(Y, \varrho_{Y}\right)$ be a measurable space, $\mathcal{Y}$ its Borel sigma-algebra, $\Lambda$ a non-empty finite set and $(\Omega, \mathcal{A}, P$, $\left.\left(X_{t}\right)_{t \in I}\right)$ a counting process with values in $\mathbb{N}^{\Lambda}$ that has finite expectation and with $P$ almost surely only jumps of size at most 1 . Let $S=\left(S_{\Lambda_{1}}\right)_{\Lambda_{1} \subset \Lambda}$ be a family of continuous maps on $Y^{\Lambda}$, such that $S_{\Lambda_{1}}$ changes at most the $\Lambda_{1}$-coordinates, i.e. if $\mathbf{y}_{\Lambda} \in Y^{\Lambda}$ and $q \in \Lambda \backslash \Lambda_{1}$ we have for the $q$ th coordinate $\pi_{q} \circ S_{\Lambda_{1}}\left(\mathbf{y}_{\Lambda}\right)=y_{q}$.

For $t \in I$ and $P$-a.a. $\omega \in \Omega$ with $X_{t}(\omega) \in \mathbb{N}^{\Lambda}$ we have a finite sequence of jump-sets $\mathbf{j}(\omega)=\left(\Lambda_{1}, \ldots, \Lambda_{n}\right)$, as defined in Definition 1.22, and it depends measurably on $\omega$, as was shown in Lemma 1.23. We define

$$
\begin{aligned}
S_{\omega}^{t}: Y^{\Lambda} & \rightarrow Y^{\Lambda}, \\
\mathbf{y}_{\Lambda} & \mapsto S_{\mathbf{j}(\omega)}\left(\mathbf{y}_{\Lambda}\right) \stackrel{\text { def }}{=} S_{\Lambda_{n}} \circ \cdots \circ S_{\Lambda_{1}}\left(\mathbf{y}_{\Lambda}\right) .
\end{aligned}
$$

We get a representation of $K_{S}^{t}\left(\mathbf{y}_{\Lambda}, Y_{1}\right)$, similar to the one in (37):

$$
\begin{aligned}
K_{S}^{t}\left(\mathbf{y}_{\Lambda}, Y_{1}\right) & =P\left(\left\{\omega: S_{\omega}^{t}\left(\mathbf{y}_{\Lambda}\right) \in Y_{1}\right\}\right) \\
& =\int_{\Omega} \mathrm{d} P(\omega) \chi_{Y_{1}} \circ S_{\omega}^{t}\left(\mathbf{y}_{\Lambda}\right) \\
& =\sum_{j \in \mathcal{J}: S_{j}\left(\mathbf{y}_{\Lambda}\right) \in Y_{1}} P(U(j))
\end{aligned}
$$

for $\mathbf{y}_{\Lambda} \in Y^{\Lambda}$ and $Y_{1} \in \bigotimes_{q \in \Lambda} \mathcal{Y}$.

We have seen in Example 1.24 that $S_{\omega}^{t}$ depends on $\mathbf{j}(\omega)$ only.

As we are interested in spatially extended systems we need some definitions and facts about infinite-dimensional systems.

DEFINITION 1.25. $-S^{1}$ is the one-dimensional sphere. We define it to be isometric as Riemannian manifold to $\mathbb{R} / 2 \pi \mathbb{Z}$. This defines in particular a metric $\varrho_{S^{1}}$ on $S^{1}$ and also the normalized Lebesgue measure on the (completed) Borel $\sigma$-algebra. 
The diameter of $S^{1}$ is

$$
c_{S} \stackrel{\text { def }}{=} \operatorname{diam}_{\varrho_{S 1}}\left(S^{1}\right)=\pi .
$$

(It seems a bit redundant to introduce the constant $c_{S}$ instead of using $\pi$ in the following. But we indicate that the proofs in Section 2 work if $S^{1}$ is replaced by any compact Riemannian manifold or more general by a bounded metric space with a Borel probability measure. Further, we use the letter ' $\pi$ ' as notation for projections.)

We set

$$
M \stackrel{\text { def }}{=}\left(S^{1}\right)^{\mathbb{Z}^{d}}
$$

and give it the product topology and product Lebesgue measure on the (completed) Borel $\sigma$-algebra.

For $\Lambda \subset \mathbb{Z}^{d}$ we denote by $\pi_{\Lambda}$ the projection on the $\Lambda$-coordinates.

Note that the product of the Borel $\sigma$-algebras is the same as the Borel $\sigma$-algebra for the product space. $M$ is compact and metrizable in the following way:

DEFINITION 1.26. - Let $(b(q))_{q \in \mathbb{Z}^{d}}$ be a family of positive numbers such that

$$
\lim _{R \rightarrow \infty} \sup _{\|q\| \geqslant R} b(q)=0 .
$$

Then the metric $\varrho_{M}$ on $M$, associated to $(b(q))_{q \in \mathbb{Z}^{d}}$, is defined by

$$
\varrho_{M}(\mathbf{x}, \mathbf{y}) \stackrel{\text { def }}{=} \sup _{q \in \mathbb{Z}^{d}} b(q) \varrho_{S^{1}}\left(x_{q}, y_{q}\right)
$$

for $\mathbf{x}, \mathbf{y} \in M$.

Remark 1.27.-

1. One can easily show that $\varrho_{M}$, as defined in Definition 1.26 , is in fact a metric and also compatible with the product topology.

2. A sequence $\left(\mathbf{x}^{(n)}\right)_{n \in \mathbb{N}}$ in $M$ converges w.r.t. the product topology iff it converges w.r.t. each coordinate, i.e. $\left(x_{q}^{(n)}\right)_{n \in \mathbb{N}}$ converges for every $q \in \mathbb{Z}^{d}$. The same holds also for nets $\left(\mathbf{x}^{\Lambda}\right)_{\Lambda \in \mathcal{F} \text {. }}$

3. The product topology does not distinguish any particular sites despite the fact that the weights $b(q)$ depend on $q$. Spatial shifts, like $\mathbf{x} \mapsto \tilde{\mathbf{x}}$ with $\tilde{x}_{q}=x_{q-r}$ for some $r \in \mathbb{Z}^{d}$, are homeomorphisms.

4. The space $\mathcal{C}^{0}(M, M)$ of continuous maps on $\left(M, \varrho_{M}\right)$ is complete w.r.t. the metric defined by

$$
\varrho_{\mathcal{C}^{0}(M, M)}(f, g) \stackrel{\text { def }}{=} \sup _{\mathbf{x} \in M} \varrho_{M}(f(\mathbf{x}), g(\mathbf{x})) .
$$

We denote by $\mathcal{B}_{\mathcal{C}^{0}(M, M)}$ the Borel $\sigma$-algebra w.r.t. this metric.

Lemma 1.28. - Let $(\Omega, \mathcal{A})$ be a measurable space and $\left(f^{\Lambda}\right)_{\Lambda \in \mathcal{F} \backslash\{\emptyset\}}$ be a net of measurable maps 


$$
\begin{aligned}
f^{\Lambda}: \Omega & \rightarrow \mathcal{C}^{0}(M, M), \\
\omega & \mapsto f_{\omega}^{\Lambda},
\end{aligned}
$$

such that for all $\Lambda_{1} \in \mathcal{F} \backslash\{\emptyset\}$ and $\omega \in \Omega$ the net $\left(\pi_{\Lambda_{1}} \circ f_{\omega}^{\Lambda}\right)_{\Lambda_{1} \subset \Lambda \in \mathcal{F}}$ converges (as $\left.\Lambda \rightarrow \mathbb{Z}^{d}\right)$ in $\mathcal{C}^{0}\left(M,\left(S^{1}\right)^{\Lambda_{1}}\right)$, say to $\pi_{\Lambda_{1}} \circ f_{\omega}$.

Then

$$
f_{\omega, q}(\mathbf{x}) \stackrel{\text { def }}{=} \lim _{\Lambda \rightarrow \mathbb{Z}^{d}} \pi_{q} \circ f_{\omega}^{\Lambda}(\mathbf{x})
$$

defines a measurable map

$$
\begin{aligned}
f: \Omega & \rightarrow \mathcal{C}^{0}(M, M), \\
\omega & \mapsto f_{\omega},
\end{aligned}
$$

whose qth coordinate function is given by (53).

Proof. - Fix $\omega \in \Omega, \mathbf{x} \in M$ and a metric $\varrho_{M}$ like in Definition 1.26. We show that $f_{\omega}$ is continuous in $\mathbf{x}$. For that let $\varepsilon>0$ and choose $R_{0} \in \mathbb{N}$ such that

$$
c_{S} b(q)<\varepsilon
$$

for all $q$ with $\|q\|>R_{0}$. We note that the $q$ th coordinate function of $\pi_{\Lambda_{1}} \circ f_{\omega} \in$ $\mathcal{C}^{0}\left(M,\left(S^{1}\right)^{\Lambda_{1}}\right)$ is the same as the $q$ th coordinate function $f_{\omega, q}$ of $f_{\omega}$.

By continuity of $\pi_{B_{R_{0}}(0)} \circ f_{\omega}$ we can choose a $\delta>0$ such that for all $\mathbf{y} \in B_{\delta}(\mathbf{x})$ and all $q$ with $\|q\| \leqslant R_{0}$ :

$$
c_{S} b(q) \varrho_{S^{1}}\left(f_{\omega, q}(\mathbf{x}), f_{\omega, q}(\mathbf{y})\right)<\varepsilon .
$$

From (55) and (56) we conclude that for all $\mathbf{y} \in B_{\delta}(\mathbf{x})$

$$
\varrho_{M}\left(f_{\omega}(\mathbf{x}), f_{\omega}(\mathbf{y})\right)<\varepsilon
$$

which was to be shown. Finally $f$ depends measurably on $\omega$ because it is pointwise limit of measurable functions with values in a metric space (cf. [22], p. 117, for example).

Remark 1.29. -

1. Lemma 1.28 is in particular based on the compactness on $M$ w.r.t. the product toplology.

$M$ is not compact w.r.t. the different metric, defined by

$$
\tilde{\varrho}_{M}(\mathbf{x}, \mathbf{y}) \stackrel{\text { def }}{=} \sup _{q \in \Lambda} \varrho_{S^{1}}\left(x_{q}, y_{q}\right) .
$$

In this case the conclusion from 'local' to 'global' does not hold.

2. As $f$ in (54) is $\left(\mathcal{A}, \mathcal{B}_{\mathcal{C}^{0}(M, M)}\right)$-measurable, the map $(\omega, \mathbf{x}) \mapsto f_{\omega}(\mathbf{x})$ is $(\mathcal{A} \times$ $\mathcal{B}_{M}, \mathcal{B}_{M}$ )-measurable. We have proved this fact in Example 1.18.

\section{Infinite-dimensional systems}

In Example 1.24 we used a counting process with values in $\mathbb{N}^{\Lambda}$ (for finite $\Lambda$ ) and a family of updating-maps on $Y^{\Lambda}$ to define Markov kernels on the product $Y^{\Lambda}$. These 
kernels act on the product space $\mathcal{C}^{0}\left(Y^{\Lambda}\right)$ of continuous functions (cf. Definition 1.19 and Proposition 2.15). In view of spatially extended systems like coupled map lattices or interacting particle systems we would like to define analogous operators for infinitedimensional systems $\left(\Lambda=\mathbb{Z}^{d}\right)$. As counting process we take the Poisson process $\left(\Omega, \mathcal{A}, P,\left(X_{t}\right)_{t \geqslant 0}\right)$ with parameter $\lambda>0$ and values in $\mathbb{N}^{d}$.

Recall that the set $\mathcal{N}_{1}$, defined in Definition 1.10, of all $\omega \in \Omega$ such that $X_{\text {. }}(\omega)$ is not nondecreasing, jumps at time 0 , has simultaneous jumps or jumps of size greater than one, has $P$-measure zero. So we have to consider updatings only at single sites. They are given by a family of continuous maps $\left(S_{q}\right)_{q \in \mathbb{Z}^{d}}$ such that $S_{q}: M \rightarrow M$ changes only the $q$ th coordinate (cf. Example 1.24 for a definition.)

A problem is obviously that the Poisson process, restricted to any finite interval $[0, t]$ of length $t>0$ is not of finite expectation (cf. Definition 1.1 and Remark 1.9.1). $P$-a.s. there are infinitely many jumps and it is even impossible to define an order preserving bijection between them and $\mathbb{N}$. However, in Section 2.1 we will show for systems with finite range interaction that for $P$-a.a. $\omega \in \Omega$, any $q \in \mathbb{Z}^{d}$ and $t>0$ the site $q$ is affected in $[0, t]$ (cf. Definition 2.1) by only finitely many sites, so that maps ' $\pi_{q} \circ S_{\omega}^{t}$, from $M$ to $\left(S^{1}\right)^{\{q\}}$ and then also ' $S_{\omega}^{t}$ ' from $M$ to $M$ can be defined in a natural way. The proof is based on a percolation argument. Percolation techniques, but different from the ones presented here, were already used by Harris in [15] for proving the existence of certain interacting particle systems of finite range. It follows in particular that $\pi_{\Lambda} \circ S_{\omega}^{t}: M \rightarrow\left(S^{1}\right)^{\Lambda}$ for finite $\Lambda \neq \emptyset$ is the limit (as $\tilde{\Lambda} \rightarrow \mathbb{Z}^{d}$ ) of maps that are constructed by using the 'cut offs' $\pi_{\Lambda} \circ S_{\tilde{\Lambda}, \xi, \omega}^{t}$, corresponding to a finite $\tilde{\Lambda} \supset \Lambda$ and boundary conditions $\xi$. In fact, this limit also exists and is independent of the boundary conditions for a huge class of infinite range interactions as we will show in Section 2.2. It gives rise to a natural definition of the system. But we also note that for infinite range interaction each site is with positive probability affected by infinitely many other sites. So we cannot use the same definition as for finite range interaction.

In Section 2.3 we define Markov kernels $K_{S}^{t}$ for the infinite system $S^{t}$ and $K_{S, \tilde{\Lambda}}^{t}$ for the system $S_{\tilde{\Lambda}}^{t}$ that fixes the $\tilde{\Lambda}^{C}$-coordinates for a finite $\tilde{\Lambda}$ (Recall the notation for the complement from Definition 1.11). We show that $K_{S}^{t}$ is the weak limit of $K_{S, \tilde{\Lambda}}^{t}$ (as $\tilde{\Lambda} \rightarrow \mathbb{Z}^{d}$ ), i.e. the corresponding operators on continuous functions converge weakly.

\subsection{Finite range interaction}

Now we are considering an interaction of range $R \in \mathbb{N} \backslash\{0\}$, i.e. $\pi_{q} \circ S_{q}(\mathbf{x})$ depends only on $\mathbf{x}_{B_{R}(q)}$. (Recall that $B_{R}(q)$ was defined in (23).)

DEFINITION 2.1. - Given $R$ as above, $q, \tilde{q} \in \mathbb{Z}^{d}, T>0, \omega \in \Omega$. We say that $\tilde{q}$ affects $q$ w.r.t. $(R, t, \omega)$ if there is a causal path from $q$ to $\tilde{q}$ of step size at most $R$. (Recall that we defined path etc. in Definitions 1.13 to 1.15$)$. If $\emptyset \neq \Lambda \subset \mathbb{Z}^{d}$ we say that $\tilde{q}$ affects $\Lambda$ w.r.t. $(R, t, \omega)$ if $\tilde{q}$ affects at least one point in $\Lambda$ w.r.t. $(R, t, \omega)$.

We set

$$
\begin{aligned}
& \operatorname{Aff}_{(R, t, \omega)}(\Lambda) \stackrel{\text { def }}{=}\left\{\tilde{q} \in \mathbb{Z}^{d}: \tilde{q} \text { affects } \Lambda \text { w.r.t. }(R, t, \omega)\right\} \\
& \quad \text { and } \Omega_{R} \stackrel{\text { def }}{=}\left\{\omega: \exists t>0, q \in \mathbb{Z}^{d} \text { such that }\left|\operatorname{Aff}_{(R, t, \omega)}(q)\right|=\infty\right\},
\end{aligned}
$$




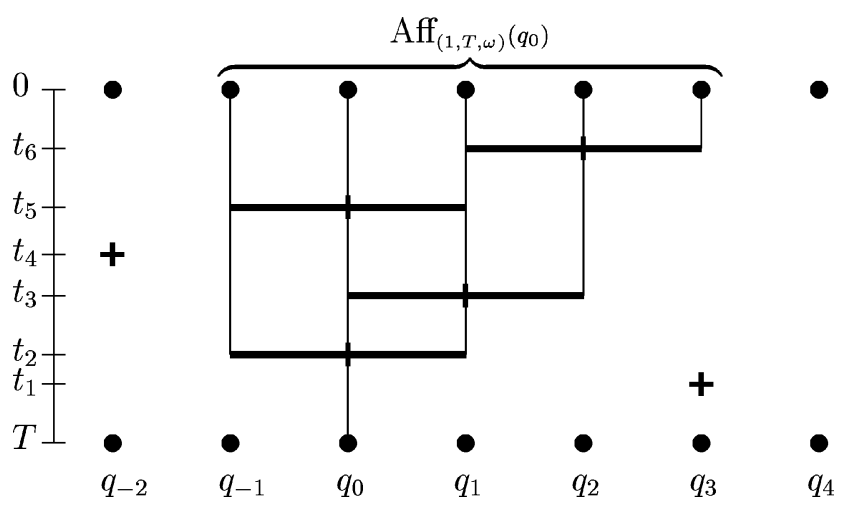

Fig. 1. The history of $q_{0}$.

where $|\cdot|$ denotes the cardinality.

Fig. 1 is a picture of $\operatorname{Aff}_{(1, T, \omega)}\left(q_{0}\right)$. We consider the finite time-interval $(0, T]$ and nearest neighbour interaction and a particular $\omega$. For each jump we draw a cross at the particular point $(q, t)$. There are jumps at $\left(q_{2}, t_{6}\right),\left(q_{0}, t_{5}\right),\left(q_{-2}, t_{4}\right),\left(q_{1}, t_{3}\right),\left(q_{0}, t_{2}\right)$ and $\left(q_{3}, t_{1}\right)$ The last jump at $q_{0}$ is at time $t_{2}$. We draw a thick horizontal line between $\left(q_{0}, t_{2}\right)$ and $\left(q, t_{2}\right)$ for all nearest neighbours $q$ of $q_{0}$ because the updating of $q_{2}$ depends also on these sites. So we have to consider the 'histories' of $q_{0}$ and its nearest neighbours before time $t_{2}$. Note that $q_{3} \in \operatorname{Aff}_{(1, T, \omega)}\left(q_{0}\right)$ and it is updated at time $t_{1}$ (and so affected by $q_{4}$ for example) but that updating has no influence on $q_{0}$ (at time $T$ ). We also note that, for example, $q_{-1}$ affects $q_{0}$ (w.r.t. $\left.(1, T, \omega)\right)$ but not the other way around. So we have to consider only the time- and space-ordered percolation.

Proposition 2.2. $-\Omega_{R}$ has P-measure zero:

$$
P\left(\Omega_{R}\right)=0 .
$$

Proof. $-\operatorname{Aff}_{(R, t, \omega)}(q)$ is increasing in $t$ and so

$$
\Omega_{R}=\bigcup_{t \in \mathbb{N}} \bigcup_{q \in \mathbb{Z}^{d}}\left\{\omega:\left|\operatorname{Aff}_{(R, t, \omega)}(q)\right|=\infty\right\} .
$$

So it is sufficient to show that for fixed $q \in \Lambda$ and $t>0$ the set $\left\{\omega:\left|\operatorname{Aff}_{(R, t, \omega)}(q)\right|=\infty\right\}$ has $P$-measure zero. If we set

$$
A_{N} \stackrel{\text { def }}{=}\left\{\omega: \operatorname{Aff}_{(R, t, \omega)}(q) \not \subset B_{N}(q)\right\}
$$

it is sufficient to show that

$$
\lim _{N \rightarrow \infty} P\left(A_{N}\right)=0 .
$$

If $q$ is affected by some $\tilde{q} \notin B_{N}(q)$ w.r.t. $(R, t, \omega)$ then there is a maximal causal path of step size at most $R$ from $q$ to $\tilde{q}$ with at least $N_{0}$ steps, where $N_{0}$ is the smallest integer greater than $\frac{N}{R}$. 
Consider any maximal causal path $Q=\left(q_{0}=q, \ldots, q_{n}\right)$ of step size at most $R$ and with $n \geqslant N_{0}$. $Q$ is a maximal causal path w.r.t. $(t, \omega)$ iff the trajectory of $\omega$ w.r.t. the Poisson process induced by $Q$ (cf. Definition 1.14) has exactly $n$ jumps. The probability of this is $p_{\lambda}(t, n)$ (which was defined in (4).)

We set

$$
c_{d, R} \stackrel{\text { def }}{=}\left|B_{R}(q)\right| .
$$

(Recall that $B_{R}(q)$ was defined in (23) and $|\cdot|$ denotes the cardinality.)

Then

$$
|\operatorname{Path}(q, n, R)|=c_{d, R}^{n}
$$

because at each step in the path one can choose between $c_{d, R}$ lattice-points.

So we have

$$
A_{N} \subset \bigcup_{n \geqslant N_{0}} \bigcup_{Q \in \operatorname{Path}(q, n, R)}\{\omega: Q \text { is maximal causal w.r.t. }(R, t, \omega)\}
$$

and so

$$
\begin{aligned}
P\left(A_{N}\right) & \leqslant \sum_{n \geqslant N_{0}} c_{d, R}^{n} \mathrm{e}^{-\lambda t} \frac{(\lambda t)^{n}}{n !} \\
& \leqslant \mathrm{e}^{\left(c_{d, R}-1\right) \lambda t}\left(c_{d, R} \lambda t\right)^{N_{0}} \frac{1}{N_{0} !}
\end{aligned}
$$

which converges to 0 as $N_{0} \rightarrow \infty$ which was to show. For the last inequality we have used the estimate for the Lagrange remainder in Taylor's formula.

DEFINITION 2.3. - Let a finite range interaction (i.e. a family of updatings) be given by $\left(S_{q}\right)_{q \in \mathbb{Z}^{d}}$. Fix $\omega \in \Omega \backslash\left(\Omega_{R} \cup \mathcal{N}_{1}\right), \emptyset \neq \Lambda \subset \tilde{\Lambda} \in \mathcal{F}, \xi \in M$ and $t>0$. Then $\omega$ has only finitely many jumps in $\tilde{\Lambda} \times(0, t)$, say at $\left(q_{1}, t_{1}\right), \ldots,\left(q_{n}, t_{n}\right)$ with $0<t_{1}<\cdots<t_{n}<t$.

We denote by $\mathbf{x}_{\tilde{\Lambda}} \vee \xi_{\tilde{\Lambda}^{c}}$ the point in $M$ that has the same $\tilde{\Lambda}$-coordinates as $\mathbf{x}$ and the same $\tilde{\Lambda}^{C}$-coordinates as $\xi$.

We define

$$
\begin{aligned}
& S_{q, \tilde{\Lambda}, \xi}:\left(S^{1}\right)^{\tilde{\Lambda}} \rightarrow\left(S^{1}\right)^{\tilde{\Lambda}}, \\
& S_{q, \tilde{\Lambda}, \xi}\left(\mathbf{x}_{\tilde{\Lambda}}\right) \stackrel{\text { def }}{=} \pi_{\tilde{\Lambda}} \circ S_{q}\left(\mathbf{x}_{\tilde{\Lambda}} \vee \xi_{\tilde{\Lambda}^{c}}\right),
\end{aligned}
$$

and

$$
\begin{aligned}
\Omega \backslash\left(\mathcal{N}_{1} \cup \Omega_{R}\right) \ni \omega & \mapsto S_{\tilde{\Lambda}, \xi, \omega}^{t} \in \mathcal{C}^{0}(M, M), \\
S_{\tilde{\Lambda}, \xi, \omega}^{t}(\mathbf{x}) & \stackrel{\text { def }}{=} S_{q_{n}, \tilde{\Lambda}, \xi} \circ \cdots \circ S_{q_{1}, \tilde{\Lambda}, \xi}\left(\mathbf{x}_{\tilde{\Lambda}}\right) \vee \xi_{\tilde{\Lambda}^{c}} .
\end{aligned}
$$

The maps $S_{\tilde{\Lambda}, \xi, \omega}^{t}$ are continuous as composites of continuous maps. Furthermore, $S_{\tilde{\Lambda}, \xi, \omega}^{t}$ depends only on $\omega_{\tilde{\Lambda}}$ (i.e. on $\left.\pi_{\tilde{\Lambda}} \circ X .(\omega)\right)$ and (70) gives rise to a countable, measurable partition of $\Omega \backslash\left(\mathcal{N}_{1} \cup \Omega_{R}\right): \omega$ and $\tilde{\omega}$ belong to the same set of this partition if they have the same list of jump sites $\left(q_{1}, \ldots, q_{n}\right)$ (ordered w.r.t. the jump times).

Now let $\tilde{\Lambda} \supset \operatorname{Aff}_{(R, t, \omega)}(\Lambda)$ and $\xi \in M$ and define 


$$
\begin{aligned}
& \pi_{\Lambda} \circ S^{t}: \Omega \backslash\left(\mathcal{N}_{1} \cup \Omega_{R}\right) \rightarrow \mathcal{C}^{0}\left(M,\left(S^{1}\right)^{\Lambda}\right), \\
& \pi_{\Lambda} \circ S_{\omega}^{t}(\mathbf{x}) \stackrel{\text { def }}{=} \pi_{\Lambda} \circ S_{\tilde{\Lambda}, \xi, \omega}^{t}\left(\mathbf{x}_{\tilde{\Lambda}}\right) .
\end{aligned}
$$

The definition does not depend on the choice of $\tilde{\Lambda}$ or $\xi$ because the right-hand side (rhs) of (72) depends, by definition, on the $\operatorname{Aff}_{(R, t, \omega)}(\Lambda)$-coordinates of $\mathbf{x}$ only.

Further, the family $\left(\pi_{\Lambda} \circ S_{\omega}^{t}(\mathbf{x})\right)_{\Lambda \in \mathcal{F} \backslash\{\emptyset\}}$ is consistent in the sense that for any $\emptyset \neq$ $\Lambda_{1} \subset \Lambda_{2} \in \mathcal{F}$ :

$$
\pi_{\Lambda_{1}}\left(\pi_{\Lambda_{2}} \circ S_{\omega}^{t}(x)\right)=\pi_{\Lambda_{1}} \circ S_{\omega}^{t}(x)
$$

and so defines a map

$$
\begin{gathered}
S_{\omega}^{t}: M \rightarrow M, \\
\left(S_{\omega}^{t}(x)\right)_{q} \stackrel{\text { def }}{=} \pi_{q} \circ S_{\omega}^{t}(x) .
\end{gathered}
$$

Finally, we set $S_{\omega}^{t}=\operatorname{id}_{M}$ for $\omega \in \Omega_{R} \cup \mathcal{N}$.

Proposition 2.4. - The map $S_{\omega}^{t}$, defined in (72) and (74) is continuous and depends measurably on $\omega$.

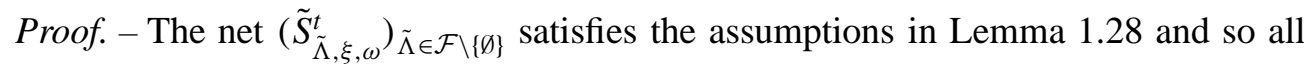
statements of Proposition 2.4 follow.

\subsection{Infinite range interaction}

We extend our notion of ' $S_{\omega}^{t}$ ' to interactions that are not necessarily of finite range.

Consider a family $\left(S_{q}\right)_{q \in \mathbb{Z}^{d}}$ of maps $S_{q}: M \rightarrow M$ such that $S_{q}$ does not change the $\mathbb{Z}^{d} \backslash\{q\}$-coordinates and $\pi_{q} \circ S_{q}: M \rightarrow S^{1}$ is Lipschitz-continuous w.r.t. all coordinates and the Lipschitz constants depend only on the relative positions of the sites, i.e. there are constants $w(r)$ for all $r \in \mathbb{Z}^{d}$ such that for all $q, \tilde{q} \in \mathbb{Z}^{d}$ and $\mathbf{x}, \mathbf{y} \in M$ with $\mathbf{x}_{\mathbb{Z}^{d} \backslash\{\tilde{q}\}}=\mathbf{y}_{\mathbb{Z}^{d} \backslash\{\tilde{q}\}}$ (i.e. $\mathbf{x}$ and $\mathbf{y}$ differ at most in their $\tilde{q}$-coordinates.)

$$
\varrho_{S^{1}}\left(\pi_{q} \circ S_{q}(\mathbf{x}), \pi_{q} \circ S_{q}(\mathbf{y})\right) \leqslant w(\tilde{q}-q) \varrho_{S^{1}}\left(y_{\tilde{q}}, z_{\tilde{q}}\right) .
$$

We further assume summability of the Lipschitz-constants, i.e.

$$
\sum_{q \in \mathbb{Z}^{d}} w(q)=c_{1}
$$

with a positive constant $c_{1}$.

We need the following technical lemma.

LEMMA 2.5. - If $(w(q))_{q \in \mathbb{Z}^{d}}$ is a family of non-negative real numbers satisfying (76) then there are families $\left(w_{1}(q)\right)_{q \in \mathbb{Z}^{d}}$ and $\left(w_{2}(q)\right)_{q \in \mathbb{Z}^{d}}$ of non-negative and positive numbers, respectively, such that

$$
\begin{aligned}
w(q) & =w_{1}(q) w_{2}(q) \quad \text { for all } q \in \mathbb{Z}^{d}, \\
\sum_{q \in \mathbb{Z}^{d}} w_{1}(q) & \leqslant 2 c_{1}+1, \\
\text { and } \lim _{R \rightarrow \infty} a(R) & =0,
\end{aligned}
$$


where the positive function a $(\cdot)$ is defined by

$$
a(R) \stackrel{\text { def }}{=} \sup _{\left\|r_{1}\right\|+\cdots+\left\|r_{n}\right\|=R} w_{2}\left(r_{1}\right) \cdots \cdots w_{2}\left(r_{n}\right) .
$$

(The empty product is defined to be equal to 1.$)$

Proof. - We can choose $r_{0}=0<r_{1}<\cdots \in \mathbb{N}$ such that

$$
\sum_{\|q\|<r_{i}} w(q) \geqslant c_{1}-4^{-(i+1)} \quad \text { for } i \geqslant 1 .
$$

Then we have

$$
\sum_{\|q\|<r_{1}} w(q) \leqslant c_{1} \quad \text { and } \quad \sum_{r_{i} \leqslant\|q\|<r_{i+1}} w(q) \leqslant 4^{-(i+1)} \quad \text { for } i \geqslant 1 .
$$

We set for $i \geqslant 1$ and $r_{i-1} \leqslant\|q\|<r_{i}$ :

$$
\begin{aligned}
& w_{2}(q) \stackrel{\text { def }}{=} 2^{-i}, \\
& w_{1}(q) \stackrel{\text { def }}{=} 2^{i} w(q) .
\end{aligned}
$$

Then (77) is obviously satisfied. To prove (78) we use (82) and (84):

$$
\sum_{q \in \mathbb{Z}^{d}} w_{1}(q)=\sum_{i=0}^{\infty} \sum_{r_{i} \leqslant\|q\|<r_{i+1}} w_{1}(q) \leqslant 2 c_{1}+\sum_{i=1}^{\infty} 2^{-i}=2 c_{1}+1 .
$$

Now we prove (79). We show by induction (w.r.t. $i$ ) that for every $i \geqslant 1$ there is an $n_{i}$ such that

$$
a(R)<2^{-i} \text { for all } R \geqslant n_{i} .
$$

For $i=1$ the statement is true with $n_{1}=1$ because $a(R) \leqslant \frac{1}{2}$ for every $R \geqslant 1$ as there is at least one factor on the right-hand-side in (80) and each such factor is at most $\frac{1}{2}$.

Now we assume that the statement holds for $i$ and $n_{i}$. We set

$$
n_{i+1} \stackrel{\text { def }}{=} r_{i}+2 n_{i} .
$$

Then every path $\left(q_{0}, \ldots, q_{n}\right)$ of length $R \geqslant n_{i+1}$ has at least one step of size at least $r_{i}$ (i.e. there is an $1 \leqslant l \leqslant n$ such that $\left\|q_{l}-q_{l-1}\right\| \geqslant r_{i}$ ) or it can be divided into two paths both of length at least $n_{i}$ (i.e. there is an $1 \leqslant l \leqslant m-1$ such that $\left\|q_{0}-q_{1}\right\|+\cdots+\left\|q_{l-1}+q_{l}\right\| \geqslant n_{i}$ and $\left.\left\|q_{l+1}-q_{l}\right\|+\cdots+\left\|q_{n}+q_{n-1}\right\| \geqslant n_{i}\right)$. So each product on the right-hand side of (80) has at least one factor less than or equal to $2^{-(i+1)}$ or two factors less than or equal to $2^{-i}$. As the other factors are smaller than 1 the product is bounded by $2^{-(i+1)}$ as was to be shown.

Now we fix (like in Lemma 2.5) a choice of $\left(w_{1}(q)\right)_{q \in \mathbb{Z}^{d}}$ and $\left(w_{2}(q)\right)_{q \in \mathbb{Z}^{d}}$ and so the function $a$. 
DEFINITION 2.6. - We fix the metric $\varrho_{M}$ on $M$ by

$$
\varrho_{M}(\mathbf{x}, \mathbf{y}) \stackrel{\text { def }}{=} \sup _{r \in \mathbb{Z}^{d}} a(\|r\|) \varrho_{S^{1}}\left(x_{r}, y_{r}\right)
$$

Remark 2.7. - It follows from Remark 1.27.1 and (79) that $\varrho_{M}$ is a metric and compatible with the product topology.

LEMMA 2.8. - The maps $S_{q}: M \rightarrow M$ are continuous (w.r.t. the product topology on $M)$.

Proof. - According to Remark 1.27.2 and the uniform choice of the Lipschitzconstants (cf. (75)) we only have to show that the maps $\pi_{q} \circ S_{0}: M \rightarrow S^{1}$ are continuous.

If $q \neq 0$ then the $q$ th coordinate is not changed by $S_{0}$ and

$$
a(\|q\|) \varrho_{S^{1}}\left(\pi_{q} \circ S_{0}(\mathbf{x}), \pi_{q} \circ S_{0}(\mathbf{y})\right)=a(\|q\|) \varrho_{S^{1}}\left(x_{q}, y_{q}\right) \leqslant \varrho_{M}(\mathbf{x}, \mathbf{y}) .
$$

If $q=0$ we estimate

$$
\begin{aligned}
a(0) \varrho_{S^{1}}\left(\pi_{0} \circ S_{0}(\mathbf{x}), \pi_{0} \circ S_{0}(\mathbf{y})\right) & \leqslant a(0) \sum_{r \in \mathbb{Z}^{d}} w(r) \varrho_{S^{1}}\left(x_{r}, y_{r}\right) \\
& \leqslant a(0) \sum_{r \in \mathbb{Z}^{d}} w(r) \frac{1}{a(\|r\|)} \varrho_{M}(\mathbf{x}, \mathbf{y}) \\
& \leqslant a(0)\left(2 c_{1}+1\right) \varrho_{M}(\mathbf{x}, \mathbf{y}),
\end{aligned}
$$

where we have used (75) for the first, the definition of $\varrho_{M}$ for the second and (77) for the third inequality. So $\pi_{q} \circ S_{0}$ is continuous for all $q \in \mathbb{Z}^{d}$.

In the following we estimate the distance (w.r.t. the uniform norm) between $\pi_{0} \circ S_{\Lambda, \xi, \omega}^{t}$ and $\pi_{0} \circ S_{\Lambda, \tilde{\xi}, \omega}^{t}$ for different boundary conditions $\xi_{\Lambda^{c}}$ and $\tilde{\xi}_{\Lambda^{c}}$ (that might even depend on the time) at the $\Lambda^{C}$-sites. Conditions (75) and (76) allow us to apply standard estimates for the 'error-growth' for composites of maps. Using the linear nature of the 'Lipschitzcondition' (75), we write the products of sums (over all coordinates, like in (75)) as sums (over paths) of products (corresponding to the particular paths).

We fix $t>0, \Lambda \in \mathcal{F}$ and $\omega \in \Omega \backslash \mathcal{N}_{1}$. By definition of $\mathcal{N}_{1}$ (cf. (21)) $\omega$ has no jumps at 0 , no simultaneous jumps and only finitely many jumps in $\Lambda \times(0, t)$, say at $\left(q_{1}, t_{1}\right), \ldots,\left(q_{N}, t_{N}\right)$ with $0<t_{1}<\cdots<t_{N}<t$. We set $t_{0} \stackrel{\text { def }}{=} 0$ and fix arbitrary $\xi=\left(\xi\left(t_{0}\right), \ldots, \xi\left(t_{N}\right)\right), \tilde{\xi}=\left(\tilde{\xi}\left(t_{0}\right), \ldots, \tilde{\xi}\left(t_{N}\right)\right) \in M^{N+1}$ and $\mathbf{x}, \mathbf{y} \in M$.

We set $\mathbf{x}(0) \stackrel{\text { def }}{=} \mathbf{x}_{\Lambda} \vee \xi_{\Lambda^{c}}(0), \mathbf{y}(0) \stackrel{\text { def }}{=} \mathbf{y}_{\Lambda} \vee \tilde{\xi}_{\Lambda^{c}}(0)$ and define for $1 \leqslant i \leqslant N$ recursively:

$$
x_{q}\left(t_{i}\right) \stackrel{\text { def }}{=} \begin{cases}\pi_{q} \circ S_{q}\left(\mathbf{x}\left(t_{i-1}\right)\right) & \text { for } q=q_{i}, \\ x_{q}\left(t_{i-1}\right) & \text { for } q \in \Lambda \backslash\left\{q_{i}\right\}, \\ \xi_{q}\left(t_{i}\right) & \text { for } q \in \Lambda^{C} .\end{cases}
$$

We define $\mathbf{y}\left(t_{i}\right)$ analogously, using $\mathbf{y}$ and $\tilde{\xi}$ instead of $\mathbf{x}$ and $\xi$, respectively. 
Two points in $S^{1}$ can have distance at most $c_{S}=\operatorname{diam}_{\varrho_{S}}\left(S^{1}\right)$. For estimating the distance between $x_{q}\left(t_{i}\right)$ and $y_{q}\left(t_{i}\right)$ we define

$$
\Delta_{q}(0) \stackrel{\text { def }}{=} \tilde{\Delta}_{q}(0) \stackrel{\text { def }}{=} \begin{cases}\varrho_{S^{1}}\left(x_{q}(0), y_{q}(0)\right) & \text { for } q \in \Lambda, \\ c_{S} & \text { for } q \in \Lambda^{C},\end{cases}
$$

and for $1 \leqslant i \leqslant N$

$$
\begin{aligned}
& \Delta_{q}(i) \stackrel{\text { def }}{=} \begin{cases}\sum_{r \in \mathbb{Z}^{d}} w(r-q) \Delta_{r}(i-1) & \text { for } q=q_{i}, \\
\Delta_{q}(i-1) & \text { for } q \in \Lambda \backslash\left\{q_{i}\right\}, \\
c_{S} & \text { for } q \in \Lambda^{C},\end{cases} \\
& \tilde{\Delta}_{q}(i) \stackrel{\text { def }}{=} \begin{cases}\min \left\{c_{S}, \sum_{r \in \mathbb{Z}^{d}} w(r-q) \tilde{\Delta}_{r}(i-1)\right\} & \text { for } q=q_{i}, \\
\tilde{\Delta}_{q}(i-1) & \text { for } q \in \Lambda \backslash\left\{q_{i}\right\}, \\
c_{S} & \text { for } q \in \Lambda^{C} .\end{cases}
\end{aligned}
$$

The functions $\Delta_{q}$ and $\tilde{\Delta}_{q}$ depend on $\mathbf{x}, \mathbf{y}$ and $\Lambda$ but we do not refer to this in our notation. We have introduced them for estimating the difference between $x_{q}\left(t_{i}\right)$ and $y_{q}\left(t_{i}\right)$ (cf. (94)) and so the difference between $x_{q}(t)$ and $y_{q}(t)$. This difference depends also on $\omega$ and so do the corresponding estimates for $\Delta_{q}$ and $\tilde{\Delta}_{q}$. In Definition 2.11 we will relate $\Delta_{q}$ and $\tilde{\Delta}_{q}$ to families of random variables $\left(Y_{\Lambda}\right)_{\Lambda \in \mathcal{F} \backslash\{\emptyset\}}$ and $\left(\tilde{Y}_{\Lambda}\right)_{\Lambda \in \mathcal{F} \backslash\{\emptyset\}}$, respectively. For $\Delta_{q}$ we find a particularly nice expansion (cf. (95)). From this follows the convergence of $Y_{\Lambda}$ to zero in expectation (as $\Lambda \rightarrow \mathbb{Z}^{d}$ ). We will show that $\tilde{Y}_{\Lambda}$ is bounded by $Y_{\Lambda}$ and decreasing and so converges $P$-almost surely to zero by the Monotone Convergence Theorem (cf. Theorem 2.13).

Proposition 2.9. - The following holds for $0 \leqslant i \leqslant N$ :

1 .

$$
\varrho_{S^{1}}\left(x_{q}\left(t_{i}\right), y_{q}\left(t_{i}\right)\right) \leqslant \tilde{\Delta}_{q}(i) \leqslant \Delta_{q}(i)
$$

2.

$$
\Delta_{q}(i)=\sum_{\substack{\left(r_{0}=q, r_{1}, \ldots, r_{n}\right) \\ \in \operatorname{Path}_{\mathbf{C}}\left(t_{i}, \omega, q, \Lambda\right)}} w\left(r_{1}-r_{0}\right) \cdots w\left(r_{n}-r_{n-1}\right) \Delta_{r_{n}}(0) .
$$

3. If in particular $\mathbf{x}_{\Lambda}=\mathbf{y}_{\Lambda}$ and $q \in \Lambda$ then

$$
\Delta_{q}(N(\omega)) \leqslant c_{S} a\left(\operatorname{dist}_{\mathbb{Z}^{d}}\left(q, \Lambda^{C}\right)\right) \sum_{\substack{\left(r_{0}=q, r_{1}, \ldots, r_{n}\right) \\ \in \operatorname{Path}_{\mathcal{C}}\left(t, \omega, q \rightarrow \Lambda^{C}\right)}} w_{1}\left(r_{1}-r_{0}\right) \cdots w_{1}\left(r_{n}-r_{n-1}\right),
$$

where $N(\omega)$ is the number of jumps of $\omega$ in $\Lambda \times(0, t)$.

Proof. - We prove (94) and (95) by induction w.r.t. $i$.

$i=0$ : (94) holds by definition of $\Delta_{q}(0)$ and $\tilde{\Delta}_{q}(0)$ (cf. (92)). At time 0 no jump has happened and the only summand on the right-hand-side in (95) corresponds to the empty path at site $q$ and so the equality in (95) holds.

$i-1 \rightarrow i$ : (95) holds obviously for $i$ and $q \neq q_{i}$ as there is no updating at site $q$ and

$$
\operatorname{Path}_{\mathrm{c}}\left(t_{i}, \omega, q, \Lambda\right)=\operatorname{Path}_{\mathrm{c}}\left(t_{i-1}, \omega, q, \Lambda\right) .
$$


At site $q_{i}$ there is a jump at time $t_{i}$ and so we have

$$
\Delta_{q_{i}}(i)=\sum_{r \in \mathbb{Z}^{d}} w\left(r-q_{i}\right) \Delta_{r}(i-1) .
$$

Using the representation (95) for $\Delta_{r}(i-1)$ and the fact that every $\left(q_{i}, r_{1}, \ldots, r_{n}\right) \in$ Path $_{\mathcal{C}}\left(t_{i}, \omega, q_{i}, \Lambda\right)$ can be (uniquely) split into $\left(q_{i}, r_{1}\right)$ and $\left(r_{1}, \ldots, r_{n}\right) \in \operatorname{Path}_{\mathcal{C}}\left(t_{i-1}, \omega\right.$, $r_{1}, \Lambda$ ), we see that (95) holds for $i$.

Next we show the first inequality in (94) for $i$. For $q \in \Lambda^{C}$ the distances between $x_{q}\left(t_{i}\right)=\xi_{q}\left(t_{i}\right)$ and $y_{q}\left(t_{i}\right)=\tilde{\xi}_{q}\left(t_{i}\right)$ is bounded by $c_{S}$ and for $q \in \Lambda \backslash\left\{q_{i}\right\}$ we have $x_{q}\left(t_{i}\right)=x_{q}\left(t_{i-1}\right)$ and $y_{q}\left(t_{i}\right)=y_{q}\left(t_{i-1}\right)$. So in both cases the first inequality in (94) holds.

Now we consider the site $q_{i}$ where a jump happens at time $t_{i}$. Using (75), assumption (94), for $i-1$, and (98), we get

$$
\begin{aligned}
\varrho_{S^{1}}\left(x_{q}\left(t_{i}\right), y_{q}\left(t_{i}\right)\right) & \leqslant \sum_{r \in \mathbb{Z}^{d}} w\left(r-q_{i}\right) \varrho_{S^{1}}\left(x_{r}\left(t_{i-1}\right), y_{r}\left(t_{i-1}\right)\right) \\
& \leqslant \sum_{r \in \mathbb{Z}^{d}} w\left(r-q_{i}\right) \Delta_{r}(i-1) \leqslant \Delta_{q_{i}}(i) .
\end{aligned}
$$

So the first inequality in (94) is proved for $i$. The second follows immediately from (94). So statements 1 and 2 are proved.

Finally, (96) follows from (95): $\Delta_{q}(0)=0$ for $q \in \Lambda$. So we only have to sum over paths $\left(r_{0}=q, \ldots, r_{n}\right)$ that end in $r_{n} \in \Lambda^{C}$.

In particular, if we set $R \stackrel{\text { def }}{=}\left\|r_{n}\right\|$, then

$$
\begin{aligned}
\tilde{\Delta}_{r_{n}}(0) & =c_{S}, \\
\operatorname{dist}_{\mathbb{Z}^{d}}\left(q, \Lambda^{C}\right) & \leqslant R, \\
R & \leqslant\left\|r_{n}-r_{n-1}\right\|+\cdots+\left\|r_{1}-r_{0}\right\|,
\end{aligned}
$$

and so by the choice of $w_{1}, w_{2}$ and $a$, made before Definition 2.6, we get

$$
\begin{aligned}
& w\left(r_{1}-r_{0}\right) \cdots w\left(r_{n}-r_{n-1}\right) \\
& \quad \leqslant w_{1}\left(r_{1}-r_{0}\right) \cdots \cdots w_{1}\left(r_{n}-r_{n-1}\right) a(R) \\
& \quad \leqslant w_{1}\left(r_{1}-r_{0}\right) \cdots \cdots w_{1}\left(r_{n}-r_{n-1}\right) a\left(\operatorname{dist}_{\mathbb{Z}^{d}}\left(q, \Lambda^{C}\right)\right) .
\end{aligned}
$$

Using (95), (100) and (103), we get (96) .

Remark 2.10. - The summing over causal paths in Proposition 2.9 reflects that the result of an updating depends only on what has happened before.

Definition 2.11. - We define two families $\left(Y_{\Lambda}\right)_{\Lambda \in \mathcal{F} \backslash\{\emptyset\}}$ and $\left(\tilde{Y}_{\Lambda}\right)_{\Lambda \in \mathcal{F} \backslash\{\emptyset\}}$ of random variables on $\Omega \backslash \mathcal{N}_{1}$. Let $\Lambda \in \mathcal{F} \backslash\{\emptyset\}$ and $\omega \in \Omega \backslash \mathcal{N}_{1}$, say with exactly $N(\omega)$ jumps in $\Lambda \times[0, t]$. If we choose $\mathbf{x}, \mathbf{y} \in M$ with $\mathbf{x}_{\Lambda}=\mathbf{y}_{\Lambda}$ the value of $\Delta_{0}(N(\omega)$ ) (as defined by (92) and (94)) does not depend on $\mathbf{x}$ or $\mathbf{y}$. We define $Y_{\Lambda}(\omega)$ to be equal to this value:

$$
Y_{\Lambda}(\omega) \stackrel{\text { def }}{=} \Delta_{0}(N(\omega))
$$

$\tilde{Y}_{\Lambda}$ is defined analogously, using $\tilde{\Delta}_{0}(N(\omega))$ instead of $\Delta_{0}(N(\omega))$. 


\section{Remark 2.12.-}

1. We remark that $Y_{\Lambda}$ depends measurably on $\omega$. In fact there is a countable, measurable partition of $\Omega \backslash \mathcal{N}_{1}$ such that $\omega$ and $\tilde{\omega}$ belong to the same set (of that partition) if the sums for $\Delta_{0}\left(t_{N(\omega)}\right)$ and $\Delta_{0}\left(t_{N(\tilde{\omega})}\right)$ (cf. (95)) are over the same paths.

2. From (94) we see that

$$
\tilde{Y}_{\Lambda} \leqslant Y_{\Lambda}
$$

Now we fix $\xi, \mathbf{x} \in M$ and define the map $S_{\Lambda, \xi, \omega}^{t}$ like in (70).

THEOREM 2.13.-

1. There is a set $\mathcal{N}_{2}$ of $P$-measure zero such that

$$
\lim _{\Lambda \rightarrow Z^{d}} \tilde{Y}_{\Lambda}=0 \quad \text { for } \omega \in \Omega \backslash\left(\mathcal{N}_{1} \cup \mathcal{N}_{2}\right) .
$$

2. The limit

$$
\pi_{0} \circ S_{\omega}^{t} \stackrel{\text { def }}{=} \lim _{\Lambda \rightarrow \mathbb{Z}^{d}} \pi_{0} \circ S_{\Lambda, \xi, \omega}^{t}
$$

exists in $\mathcal{C}^{0}\left(M, S^{1}\right)$ for all $\omega \in \Omega \backslash\left(\mathcal{N}_{1} \cup \mathcal{N}_{2}\right)$. It is measurable in $\omega$ and does not depend on $\xi$.

3. There is a set $\mathcal{N} \subset \Omega$ of $P$-measure zero such that we can define maps

$$
\pi_{q} \circ S_{\omega}^{t} \stackrel{\text { def }}{=} \lim _{q \in \Lambda \rightarrow \mathbb{Z}^{d}} \pi_{q} \circ S_{\Lambda, \xi, \omega}^{t}
$$

for all $q \in \mathbb{Z}^{d}$ and $\omega \in \Omega \backslash \mathcal{N}$.

Further, we can define a map $S_{\omega}^{t} \in \mathcal{C}^{0}(M, M)$ by

$$
\left(S_{\omega}^{t}(\mathbf{x})\right)_{q} \stackrel{\text { def }}{=} \pi_{q} \circ S_{\omega}^{t}(\mathbf{x}) .
$$

$S_{\omega}^{t}$ depends measurably on $\omega$.

Proof. - First we show that

$$
\lim _{\Lambda \rightarrow \mathbb{Z}^{d}} E\left(Y_{\Lambda}\right)=0
$$

We set $R \stackrel{\text { def }}{=} \operatorname{dist}_{\mathbb{Z}^{d}}\left(0, \Lambda^{C}\right)$. Using (96) we get

$$
\begin{aligned}
E\left(Y_{\Lambda}\right) \leqslant & \int_{\Omega} \mathrm{d} P(\omega) c_{S} a(R) \sum_{\begin{array}{c}
\left(r_{0}=0, r_{1}, \ldots, r_{n}\right) \\
\in \operatorname{Path}_{\mathcal{C}}\left(t, \omega, 0 \rightarrow \Lambda^{C}\right)
\end{array}} w_{1}\left(r_{1}-r_{0}\right) \cdots w_{1}\left(r_{n}-r_{n-1}\right) \\
= & c_{S} a(R) \sum_{Q \in \operatorname{Path}_{\left(0 \rightarrow \Lambda^{C}\right)}} w_{1}\left(r_{1}-r_{0}\right) \cdots w_{1}\left(r_{n}-r_{n-1}\right) \\
& \times P\left(\left\{\omega: Q \in \operatorname{Path}_{\mathcal{C}}\left(t, \omega, 0 \rightarrow \Lambda^{C}\right)\right\}\right)
\end{aligned}
$$

A path $Q=\left(q_{0}=0, q_{1}, \ldots, q_{n}\right)$ with $q_{n} \in \Lambda^{C}$ is causal w.r.t. $(t, \omega)$ (i.e. $Q \in$ $\left.\operatorname{Path}_{\mathrm{C}}\left(t, \omega, 0 \rightarrow \Lambda^{C}\right)\right)$ iff the Poisson process induced by $Q$ has at least $n$ jumps. So we can estimate the probability 


$$
\begin{aligned}
P\left(\left\{\omega: Q \in \operatorname{Path}_{\mathrm{c}}\left(t, \omega, 0 \rightarrow \Lambda^{C}\right)\right\}\right) & =\sum_{m \geqslant n} \mathrm{e}^{-\lambda t} \frac{(\lambda t)^{m}}{m !} \\
& \leqslant \frac{(\lambda t)^{n}}{n !} .
\end{aligned}
$$

For the last line we have used Taylor's formula, as we did in (68). So we get, using (78),

$$
\begin{aligned}
E\left(Y_{\Lambda}\right) & \leqslant c_{S} a(R) \sum_{n=1}^{\infty} \frac{(\lambda t)^{n}}{n !}\left(\sum_{r \in \mathbb{Z}^{d}} w_{1}(r)\right)^{n} \\
& \leqslant c_{2} a(R)
\end{aligned}
$$

with $c_{2}=c_{S} \mathrm{e}^{\lambda t\left(2 c_{1}+1\right)}$. (Recall that we consider a fixed $t$ at the moment, so $c_{2}$ is a constant.) By (79) we get

$$
\lim _{\Lambda \rightarrow Z^{d}} E\left(Y_{\Lambda}\right)=0
$$

and, using (105),

$$
\lim _{\Lambda \rightarrow Z^{d}} E\left(\tilde{Y}_{\Lambda}\right)=0
$$

$\tilde{Y}_{\Lambda}(\omega)$ is decreasing for all $\omega \in \Omega \backslash \mathcal{N}_{1}$ : For a fixed $\omega$ and $0 \in \Lambda_{1} \subset \Lambda_{2} \in \mathcal{F}$ we consider the (time-ordered) sequence of jumps $\left(q_{1}, t_{1}\right), \ldots,\left(q_{n}, t_{n}\right)$ of $\omega$ at sites $q_{1}, \ldots, q_{2} \in$ $\Lambda_{1}$. It is a subsequence of the sequence of jumps $\left(\bar{q}_{1}, \bar{t}_{1}\right), \ldots,\left(\bar{q}_{m}, \bar{t}_{m}\right)$ of $\omega$ at sites $\bar{q}_{1}, \ldots, \bar{q}_{m} \in \Lambda_{2}$. The jumps $\left(q_{i}, t_{i}\right)$ in the first sequence correspond to jumps $\left(\bar{q}_{j(i)}, \bar{t}_{j(i)}\right)$ in the second one. Then $q_{i}=\bar{q}_{j(i)}$ and $t_{i}=\bar{t}_{j(i)}$ but the indices $i$ and $j(i)$ are not the same in general.

We define $\tilde{\Delta}_{q}^{1}(i)$ and $\tilde{\Delta}_{q}^{2}(j)$ as in (92) and (94) for the sets $\Lambda_{1}$ and $\Lambda_{2}$, respectively. We show that

$$
\tilde{\Delta}_{q}^{1}(i) \geqslant \tilde{\Delta}_{q}^{2}(j(i)) .
$$

If $q \in \Lambda_{1}^{C}$ then (118) obviously holds because $\tilde{\Delta}_{q}^{1}(i)=c_{S}$ is an upper bound for $\tilde{\Delta}_{q}^{2}(j)$. For $q \in \Lambda_{1}$ we show (118) by induction w.r.t. $i$.

If $i=0$ then (118) is true by (92). Now assume that (118) holds for all $q$ and a particular $i<n$. For $q \in \Lambda_{1} \backslash\left\{q_{i+1}\right\}$ we have

$$
\tilde{\Delta}_{q}^{1}(i+1)=\tilde{\Delta}_{q}^{1}(i) \geqslant \tilde{\Delta}_{q}^{2}(j(i))=\tilde{\Delta}_{q}^{2}(j(i+1))
$$

where the inequality holds by assumption and the equalities by (94). For the site $q=q_{i+1}$ we have by (94)

$$
\begin{aligned}
\tilde{\Delta}_{q}^{1}(i+1) & =\max \left\{c_{S}, \sum_{r \in \mathbb{Z}^{d}} w(r-q) \tilde{\Delta}_{r}^{1}(i)\right\} \\
& \geqslant \max \left\{c_{S}, \sum_{r \in \mathbb{Z}^{d}} w(r-q) \tilde{\Delta}_{r}^{2}(j(i+1)-1)\right\} \\
& =\tilde{\Delta}_{q}^{2}(j(i+1))
\end{aligned}
$$


which was to be shown. Here we have used that $\tilde{\Delta}_{r}^{1}(i) \geqslant \tilde{\Delta}_{r}^{2}(j(i+1)-1)$. This follows for $r \in \Lambda_{1}^{C}$ from the definition of $\tilde{\Delta}_{r}^{1}$ and $\tilde{\Delta}_{r}^{2}$ and for $r \in \Lambda_{1}$ from assumption (118) and the fact that $\tilde{\Delta}_{r}^{2}(j(i+1)-1)=\tilde{\Delta}_{r}^{2}(j(i))$.

Using the definition of $\tilde{Y}_{\Lambda_{1}}(\omega)$ and $\tilde{Y}_{\Lambda_{2}}(\omega)$ (cf. Definition 2.11), we conclude

$$
\tilde{Y}_{\Lambda_{1}}(\omega) \geqslant \tilde{Y}_{\Lambda_{2}}(\omega)
$$

which was to be shown.

We have proved (117) and that $\left(\tilde{Y}_{\Lambda}\right)_{\Lambda \in \mathcal{F} \backslash\{\emptyset\}}$ is decreasing. So we conclude (106), by using the Monotone Convergence Theorem.

Now we prove the second statement in Theorem 2.13, using the first one. First we note that for $\omega \in \Omega \backslash\left(\mathcal{N}_{1} \cup \mathcal{N}_{2}\right)$ the map $S_{\Lambda, \xi, \omega}^{t}$ is continuous since it is the composite of finitely many continuous (cf. Lemma 2.8) updating maps.

For $\Lambda \subset \tilde{\Lambda}$ we have

$$
\varrho_{\mathcal{C}^{0}\left(M, S^{1}\right)}\left(\pi_{0} \circ S_{\Lambda, \xi, \omega}^{t}, \pi_{0} \circ S_{\tilde{\Lambda}, \xi, \omega}^{t}\right) \leqslant \tilde{Y}_{\Lambda}(\omega) .
$$

So by (106) the net $\left(\pi_{0} \circ S_{\Lambda, \xi, \omega}^{t}\right)_{\Lambda \in \mathcal{F} \backslash\{\emptyset\}}$ is a Cauchy net with values in $\mathcal{C}^{0}\left(M, S^{1}\right)$ for $\omega \in \Omega \backslash\left(\mathcal{N}_{1} \cup \mathcal{N}_{2}\right)$ and so converges. Furthermore, it is a pointwise limit, i.e. for each particular $\omega$, and so $\pi_{0} \circ S_{\omega}^{t}$ is measurable in $\omega$. (The last conclusion uses the theorem that the pointwise limit of measurable functions with values in a metric space is measurable. (cf. for example [22], p. 117)).

As mentioned in Remark 1.27.3 there is no distinction of the point 0 by the product topology. So for all $q \in \mathbb{Z}^{d}$ we can define $\pi_{q} \circ S_{\omega}^{t}$ for all $\omega \in \Omega \backslash \mathcal{N}^{q}$ where $P\left(\mathcal{N}^{q}\right)=0$. In the same way we can define for each $\Lambda \in \mathcal{F} \backslash\{\emptyset\}$ and $\omega \in \Omega \backslash \mathcal{N}^{\Lambda}$ (with $P\left(\mathcal{N}^{\Lambda}\right)=0$ ) maps $\pi_{\Lambda} \circ S_{\omega}^{t} \in \mathcal{C}^{0}\left(M,\left(S^{1}\right)^{\Lambda}\right)$ that depend measurably on $\omega$, and such that $S_{\omega}^{t}(\mathbf{x})$ depends measurably on $(\omega, \mathbf{x})$.

The set

$$
\mathcal{N} \stackrel{\text { def }}{=} \bigcup_{\Lambda \in \mathcal{F} \backslash\{\emptyset\}} \mathcal{N}^{\Lambda}
$$

has $P$-measure zero. So by Lemma 1.28 the map $S_{\omega}^{t}$ is well-defined for $\omega \in \Omega \backslash \mathcal{N}$ and the statements in 3 . hold.

Remark 2.14. -

1. It follows from the proof of Theorem 2.13 that one can define a random dynamical system (cf. [1]), given by the map $[0, \infty) \times \Omega \times M \ni(t, \omega, x) \mapsto S_{\omega}^{t}(x)$ and the shifts $\theta(t)$ on $\Omega$ such that $X_{t_{2}}^{q}\left(\theta\left(t_{1}\right) \omega\right) \stackrel{\text { def }}{=} X_{t_{1}+t_{2}}^{q}(\omega)-X_{t_{1}}^{q}(\omega)$ where $X_{t}^{q}(\omega)$ denotes the number of jumps of $\omega$ at site $q$ in the time interval $(0, t]$.

One can further define for $P$-a.a. $\omega \in \Omega$ the linear operators ' $\circ S_{\omega}^{t}$, , acting on continuous functions and the corresponding transfer operators ' $\mathcal{L}_{\omega}^{t}$ '. So one has operator-valued random variables. However, in the following we consider only the averaged (w.r.t. $\omega$ ) operators.

2. We have defined maps $S_{\omega}^{t} \in \mathcal{C}^{0}(M, M)$ for finite range updating in (74) and a special class of infinite range updatings in (109), using (107). Note that the second class does not include the first. It might be interesting to find more general classes 
of (single site) updating functions for which the limit in (107) exists, or examples where it does not exist.

\subsection{Markov kernels}

In Section 1 we defined the Poisson process $\left(\Omega, \mathcal{A}, P,\left(X_{t}\right)_{t \in[0, T]}\right)$ with parameter $\lambda$ and values in $\mathbb{N}^{\Lambda}$, the measure space $\left(M, \mathcal{B}_{M}, \mu\right)$ and the measurable space $\left(\mathcal{C}^{0}(M, M), \mathcal{B}_{\mathcal{C}^{0}(M, M)}\right)$.

We have nets $\left(S_{\Lambda}^{T}\right)_{\Lambda \in \mathcal{F} \backslash\{\emptyset\}}$ of maps $S_{\Lambda}^{T}: \Omega \backslash \mathcal{N} \rightarrow \mathcal{C}^{0}(M, M)$ with limit $S^{T} \in$ $\mathcal{C}^{0}(M, M)$, and the following statements hold:

1. $S_{\Lambda}^{T}$ and $S^{T}$ are $\left(\mathcal{A}, \mathcal{B}_{\mathcal{C}^{0}(M, M)}\right)$-measurable.

2. $S^{T}$ is the pointwise limit of the net $\left(S_{\Lambda}^{T}\right)_{\Lambda \in \mathcal{F} \backslash\{\emptyset\}}$.

3. For fixed $\mathbf{x} \in M$ the map $S_{\Lambda, .}^{T}(\mathbf{x}): \Omega \rightarrow M$ is $\left(\mathcal{A}, \mathcal{B}_{M}\right)$-measurable.

More precisely, for finite range interaction (cf. Section 2.1) $S^{T}$ was defined in (74) and $S_{\Lambda}^{T}$ in (70). (Now we drop the fixed boundary condition $\xi$ and the " $\sim$, in the notation for convenience.) For infinite range interaction (cf. Section 2.2) we define $S_{\Lambda}^{T}$ in the same way as for finite range interaction and the existence of the limit $S^{T}$ is established in (109). Note that these maps are a priori not defined on a set of $P$-measure zero. For these exceptional $\omega \in \Omega$ we define $S_{\omega}^{T}$ and $S_{\Lambda, \omega}^{T}$ to be equal to the identity on $M$.

Statement 3. follows from measurability w.r.t. $(\omega, \mathbf{x})$ of $S_{\Lambda, \omega}^{T}(\mathbf{x})$ (Proposition 2.4 and Remark 1.29.2 for finite range interaction and statement 3. of Theorem 2.13 and Remark 1.29.2 for infinite range interaction), the fact that one-point-sets in $M$ are measurable, and Fubini's Theorem.

Like in Example 1.18 we set

$$
\begin{aligned}
K_{S}^{T}: M \times \mathcal{B}_{M} & \rightarrow[0,1], \\
K_{S}^{T}(\mathbf{x}, A) & \stackrel{\text { def }}{=} P\left(\left\{\omega: S_{\omega}^{T}(\mathbf{x}) \in A\right\}\right) .
\end{aligned}
$$

The corresponding operator, applied to an $f \in \mathcal{C}^{0}(M)$, is

$$
\begin{aligned}
\left(K_{S}^{T} f\right)(\mathbf{x}) & =\int_{M} K_{S}^{T}(\mathbf{x}, \mathrm{d} \mathbf{y}) f(\mathbf{y}) \\
& =\int_{\Omega} \mathrm{d} P(\omega) f \circ S_{\omega}^{T}(\mathbf{x}) .
\end{aligned}
$$

(125) is the definition (cf. (32)), and (126) is a consequence of (124).

We define analogously the Markov kernels $K_{S, \Lambda}^{T}$ and corresponding operators for the Poisson process with values in $\mathbb{N}^{\Lambda}$.

PROPOSITION 2.15. $-K_{S}^{T}$ and $K_{S, \Lambda}^{T}$ are bounded linear operators on $\mathcal{C}^{0}(M)$.

Proof. - We give the proof for $K_{S}^{T}$. The one for $K_{S, \Lambda}^{T}$ is analogous. Let $\omega \in \Omega$, $f \in \mathcal{C}^{0}(M)$ and $\left(\mathbf{x}^{(n)}\right)_{n \in \mathbb{N}}$ a sequence in $M$ with limit $\mathbf{x}$. Then

$$
\begin{aligned}
\lim _{n \rightarrow \infty} S_{\omega}^{T}\left(\mathbf{x}^{(n)}\right) & =S_{\omega}^{T}(\mathbf{x}) \\
\text { and so } \quad \lim _{n \rightarrow \infty} f \circ S_{\omega}^{T}\left(\mathbf{x}^{(n)}\right) & =f \circ S_{\omega}^{T}(\mathbf{x}) .
\end{aligned}
$$


Further,

$$
\left\|f \circ S_{\omega}^{T}\right\|_{\infty} \leqslant\|f\|_{\infty} .
$$

Using the Dominated Convergence Theorem, we conclude

$$
\begin{aligned}
\lim _{n \rightarrow \infty}\left(K_{S}^{T} f\right)\left(\mathbf{x}^{(n)}\right) & =\lim _{n \rightarrow \infty} \int_{\Omega} \mathrm{d} P(\omega) f \circ S_{\omega}^{T}\left(\mathbf{x}^{(n)}\right) \\
& =\int_{\Omega} \mathrm{d} P(\omega) f \circ S_{\omega}^{T}(\mathbf{x}) \\
& =\left(K_{S}^{T} f\right)(\mathbf{x}) .
\end{aligned}
$$

So $K_{S}^{T} f$ is continuous. Continuity of the operator follows from (126) and (129).

Proposition 2.16. - The net $\left(K_{S, \Lambda}^{T}\right)_{\Lambda \in \mathcal{F} \backslash\{\emptyset\}}$ converges weakly to $K_{S}^{T}\left(\right.$ as $\left.\Lambda \rightarrow \mathbb{Z}^{d}\right)$, i.e. for all $f \in \mathcal{C}^{0}(M)$ :

$$
\lim _{\Lambda \rightarrow \mathbb{Z}^{d}} K_{S, \Lambda}^{T} f=K_{S}^{T} f
$$

Proof. - We have

$$
\left\|K_{S}^{T} f-K_{S, \Lambda}^{T} f\right\|_{\infty} \leqslant \int_{\Omega} \mathrm{d} P(\omega)\left\|f \circ S_{\omega}^{T}-f \circ S_{\Lambda, \omega}^{T}\right\|_{\infty} .
$$

Because of condition 2 on p. 26 and (129) the rhs converges to 0 (as $\Lambda \rightarrow \mathbb{Z}^{d}$ ).

Remark 2.17. - It follows from Remark 2.14.1 and the homogeneity of Poisson processes w.r.t. time that $\left(K_{S}^{t}\right)_{t \geqslant 0}$ is a semigroup.

\section{Transfer operators}

In this section we define transfer operators for the Markov kernels for a special class of updating functions that we have already studied in [13]. First we recall some definitions and notations from [13].

For $\delta>0$ we denote by $A_{\delta}$ the annulus

$$
A_{\delta} \stackrel{\text { def }}{=}\{z \in \mathbb{C}|-\delta \leqslant \ln | z \mid \leqslant \delta\}
$$

and by $\Gamma$ its positively oriented boundary.

For $\emptyset \neq \Lambda \subset \mathbb{Z}^{d}$ the normalized Lebesgue measure on $\left(S^{1}\right)^{\Lambda}$ is denoted by $\mu^{\Lambda}$. For finite $\Lambda$ it is given by

$$
\mathrm{d} \mu^{\Lambda}(\mathbf{z})=\frac{\mathrm{d} \mathbf{z}}{(2 \pi \mathrm{i})^{|\Lambda|}} \frac{1}{\mathbf{z}} \stackrel{\text { def }}{=} \prod_{p \in \Lambda} \frac{\mathrm{d} z_{p}}{2 \pi \mathrm{i}} \frac{1}{z_{p}} .
$$

We also use $\mathrm{d} \mu^{\Lambda}(\mathbf{z})$ as a shorthand notation for the right-hand side of (134) for $\mathbf{z} \in A_{\delta}^{\Lambda}$.

In Assumption I (see below) we will fix a $\delta>0$. For $\Lambda \in \mathcal{F}$ we denote by $\mathcal{H}_{\Lambda}$ the space of continuous functions on the polyannulus $A_{\delta}^{\Lambda}$ that are holomorphic on its interior and 
write $\|\cdot\|_{\Lambda}$ for the uniform norm on $\mathcal{H}_{\Lambda}$. As a function on $A_{\delta}^{\Lambda}$ is also a function on $A_{\delta}^{\mathbb{Z}^{d}}$ we can drop the index $\Lambda$ and mean the uniform norm on the infinite-dimensional polyannulus. $\mathcal{H}$ is the vectorspace of all consistent families $\phi=\left(\phi_{\Lambda}\right)_{\Lambda \in \mathcal{F}}$ of functions $\phi_{\Lambda} \in \mathcal{H}_{\Lambda}$. Consistency means

$$
\begin{aligned}
\left(\pi_{\Lambda_{1}} \phi_{\Lambda_{2}}\right)\left(\mathbf{z}_{\Lambda_{1}}\right) & \stackrel{\text { def }}{=} \int_{\left(S^{1}\right)^{\Lambda_{2} \backslash \Lambda_{1}}} \mathrm{~d} \mu^{\Lambda_{2} \backslash \Lambda_{1}}\left(\mathbf{z}_{\Lambda_{2} \backslash \Lambda_{1}}\right) \phi\left(\mathbf{z}_{\Lambda_{1}} \vee \mathbf{z}_{\Lambda_{2} \backslash \Lambda_{1}}\right) \\
& =\phi_{\Lambda_{1}}
\end{aligned}
$$

for all $\Lambda_{1} \subseteq \Lambda_{2} \in \mathcal{F}$ and $\mathbf{z}_{\Lambda_{1}} \in A_{\delta}^{\Lambda_{1}}$. (Note that we use the same symbol ' $\pi_{\Lambda}$ ' for projections of functions and projections of coordinates, for example from $M$ to $\left(S^{1}\right)^{\Lambda}$.)

For $0<\vartheta<1$ and $\phi \in \mathcal{H}$ we define

$$
\begin{gathered}
\|\phi\|_{\vartheta}=\sup _{\Lambda \in \mathcal{F}} \vartheta^{|\Lambda|}\left\|\phi_{\Lambda}\right\|_{\Lambda}, \\
\|\phi\|_{\text {var }} \stackrel{\text { def }}{=} \lim _{\Lambda \rightarrow \mathbb{Z}^{d}} \int_{\left(S^{1}\right)^{\Lambda}} \mathrm{d} \mu^{\Lambda}\left|\phi_{\Lambda}\right| .
\end{gathered}
$$

We set

$$
\begin{aligned}
& \mathcal{H}_{\vartheta} \stackrel{\text { def }}{=}\left\{\phi \in \mathcal{H}:\|\phi\|_{\vartheta}<\infty\right\}, \\
& \mathcal{H}^{b v} \stackrel{\text { def }}{=}\left\{\phi \in \mathcal{H}:\|\phi\|_{v a r}<\infty\right\}, \\
& \mathcal{H}_{\vartheta}^{b v} \stackrel{\text { def }}{=} \mathcal{H}^{b v} \cap \mathcal{H}_{\vartheta} .
\end{aligned}
$$

Then $\left(\mathcal{H}_{\vartheta},\|\cdot\|_{\vartheta}\right)$ is a Banach space. For $\phi \in \mathcal{H}^{b v}$ and $\psi \in \mathcal{C}^{0}(M)$ we define

$$
\begin{gathered}
\psi_{\Lambda}\left(\mathbf{z}_{\Lambda}\right) \stackrel{\text { def }}{=} \int_{\left(S^{1}\right)^{\Lambda^{C}}} \mathrm{~d} \mu^{\Lambda^{C}}\left(\mathbf{z}_{\Lambda^{c}}\right) \psi\left(\mathbf{z}_{\Lambda} \vee \mathbf{z}_{\Lambda^{c}}\right), \\
\int_{M} \mathrm{~d} \mu \psi \phi \stackrel{\text { def }}{=} \lim _{\Lambda \rightarrow \mathbb{Z}^{d}} \int_{\left(S^{1}\right)^{\Lambda}} \mathrm{d} \mu_{\Lambda} \psi_{\Lambda} \phi_{\Lambda} .
\end{gathered}
$$

Finally we recall the definition of a transfer operator: Let $\tilde{\mu}$ be a measure on the (completed) Borel $\sigma$-algebra of a metric space $\widetilde{M}$ and $\widetilde{S}: \widetilde{M} \rightarrow \widetilde{M}$ be a measurable map that is non-singular w.r.t. $\mu$, i.e. for all measurable $A \in \widetilde{M}, \mu(A)=0$ implies $\mu\left(\widetilde{S}^{-1}(A)\right)=0$. The Perron-Frobenius operator (or transfer operator) $\mathcal{L}_{\widetilde{S}}$, acting on $L^{1}(\widetilde{M})$, is defined via the equation

$$
\int_{\widetilde{M}} \mathrm{~d} \tilde{\mu} \psi \circ \widetilde{S} \phi=\int_{\widetilde{M}} \mathrm{~d} \tilde{\mu} \psi \mathcal{L}_{\widetilde{S}} \phi
$$

that must hold for all $\psi \in L^{\infty}(\widetilde{M})$ and $\phi \in L^{1}(\widetilde{M})$.

The Markov kernels for our stochastic systems are analogous to the composition operator ' $\circ S$ ' (with deterministic $S$ ), acting on functions. 
DEFINITION 3.1. - We define transfer operator for a Markov kernel $K$ analogously to (143) by the equation

$$
\int_{\widetilde{M}} \mathrm{~d} \tilde{\mu}(K \psi) \phi=\int_{\widetilde{M}} \mathrm{~d} \tilde{\mu} \psi\left(\mathcal{L}_{K} \phi\right) .
$$

Remark 3.2.-

1. In the cases we consider, the Markov kernel $K_{S}$ is given by

$$
\left(K_{S} \psi\right)(x)=\int_{\Omega} \mathrm{d} P(\omega) \psi \circ S_{\omega}(x),
$$

where $\Omega$ is a probability space, $S_{\omega}$ depends measurably on $\omega$ and the map $\omega \mapsto \mathcal{L}_{S_{\omega}}$ is well-defined and integrable. Then

$$
\begin{aligned}
\int_{\widetilde{M}} \mathrm{~d} \tilde{\mu}(K \psi) \phi & =\int_{\widetilde{M}} \mathrm{~d} \tilde{\mu}(x) \int_{\Omega} \mathrm{d} P(\omega) \psi \circ S_{\omega}(x) \phi(x) \\
& =\int_{\widetilde{M}} \mathrm{~d} \tilde{\mu}(x) \int_{\Omega} \mathrm{d} P(\omega) \psi(x)\left(\mathcal{L}_{S_{\omega}} \phi\right)(x) \\
& =\int_{\widetilde{M}} \mathrm{~d} \tilde{\mu}(x) \psi(x) \int_{\Omega} \mathrm{d} P(\omega)\left(\mathcal{L}_{S_{\omega}} \phi\right)(x) .
\end{aligned}
$$

So $\mathcal{L}_{K_{S}}$ is given by

$$
\left(\mathcal{L}_{K_{S}} \phi\right)(x)=\int_{\Omega} \mathrm{d} P(\omega)\left(\mathcal{L}_{S_{\omega}} \phi\right)(x) .
$$

2. The operator for the infinite dimensional system that we are going to consider act on elements of $\mathcal{H}_{\vartheta}$ that do not in general correspond to elements of $L^{1}(M)$. Recall (see [13]) that $\mathcal{H}_{\vartheta}^{b v}$ can be identified with a subset of $r c a(M)$ (or, in other words, a subset of the Borel measures). So for example in Theorem 3.25 we will show that the equation analogous to (144) holds for $\psi \in \mathcal{C}^{0}(M)$ (rather than $L^{\infty}(M)$ ) and $\phi \in \mathcal{H}_{\vartheta}^{b v}$.

Now we consider a special class of interactions (cf. [13]), namely a family $\left(S_{\Lambda}\right)_{\Lambda \in \mathcal{F}}$ of maps on $M$ that can be written as

$$
\begin{aligned}
S_{\Lambda}: M & \rightarrow M, \\
S_{\Lambda}(\mathbf{z}) & =F_{\Lambda} \circ T_{\Lambda}(\mathbf{z}) \vee \mathbf{z}_{\Lambda} c,
\end{aligned}
$$

where

$$
\begin{gathered}
F_{\Lambda}:\left(S^{1}\right)^{\Lambda} \rightarrow\left(S^{1}\right)^{\Lambda}, \\
\mathbf{z}_{\Lambda}=\left(z_{q}\right)_{q \in \Lambda} \mapsto\left(f_{q}\left(z_{q}\right)\right)_{q \in \Lambda},
\end{gathered}
$$

and 


$$
\begin{gathered}
T_{\Lambda}: M \rightarrow\left(S^{1}\right)^{\Lambda}, \\
\left(T_{\Lambda}(\mathbf{z})\right)_{q} \stackrel{\text { def }}{=} z_{q} \exp \left(2 \pi \mathrm{i} \varepsilon \sum_{k=1}^{\infty} g_{q, k}(\mathbf{z})\right) \quad \text { for } q \in \Lambda
\end{gathered}
$$

and $f_{q}$ and $g_{q, k}$ satisfy the following assumptions:

Assumption $I .-F(\mathbf{z})=\left(f_{q}\left(z_{q}\right)\right)_{q \in \mathbb{Z}^{d}}$ where $f_{q}: S^{1} \rightarrow S^{1}$ are real analytic and expanding (i.e. $f_{q}^{\prime} \geqslant \lambda_{0}>1$ ) maps that extend for some $\delta_{1}$ holomorphically to the interior of an annulus $A_{\delta_{1}}$. In Proposition 3.1 and 3.2 of [13] we have shown that the holomorphic extension to a sufficiently thin annulus $A_{\delta}$ is expanding in the sense that the preimage of $A_{\delta}$ w.r.t. $f_{q}$ lies in the interior of $A_{\delta}$. We fix such a $\delta_{1}$. Then for every $q \in \mathbb{Z}^{d}$ the Perron-Frobenius operator $\mathcal{L}_{f_{q}}$, acting on $\mathcal{H}_{\{q\}}$, has a simple largest eigenvalue 1 with eigenvector $h_{q}$, such that $\pi_{\emptyset}\left(h_{q}\right)=1$ and the restriction of $h_{q}$ to $S^{1}$ is positive and it splits into

$$
\mathcal{L}_{f_{q}}=\mathcal{Q}_{q}+\mathcal{R}_{q}
$$

where $\mathcal{Q}_{q}$ is a projection onto $\operatorname{span}\left(h_{q}\right)$. We assume that there are positive constants $\eta<1, c_{h}$ and $c_{r}$ such that the following two estimates hold for all $q \in \mathbb{Z}^{d}$ :

$$
\begin{aligned}
& \left\|\mathcal{Q}_{q}\right\|_{\{q\}} \leqslant c_{h}, \\
& \left\|\mathcal{R}_{q}^{n}\right\|_{\{q\}} \leqslant c_{r} \eta^{n},
\end{aligned}
$$

where $\|\cdot\|_{\{q\}}$ denotes both the uniform norm on $\mathcal{H}_{\{q\}}$ (for this we might have to take $\delta_{1}$ even smaller) and the induced operator-norm. We note that this holds in particular if $f_{q}$ does not depend on $q$.

We further have

$$
\mathcal{Q}_{q} \mathcal{R}_{q}=\mathcal{R}_{q} \mathcal{Q}_{q}=0 .
$$

Assumption II. - For all $q \in \mathbb{Z}^{d}$ and $k \geqslant 1$ each map $g_{q, k}$ extends to a holomorphic map $g_{q, k}: A_{\delta_{1}}^{B_{k}(q)} \rightarrow \mathbb{C}$ (recall definition (23) of $B_{k}(q)$ ) and its sup-norm (of modulus) is exponentially bounded by

$$
\left\|g_{q, k}\right\|_{A_{\delta_{1}}^{B_{k}(q)}} \leqslant c_{3} \exp \left(-c_{g} k^{d}\right)
$$

with $c_{3}>0$ and 'large' $c_{g}>0$. (In several statements in Section 3 and 4 a lower bound for $c_{g}$ will come out of our computations. The idea is always that our estimates work, provided $c_{g}$ is bigger than a certain constant.)

For $\Lambda \in \mathcal{F} \backslash\{\emptyset\}$ we denote by $h_{\Lambda}$ the function

$$
h_{\Lambda}\left(\mathbf{z}_{\Lambda}\right) \stackrel{\text { def }}{=} \prod_{q \in \Lambda} h_{q}\left(z_{q}\right),
$$

where $h_{q}$ is as in Assumption I. We set $h_{\emptyset}=1$ and

$$
h_{\mathbb{Z}^{d}} \stackrel{\text { def }}{=}\left(h_{\Lambda}\right)_{\Lambda \in \mathcal{F}} \in \mathcal{H} .
$$


We further define for a fixed $\xi \in M$ and $\Lambda \in \mathcal{F} \backslash\{\emptyset\}$ and $\Lambda_{1} \subseteq \Lambda$ the updating at the $\Lambda_{1}$-sites with fixed boundary conditions $\xi_{\Lambda^{c}}$ outside $\Lambda$ (or cut-off of $S_{\Lambda_{1}}$ ):

$$
\begin{aligned}
S_{\Lambda_{1}, \Lambda}:\left(S^{1}\right)^{\Lambda} & \rightarrow\left(S^{1}\right)^{\Lambda}, \\
\mathbf{z}_{\Lambda} & \mapsto \pi_{\Lambda} \circ S_{\Lambda_{1}}\left(\mathbf{z}_{\Lambda} \vee \xi_{\Lambda^{c}}\right) .
\end{aligned}
$$

And for $\mathbf{z}_{\Lambda \backslash \Lambda_{1}} \in\left(S^{1}\right)^{\Lambda \backslash \Lambda_{1}}$ we define

$$
\begin{aligned}
\pi_{\Lambda_{1}} \circ S_{\Lambda_{1}, \Lambda}\left(\cdot \vee \mathbf{z}_{\Lambda \backslash \Lambda_{1}}\right):\left(S^{1}\right)^{\Lambda_{1}} & \rightarrow\left(S^{1}\right)^{\Lambda_{1}} \\
\mathbf{z}_{\Lambda_{1}} & \mapsto \pi_{\Lambda_{1}} \circ S_{\Lambda_{1}, \Lambda}\left(\mathbf{z}_{\Lambda_{1}} \vee \mathbf{z}_{\Lambda \backslash \Lambda_{1}}\right) .
\end{aligned}
$$

Remark 3.3.-

1. The map defined in (160) is the cut-off of $S$ w.r.t. $\Lambda_{1}$ and boundary conditions $\mathbf{z}_{\Lambda \backslash \Lambda_{1}} \vee \xi_{\Lambda}$. So we can use the special representation in terms of integral kernels for its transfer operator, restricted to $\mathcal{H}_{\Lambda_{1}}$, for the proposition below.

2. The family $\left(S_{q}\right)_{q \in \mathbb{Z}^{d}}$, defined by (148), satisfies conditions (75) and (76) as one can see from [13]: The partial derivatives are estimated in the proof of Proposition 3.1 there.

Lemma 3.4. - Let $\Lambda \in \mathcal{F} \backslash\{\emptyset\}$ be the disjoint union of $\Lambda_{1}$ and $\Lambda_{2}$. The transfer operator, restricted to $\mathcal{H}_{\Lambda_{1}}$, of the map $S_{\Lambda_{1}, \Lambda}:\left(S^{1}\right)^{\Lambda} \rightarrow\left(S^{1}\right)^{\Lambda}$, defined in (159) has the following representation in terms of integral kernels:

$$
\begin{aligned}
& \left(\mathcal{L}_{S_{\Lambda_{1}, \Lambda}} \phi\right)\left(\mathbf{w}_{\Lambda_{1}} \vee \mathbf{w}_{\Lambda_{2}}\right) \\
& \quad=\int_{\Gamma^{\Lambda_{1}}} \mathrm{~d} \mu^{\Lambda_{1}}\left(\mathbf{z}_{\Lambda_{1}}\right) \phi\left(\mathbf{z}_{\Lambda_{1}} \vee \mathbf{w}_{\Lambda_{2}}\right) \prod_{q \in \Lambda_{1}} \frac{\left(S_{\Lambda_{1}, \Lambda}\left(\mathbf{z}_{\Lambda_{1}} \vee \mathbf{w}_{\Lambda_{2}}\right)\right)_{q}}{\left(S_{\Lambda_{1}, \Lambda}\left(\mathbf{z}_{\Lambda_{1}} \vee \mathbf{w}_{\Lambda_{2}}\right)\right)_{q}-w_{q}}
\end{aligned}
$$

for $\phi \in \mathcal{H}_{\Lambda}$.

Proof. - Let $\psi \in \mathcal{C}^{0}\left(\left(S^{1}\right)^{\Lambda}\right)$. We use the notation $\phi_{\mathbf{w}_{\Lambda_{2}}}$ for the function $\mathbf{w}_{\Lambda_{1}} \mapsto$ $\phi\left(\mathbf{w}_{\Lambda_{1}} \vee \mathbf{w}_{\Lambda_{2}}\right)$.

$$
\begin{aligned}
& \int_{\left(S^{1}\right)^{\Lambda}} \mathrm{d} \mu^{\Lambda}\left(\mathbf{w}_{\Lambda}\right) \psi \circ S_{\Lambda_{1}, \Lambda}\left(\mathbf{w}_{\Lambda}\right) \phi\left(\mathbf{w}_{\Lambda}\right) \\
& \quad=\int_{\left(S^{1}\right)^{\Lambda_{2}}} \mathrm{~d} \mu^{\Lambda_{2}}\left(\mathbf{w}_{\Lambda_{2}}\right) \int_{\left(S^{1}\right)^{\Lambda_{1}}} \mathrm{~d} \mu^{\Lambda_{1}}\left(\mathbf{w}_{\Lambda_{1}}\right) \psi_{\mathbf{w}_{\Lambda_{2}}} \circ \pi_{\Lambda_{1}} \circ S_{\Lambda_{1}, \Lambda}\left(\mathbf{w}_{\Lambda_{1}} \vee \mathbf{w}_{\Lambda_{2}}\right) \phi_{\mathbf{w}_{\Lambda_{2}}}\left(\mathbf{w}_{\Lambda_{1}}\right) \\
& =\int_{\left(S^{1}\right)^{\Lambda_{2}}} \mathrm{~d} \mu^{\Lambda_{2}}\left(\mathbf{w}_{\Lambda_{2}}\right) \int_{\left(S^{1}\right)^{\Lambda_{1}}} \mathrm{~d} \mu^{\Lambda_{1}}\left(\mathbf{w}_{\Lambda_{1}}\right) \psi_{\mathbf{w}_{\Lambda_{2}}}\left(\mathbf{w}_{\Lambda_{1}}\right)\left(\mathcal{L}_{\pi_{\Lambda_{1}} \circ S_{\Lambda_{1}, \Lambda}\left(\cdot \mathbf{w}_{\Lambda_{2}}\right)} \phi_{\mathbf{w}_{\Lambda_{2}}}\right)\left(\mathbf{w}_{\Lambda_{1}}\right) \\
& =\int_{\left(S^{1}\right)^{\Lambda}} \mathrm{d} \mu^{\Lambda}\left(\mathbf{w}_{\Lambda}\right) \psi\left(\mathbf{w}_{\Lambda}\right)\left(\mathcal{L}_{\pi_{\Lambda_{1}} \circ S_{\Lambda_{1}, \Lambda}\left(\cdot \vee \mathbf{w}_{\Lambda_{2}}\right.}\right)
\end{aligned}
$$

Using the representation of the transfer operator for $\pi_{\Lambda_{1}} \circ S_{\Lambda_{1}, \Lambda}\left(\cdot \vee \mathbf{w}_{\Lambda_{2}}\right)$ that we established in Proposition 3.3 of [13], we obtain the rhs of (162). 
Remark 3.5.-

1. We see in particular that $\mathcal{L}_{S_{\Lambda_{1}, \Lambda}}$ 'acts on the $\Lambda_{1}$-coordinates' only. There is no integration w.r.t. the $\Lambda_{2}$-coordinates.

For $q \in \Lambda_{1}$ we can split the factor

$$
\begin{aligned}
& \frac{\left(S_{\Lambda_{1}, \Lambda}\left(\mathbf{z}_{\Lambda_{1}} \vee \mathbf{w}_{\Lambda_{2}}\right)\right)_{q}}{\left(S_{\Lambda_{1}, \Lambda}\left(\mathbf{z}_{\Lambda_{1}} \vee \mathbf{w}_{\Lambda_{2}}\right)\right)_{q}-w_{q}} \\
& \quad=h_{q}\left(w_{q}, z_{q}\right)+r_{q}\left(w_{q}, z_{q}\right)+\sum_{k=1}^{\infty} \beta_{q, k}\left(w_{q}, \mathbf{z}_{\Lambda_{1}} \vee \mathbf{w}_{\Lambda_{2}} \vee \xi_{\Lambda^{c}}\right)
\end{aligned}
$$

as in [13]. The integral kernels $h_{q}$ and $r_{q}$ correspond to the operators $\mathcal{Q}_{q}$ and $\mathcal{R}_{q}$, introduced in (152) and $\beta_{q, k}$ to $\mathcal{B}_{q, k}$, say. In addition to (155) we have

$$
\mathcal{Q}_{q} \circ \mathcal{B}_{q, k}=0
$$

for all $k$.

For a detailed analysis on composites of operators $\mathcal{Q}_{q}, \mathcal{R}_{q}, \mathcal{B}_{q, k}$ that have value 0 we refer to Section 5 in [13].

2. We have established a representation of the transfer operator also for updatings at more than one point at one time. Such simultaneous updatings happen, for example, in certain discrete time processes with positive probability (cf. [12]).

As in the systems considered here simultaneous updatings $P$-almost never happen, we can restrict ourself in the following to the case of updatings at single sites, i.e. $\Lambda_{1}=\{q\}$.

DEFinition 3.6. - We define for fixed $\Lambda \in \mathcal{F} \backslash\{\emptyset\}, \xi \in M$ and a finite sequence $j=\left(q_{1}, \ldots, q_{n}\right) \in J$ of points in $\Lambda$ the map

$$
\begin{aligned}
S_{j, \Lambda}:\left(S^{1}\right)^{\Lambda} & \rightarrow\left(S^{1}\right)^{\Lambda}, \\
S_{j, \Lambda} & \stackrel{\text { def }}{=} S_{q_{n}, \Lambda} \circ \cdots \circ S_{q_{1}, \Lambda} .
\end{aligned}
$$

Here $S_{q, \Lambda}$ is the map for the updating at site q. Recall that in Definition 1.22 we defined the maps $\mathbf{j}$. For all $\omega \in \Omega$ there is a finite sequence $\mathbf{j}(\omega)=\left(q_{1}, \ldots, q_{n}\right)$ and so

$$
\mathcal{L}_{S_{\mathbf{j}(\omega), \Lambda}} \stackrel{\text { def }}{=} \mathcal{L}_{S_{q_{n}, \Lambda}} \circ \cdots \circ \mathcal{L}_{S_{q_{1}, \Lambda}}
$$

is well-defined.

Before establishing particular representations of the transfer operators $\mathcal{L}_{\Lambda}^{T}$ and $\pi_{\Lambda} \circ \mathcal{L}^{T}$ we consider some special examples. For these we need the following definition.

DEFINITION 3.7. - We define:

$$
\begin{aligned}
\mathcal{R}(t) & \stackrel{\text { def }}{=} \mathrm{e}^{-\lambda t} \sum_{k=0}^{\infty} \frac{(\lambda t)^{k}}{k !} \mathcal{R}^{k} \\
& =\exp (-\lambda t(\mathrm{id}-\mathcal{R}))
\end{aligned}
$$

Then we have, using (154), 


$$
\begin{aligned}
\mathcal{R}\left(t_{1}\right) \mathcal{R}\left(t_{2}\right) & =\mathcal{R}\left(t_{1}+t_{2}\right), \\
\|\mathcal{R}(t)\| & \leqslant c_{r} \mathrm{e}^{-(1-\eta) \lambda t} .
\end{aligned}
$$

Example 3.8. - Consider a single site system, say at site $q$ of a lattice, with an updating map $f: S^{1} \mapsto S^{1}$ that satisfies Assumption I. We have for fixed time $T>0$ and jump rate $\lambda>0$ a Markov kernel $K_{f}^{T}$, acting on functions $\psi \in \mathcal{C}^{0}\left(S^{1}\right)$ as in (38). Using that $\mathcal{L}_{f}^{n}$ ist the transfer operator of $f^{n}$ (this is a special case of (166)), we get a transfer operator $\mathcal{L}_{f}^{T}$, acting on $\mathcal{H}_{\{q\}}$ (this space is defined on p. 28):

$$
\begin{aligned}
\mathcal{L}_{f}^{T} & =\sum_{n=0}^{\infty} \mathrm{e}^{-\lambda T} \frac{(\lambda T)^{n}}{n !} \mathcal{L}_{f}^{n} \\
& =\sum_{n=0}^{\infty} \mathrm{e}^{-\lambda T} \frac{(\lambda T)^{n}}{n !}(\mathcal{Q}+\mathcal{R})^{n} \\
& =\left(1-\mathrm{e}^{-\lambda T}\right) \mathcal{Q}+\mathcal{R}(T)
\end{aligned}
$$

with $\mathcal{R}(t)$ as in Definition 3.7. Note that we think of the summand $\mathrm{e}^{-\lambda t} \mathrm{id}$, corresponding to $n=0$, as $\mathrm{e}^{-\lambda t} R^{0}$. By (155) we have for $n \geqslant 1$ that $(\mathcal{Q}+\mathcal{R})^{n}=\mathcal{Q}+\mathcal{R}^{n}$, and so we get (172).

We represent the two summands in (172) diagrammatically in Fig. 2. The operator $\left(1-\mathrm{e}^{-\lambda T}\right) \mathcal{Q}$ is represented by a thin vertical line ( $h$-strip) and $\mathcal{R}(T)$ as a thick vertical line ( $r$-strip). Note that the operator $\mathcal{R}(T)$ is a sum of operators, each corresponding to an exponent $0 \leqslant n<\infty$. So the $r$-strip corresponds to that sum of operators rather than to a particular product $\mathrm{e}^{-\lambda T} \frac{(\lambda T)^{n}}{n !} \mathcal{R}^{n}$. An analogous statement holds for the $h$-strip.

Example 3.9. - Now we consider a small perturbation $f_{\varepsilon}$ of the single site system $f_{0}=f$ of Example 3.8, that depends on fixed boundary conditions. For simplicity we split the transfer operator for the single updating into $\mathcal{L}_{f_{\varepsilon}}=\mathcal{Q}+\mathcal{R}+\mathcal{B}$ where $\mathcal{B}$ is the difference between the operators for the perturbed and the unperturbed system. We note that $\mathcal{B}$ corresponds to the sum $\sum_{k=1}^{\infty} \mathcal{B}_{q, k}$ of operators defined in Remark 3.5.1. It follows from (164) that

$$
\mathcal{Q} \circ \mathcal{B}=0
$$

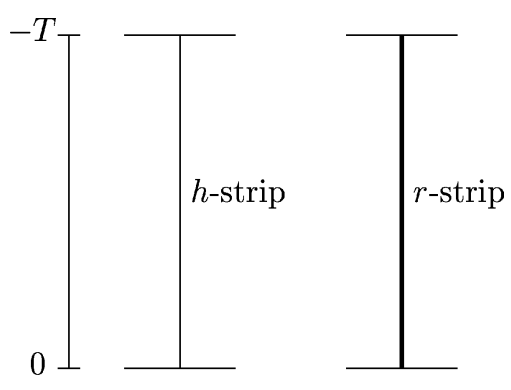

Fig. 2. Single site, unperturbed case: There are only two gum configurations. 
For a given number $N$ of updatings we distribute

$$
\mathcal{L}_{f_{\varepsilon}}^{N}=(\mathcal{Q}+\mathcal{R}+\mathcal{B})^{N},
$$

using (155) and (173). Let $K$ denote the number of factors $\mathcal{B}, n_{0}$ the number of factors, either all $\mathcal{Q}$ or all $\mathcal{R}$, before the first factor $\mathcal{B}$ and $n_{i}$ the number of factors $\mathcal{R}$ after the $i$ th $\mathcal{B}$. So the total number of factors is $N=n_{0}+\cdots+n_{K}+K$.

We get

$$
\begin{aligned}
\mathcal{L}_{f_{\varepsilon}}^{T}= & \sum_{N=0}^{\infty} \mathrm{e}^{-\lambda T} \frac{(\lambda T)^{N}}{N !} \mathcal{L}_{f_{\varepsilon}}^{N} \\
= & \sum_{N=0}^{\infty} \mathrm{e}^{-\lambda T} \sum_{\substack{n_{0}, \ldots, n_{K} \geqslant 0 \\
n_{0}+\cdots+n_{K}+K=N}} \frac{(\lambda T)^{N}}{N !} \mathcal{R}^{n_{K}} \circ \mathcal{B} \circ \cdots \circ \mathcal{B} \circ \mathcal{R}^{n_{1}} \circ \mathcal{B} \circ(\mathcal{Q}+\mathcal{R})^{n_{0}} \\
= & \sum_{K=0}^{\infty} \sum_{n_{n_{0} \geqslant 1}, \ldots, n_{K} \geqslant 0} \mathrm{e}^{-\lambda T} \frac{(\lambda T)^{n_{0}+\cdots+n_{K}+K}}{\left(n_{0}+\cdots+n_{K}+K\right) !} \mathcal{R}^{n_{K}} \circ \mathcal{B} \circ \cdots \circ \mathcal{B} \circ \mathcal{R}^{n_{1}} \circ \mathcal{B} \circ \mathcal{Q} \\
& +\sum_{K=0}^{\infty} \sum_{n_{0}, \ldots, n_{K} \geqslant 0} \mathrm{e}^{-\lambda T} \frac{(\lambda T)^{n_{0}+\cdots+n_{K}+K}}{\left(n_{0}+\cdots+n_{K}+K\right) !} \mathcal{R}^{n_{K}} \circ \mathcal{B} \circ \cdots \circ \mathcal{B} \circ \mathcal{R}^{n_{1}} \circ \mathcal{B} \circ \mathcal{R}^{n_{0}} \\
= & \sum_{K=0}^{\infty} \int_{0}^{T} \lambda \mathrm{d} t_{1} \int_{t_{1}}^{T} \lambda \mathrm{d} t_{2} \cdots \int_{t_{K}}^{T} \lambda \mathrm{d} t_{K}\left(1-\mathrm{e}^{-\lambda t_{1}}\right) \mathcal{R}\left(t_{K}\right) \circ \mathcal{B} \circ \cdots \circ \mathcal{B} \circ \mathcal{R}\left(t_{1}\right) \circ \mathcal{B} \circ \mathcal{Q} \\
& +\sum_{K=0}^{\infty} \int_{0}^{T} \lambda \mathrm{d} t_{1} \int_{t_{1}}^{T} \lambda \mathrm{d} t_{2} \cdots \int_{t_{K}}^{T} \lambda \mathrm{d} t_{K} \mathcal{R}\left(t_{n}\right) \circ \mathcal{B} \circ \cdots \circ \mathcal{R}\left(t_{0}\right)
\end{aligned}
$$

For the step from (177) to (178) we have used Lemma 3.10 (s. below). We interpret (178) in the following way. We write the operator $\mathcal{L}_{f_{\varepsilon}}^{T}$ as a sum of operators $\mathcal{L}_{\mathcal{C}_{g}, T}$. For the time being we think of $\mathcal{C}_{g}$ as a diagram like, for example, in Fig. 3. The vertical axis from top to bottom corresponds to the positively oriented time line. Along this axis we draw $K$ thick horizontal bars, denoted by $B$, that correspond to the operators $\mathcal{B}$. Between consecutive $B$ 's or between the first $B$ and the top or the last $B$ and the bottom or, in the case $K=0$, between the top and the bottom we draw either a thick ( $r$-strip) or a thin ( $h$ strip) line, representing the choice of factors $\mathcal{R}$ or $\mathcal{Q}$ in the product (174), respectively. Note that below a $B$ there must be a thick vertical line. For a fixed $T$ and a $K$-tuple

$$
\mathbf{t}=\left(t_{1}, \ldots, t_{K}\right) \in\left\{\mathbf{t}:-T<t_{1}<\cdots<t_{K}<0\right\}
$$

we think of the top as being fixed at time $-T$, the bottom at 0 and the $i$ th symbol $B$ at $t_{i}$. That also fixes the lengths of the particular $h$ - and $r$-strips. We assign to the triple $\left(\mathcal{C}_{g}, T, t\right)$ an operator $\mathcal{L}_{\mathcal{C}_{g}, T, \mathbf{t}}$. For example with $\mathcal{C}_{g}, T$ and $\mathbf{t}$ as in Fig. 3, we get

$$
\mathcal{L}_{\mathcal{C}_{g}, T, \mathbf{t}}=\left(1-\mathrm{e}^{-\lambda\left(t_{1}+T\right)}\right) \mathcal{R}\left(0-t_{K}\right) \circ \mathcal{B} \circ \cdots \circ \mathcal{B} \circ \mathcal{R}\left(t_{2}-t_{1}\right) \circ \mathcal{B} \circ \mathcal{Q} .
$$




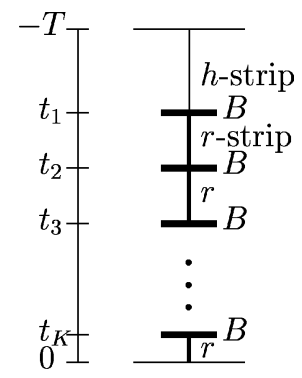

Fig. 3. Single site, perturbed case: example for a gum configuration.

To get $\mathcal{L}_{\mathcal{C}_{g}}^{T}$ we integrate (180) over the simplex given by (179) w.r.t. the scaled Lebesgue measure $\lambda^{K} \mathrm{~d} t_{1} \cdots \mathrm{d} t_{K}$. The simplex has dimension $K$, so for each $B$ in $\mathcal{C}_{g}$ we get one integration. Heuristically, the measure ' $\lambda \mathrm{d} t_{1}$ ' corresponds to the probability that a Poisson process with rate $\lambda$ jumps in a small time interval. The approach of approximating the continuous time system by discrete time systems is made precise in [12]. We also note the special case $K=0$ where the simplex degenerates to a single point of measure 1 .

Above we have used the following lemma.

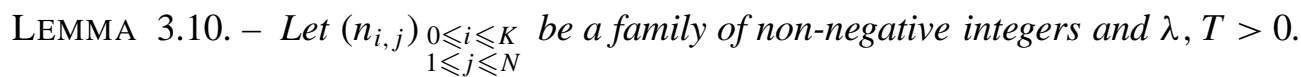
Then, with the notation $t_{0} \stackrel{\text { def }}{=}-T, t_{K+1} \stackrel{\text { def }}{=} 0$ :

$$
\begin{aligned}
\mathrm{e}^{-N \lambda T} \frac{(\lambda T)^{n_{0,1}+\cdots+n_{K, N}+K}}{\left(n_{0,1}+\cdots+n_{K, N}+K\right) !}\left(\begin{array}{c}
n_{0,1}+\cdots+n_{0, N} \\
n_{0,1}, \ldots, n_{0, N}
\end{array}\right) \cdots\left(\begin{array}{c}
n_{K, 1}+\cdots+n_{K, N} \\
n_{K, 1}, \ldots, n_{K, N}
\end{array}\right) \\
=\int_{-T}^{0} \lambda \mathrm{d} t_{1} \int_{t_{1}}^{0} \lambda \mathrm{d} t_{2} \cdots \int_{t_{K-1}}^{0} \lambda \mathrm{d} t_{K} \prod_{\substack{0 \leqslant i \leqslant K \\
1 \leqslant j \leqslant N}}\left(\mathrm{e}^{-\lambda\left(t_{i+1}-t_{i}\right)} \frac{\left(\lambda\left(t_{i+1}-t_{i}\right)\right)^{n_{i, j}}}{n_{i, j} !}\right) .
\end{aligned}
$$

Proof. - We see that the rhs of (181) is equal to

$$
\mathrm{e}^{-N \lambda T} \lambda^{n_{0,1}+\cdots+n_{K, N}+K} \prod_{\substack{0 \leqslant i \leqslant K \\ 1 \leqslant j \leqslant N}} \frac{1}{n_{i, j} !} \int_{-T}^{0} \mathrm{~d} t_{1} \cdots \int_{-t_{K-1}}^{0} \mathrm{~d} t_{K} \prod_{0 \leqslant i \leqslant K}\left(t_{i+1}-t_{i}\right)^{n_{i, 1}+\cdots+n_{i, N}},
$$

and so (181) follows by $K$ times applying the identity

$$
\int_{\tau}^{0} \mathrm{~d} t(-t)^{n}(t-\tau)^{m}=\frac{n ! m !}{(n+m+1) !}(-\tau)^{n+m+1}
$$

which can be easily shown by iterated integration by parts.

Example 3.11. - Now we consider a system with nearest neighbour coupling where only two adjacent sites ( 1 and 2 ) are updated and the states at the other sites are fixed. 


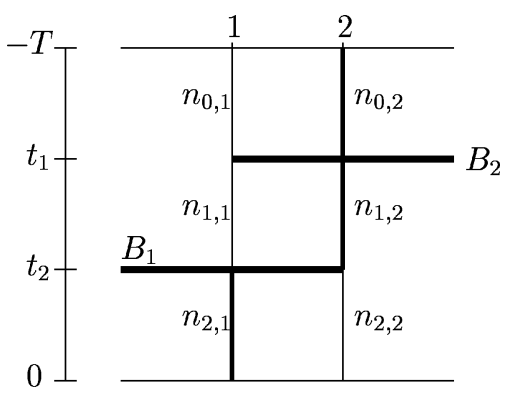

Fig. 4. Two sites, example for a gum configurations.

This time an expansion as in Example 3.9 gives rise to summing operators $\mathcal{L}_{\mathcal{C}_{g}, T}$ where the $\mathcal{C}_{g}$ correspond to diagrams like in Fig. 4.

We have to consider all finite ordered sequences of symbols $B_{1}$ and $B_{2}$.

In the example of Fig. 4 we have the sequence $\left(B_{2}, B_{1}\right)$. For each $B_{i}$ we draw a thick horizontal bar, centered in the column corresponding to the $i$ th site and connecting to all sites (columns) on which the operator $\mathcal{B}_{i}$ depends. We draw the sequence of $B_{i}$ 's 'downwards'. As we consider only nearest neighbour updatings here, the $B_{i}$ have width 2 .

Then we can choose at sites 1 and 2 between $h$ - and $r$-strips. Note that in Fig. 4 at site 2 an $h$-strip follows an $r$-strip. This is possible because they are 'separated' by $B_{1}$ and so the corresponding operator is not necessarily 0. (In Section 5.3 of [13] we list combinations of operators that lead to value 0 .) Now we consider the particular $\mathcal{C}_{g}$ and $T$ shown in Fig. 4. Let $n_{0,1}$ denote the number of jumps at site 1 before the jumps that corresponds to $\mathrm{id}_{1} \otimes \mathcal{B}_{2}$ etc. The definition of $n_{i, j}$ in general is analogous. So the total number of jumps is $N=n_{0,1}+\cdots+n_{2,2}+2$. The first two factors in (184) give the probability that a particular sequence of exactly $N$ jumps occurs. The first binomial coefficient counts the combinations (different sequences) of $n_{0,1}$ jumps at site 1 and $n_{0,2}$ jumps at site 2, the others are explained analogously.

We get the operator:

$$
\begin{aligned}
\mathcal{L}_{\mathcal{C}_{g}, T}= & \sum_{\substack{n_{0,1}, n_{1,1}, n_{2,2} \geqslant 1 \\
n_{0,2}, n_{1,2}, n_{2,1} \geqslant 0}} \mathrm{e}^{-2 \lambda T} \frac{(\lambda T)^{n_{0,1}+\cdots+n_{2,2}+2}}{\left(n_{0,1}+\cdots+n_{2,2}+2\right) !}\left(\begin{array}{c}
n_{0,1}+n_{0,2} \\
n_{0,1}, n_{0,2}
\end{array}\right)\left(\begin{array}{c}
n_{1,1}+n_{1,2} \\
n_{1,1}, n_{1,2}
\end{array}\right) \\
& \left(\begin{array}{c}
n_{2,1}+n_{2,2} \\
n_{2,1}, n_{2,2}
\end{array}\right) \mathcal{R}_{1}^{n_{2,1}} \otimes \mathcal{Q}_{2} \circ \mathcal{B}_{1} \otimes \mathrm{id}_{2} \circ \mathcal{Q}_{1} \otimes \mathcal{R}_{2}^{n_{1,2}} \circ \mathrm{id}_{1} \otimes \mathcal{B}_{2} \circ \mathcal{Q}_{1} \otimes \mathcal{R}_{2}^{n_{0,2}} \\
= & \int_{-T}^{0} \lambda \mathrm{d} t_{1} \int_{t_{1}}^{0} \lambda \mathrm{d} t_{2}\left(\sum_{n_{2,1} \geqslant 0} \mathrm{e}^{-\lambda\left|t_{2}\right|} \frac{\left(\lambda\left|t_{2}\right|\right)^{n_{2,1}}}{n_{2,1} !} \mathcal{R}_{1}^{n_{2,1}}\right) \\
& \otimes\left(\sum_{n_{2,2} \geqslant 1} \mathrm{e}^{-\lambda\left|t_{2}\right|} \frac{\left(\lambda\left|t_{2}\right|\right)^{n_{2,2}}}{n_{2,2} !} \mathcal{Q}_{2}\right) \circ \mathcal{B}_{1} \otimes \mathrm{id}_{2} \\
& \circ\left(\sum_{n_{1,1} \geqslant 1} \mathrm{e}^{-\lambda\left(t_{2}-t_{1}\right)} \frac{\left(\lambda\left(t_{2}-t_{1}\right)\right)^{n_{1,1}}}{n_{1,1} !} \mathcal{Q}_{1}\right)
\end{aligned}
$$




$$
\begin{aligned}
& \otimes\left(\sum_{n_{1,2} \geqslant 0} \mathrm{e}^{-\lambda\left(t_{2}-t_{1}\right)} \frac{\left(\lambda\left(t_{2}-t_{1}\right)^{n_{1,2}}\right.}{n_{1,2} !} \mathcal{R}_{2}^{n_{1,2}}\right) \circ \mathrm{id}_{1} \otimes \mathcal{B}_{2} \\
& \circ\left(\sum_{n_{0,1} \geqslant 1} \mathrm{e}^{-\lambda\left(t_{1}+T\right)} \frac{\left(\lambda\left(t_{1}+T\right)\right)^{n_{0,1}}}{n_{0,1} !} \mathcal{Q}_{1}\right) \\
& \otimes\left(\sum_{n_{0,2} \geqslant 0} \mathrm{e}^{-\lambda\left(t_{1}+T\right)} \frac{\left(\lambda\left(t_{1}+T\right)^{n_{0,2}}\right.}{n_{0,2} !} \mathcal{R}_{2}^{n_{0,2}}\right) \\
= & \int_{-T}^{0} \lambda \mathrm{d} t_{1} \int_{t_{1}}^{0} \lambda \mathrm{d} t_{2}\left(1-\mathrm{e}^{-\lambda\left|t_{2}\right|}\right)\left(1-\mathrm{e}^{-\lambda\left(t_{2}-t_{1}\right)}\right)\left(1-\mathrm{e}^{-\lambda\left(t_{1}+T\right)}\right) \mathcal{R}_{1}\left(\left|t_{2}\right|\right) \otimes \mathcal{Q}_{1} \\
& \circ \mathcal{B}_{1} \otimes \mathrm{id}_{2} \circ \mathcal{Q}_{1} \otimes \mathcal{R}_{2}\left(t_{2}-t_{1}\right) \circ \mathrm{id}_{1} \otimes \mathcal{B}_{2} \circ \mathcal{Q}_{1} \otimes \mathcal{R}_{2}\left(t_{1}+T\right) .
\end{aligned}
$$

Note that the operator ' $\otimes$ ' here has higher precedence than ' $\circ$ ', so, for example, $A_{1} \otimes A_{2} \circ A_{3} \otimes A_{4}$ is understood as $\left(A_{1} \otimes A_{2}\right) \circ\left(A_{3} \otimes A_{4}\right)$.

Remark 3.12. - In these introductory examples we have seen that our transfer operators can be represented as a sum of particular transfer operators $\mathcal{L}_{\mathcal{C}_{g}, T}$ each of whose corresponds to a certain diagram $\mathcal{C}_{g}$ and the time $T$. We will call such a $\mathcal{C}_{g}$ a gum configuration (see Definition 3.16). The $B_{i}$ correspond to particular sets of sites in the lattice (The corresponding integral operator $\mathcal{B}_{i}$ takes these sites into account) and the sequence $\left(\ldots, B_{2}, B_{1}\right)$ reflects a fixed temporal order. Heuristically, we think of the vertical (corresponding to the time coordinate) distances between the $B_{i}$ as as being not yet fixed. The (vertical) $h$ - and $r$-strips of the gum configuration are flexible.

Further, each $\mathcal{L}_{\mathcal{C}_{g}, T}$ can be written as an operator-valued integral where the variable $\mathbf{t}$ of integration is interpreted as time vector and the integrand $\mathcal{L}_{\mathcal{C}_{g}, T, \mathbf{t}}$ corresponds to a specific gum configuration (see Definition 3.19) that can be thought of as the gum configuration $\mathcal{C}_{g}$ whose vertical coordinates are specified by $T$ and $\mathbf{t}$.

Now we establish in a formal way a diagrammatic representation of operators $\pi_{\Lambda_{1}} \circ \mathcal{L}_{S, \Lambda}^{T}$ and $\pi_{\Lambda_{1}} \circ \mathcal{L}_{S}^{T}$.

For that we need some technical definitions and notation. Some of them are taken from [13]. Note that we also use some standard terminology from elementary graph theory here that we assume to be known to the reader.
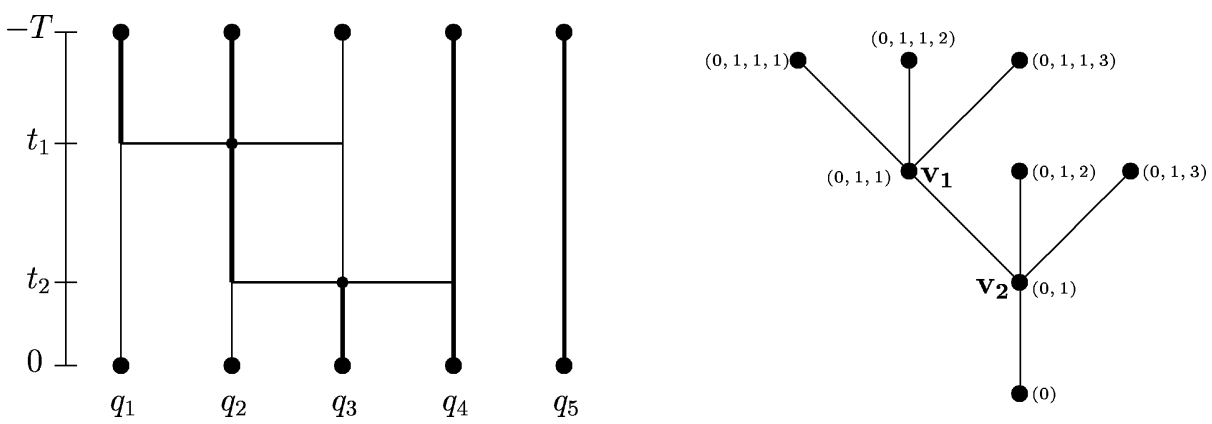

Fig. 5. Specific gum configuration and its labelled tree. 
DEFINITION 3.13 (cf. [13]). - We define the distance between two vertices $v_{1}$ and $v_{2}$ of a connected graph to be the smallest number $l$ such that there is a sequence $\left(w_{0}=v_{1}, w_{1}, \ldots, w_{l}=v_{2}\right)$ of vertices and for all $1 \leqslant i \leqslant l$ the vertices $w_{i}$ and $w_{i-1}$ have a common edge. Two vertices of distance 1 from each other are called neighbours.

A labelled tree is a tree graph whose vertices are labelled in the following way (see also the rhs of Fig. 5 for an example): The root has label (0). If the root has $n$ neighbours then these are labelled by $(0,1), \ldots,(0, n)$, respectively. In general, any vertex $v$ of distance $l$ from the root has a label $\left(0, s_{1}, \ldots, s_{l}\right)$. If such a $v$ has $n$ neighbours each of whose has distance $l+1$ from the root then these neighbours are labelled by $\left(0, s_{1}, \ldots, s_{l}, 1\right), \ldots,\left(0, s_{1}, \ldots, s_{l}, n\right)$, respectively.

We call a vertex of distance $l \geqslant 1$ from the root a leaf if it has no neighbour of distance $l+1$ from the root. All vertices that are not the root or a leaf are called branchings.

For $k \geqslant 1$, we denote by a $k$-branching a branching, say of distance $l$ to the root, that has exactly $v(k)$ neighbours of distance $l+1$ to the root, where $v(k) \stackrel{\text { def }}{=}\left|B_{k}(0)\right|$ and $B_{k}(0)$ is as defined in (23). In this case we kall $k$ the degree of the branching.

In the following we consider only labelled trees, each of whose branchings is of degree $k$ (for some $k$ depending on the particular branching). The number of branchings of degree $k$ is denoted by $n_{\beta, k}$. We collect these numbers in the parameter $n_{\beta} \stackrel{\text { def }}{=}$ $\left(n_{\beta, 1}, n_{\beta, 2}, \ldots\right)$.

A labelled tree that has exactly $K$ vertices of distance 1 from the root and exactly $n_{\beta, k}$ $k$-branchings is called a labelled tree with parameters $K$ and $n_{\beta}$.

(Below and also in [13] a on-to-one-correspondence between the k-branchings of a configuration and the $k$-branchings of the corresponding labelled tree is specified. That explains why we are only interested in labelled trees with those particular branchings.)

The (non-reflexive) linear order $\prec_{v}$ on the set of labels, and so on the set of vertices (of a labelled tree), is generated by the set of all relations of the form $\left(0, s_{1}, \ldots, s_{l}\right) \prec_{v}$ $\left(0, s_{1}, \ldots, s_{l}, i\right)$ or $\left(0, s_{1}, \ldots, s_{l}, i, \ldots\right) \prec_{v}\left(0, s_{1}, \ldots, s_{l}, j, \ldots\right)($ for $i<j)$.

We say that a linear order $\prec_{b}$ on the set of branchings (of a labelled tree) is compatible with the labelling iffor any two branchings the following implication holds:

$$
\operatorname{label}\left(\mathbf{v}_{\mathbf{1}}\right)=\left(0, s_{1}, \ldots, s_{l}\right) \text { and label }\left(\mathbf{v}_{\mathbf{2}}\right)=\left(0, s_{1}, \ldots, s_{l}, i\right) \quad \Rightarrow \quad \mathbf{v}_{\mathbf{2}} \prec_{b} \mathbf{v}_{\mathbf{1}} \text {. }
$$

Finally, we introduce a linear order $\prec$ on $\mathbb{Z}^{d}$ :

$$
\left(k_{1}, \ldots, k_{d}\right) \prec\left(\tilde{k}_{1}, \ldots, \tilde{k}_{d}\right) \quad \text { if } k_{i}<\tilde{k}_{i} \text { for the lowest index } i \text { such that } k_{i} \neq \tilde{k}_{i} .
$$

Definition 3.14. - A gum tree $\tau_{g}$ with parameters $n_{\beta}=\left(n_{\beta, 1}, n_{\beta, 2}, \ldots\right)$ and $\Lambda_{2} \in$ $\mathcal{F} \backslash\{\emptyset\}$ is given by the following data:

1. A labelled tree $\tau$ with parameters $n_{\beta}$ and $\left|\Lambda_{2}\right|$ (as defined in Definition 3.13).

2. A linear order $\prec_{b}$ on the set of branchings of $\tau$ that is compatible with the labelling.

3. A map pin from the set of vertices (except the root) of $\tau$ to $\mathbb{Z}^{d}$ that satisfies the following conditions:

(a) The restriction of pin to the set of vertices, that are labelled by $(0,1), \ldots$, $\left(0,\left|\Lambda_{2}\right|\right)$ (We denote the restriction of pin to this set by $\left.\operatorname{pin}_{0}.\right)$, is an order- 
preserving bijection onto $\Lambda_{2}$, i.e. for any two such vertices $\mathbf{v}$ and $\tilde{\mathbf{v}}$

$$
\operatorname{label}(\mathbf{v}) \prec_{v} \operatorname{label}(\tilde{\mathbf{v}}) \quad \Rightarrow \quad \operatorname{pin}(\mathbf{v}) \prec \operatorname{pin}(\tilde{\mathbf{v}})
$$

(b) If $\mathbf{v}$ with label $(\mathbf{v})=s=\left(s_{1}, \ldots, s_{m}\right)$ is a $k$-branching and $\operatorname{pin}(\mathbf{v})=q \in \mathbb{Z}^{d}$ then the restriction of pin to the set of vertices with labels $(s, 1), \ldots,(s, v(k))$ (We denote the restriction of pin to this set by $\operatorname{pin}_{\mathbf{v}}$.) is an order-preserving bijection onto $B_{k}(q) \subset \mathbb{Z}^{d}$.

(c) If label $\left(\mathbf{v}_{\mathbf{2}}\right)=(0, i)$ then there is no $k$-branching $\mathbf{v}_{\mathbf{3}}$ such that both $\mathbf{v}_{\mathbf{2}} \prec_{b} \mathbf{v}_{\mathbf{3}}$ and $\operatorname{pin}\left(\mathbf{v}_{\mathbf{2}}\right) \in B_{k}\left(\operatorname{pin}\left(\mathbf{v}_{\mathbf{3}}\right)\right)$.

Similarly, if label $\left(\mathbf{v}_{\mathbf{2}}\right)=\left(0, s_{1}, \ldots, s_{l}, i\right)$ is a branching and label $\left(\mathbf{v}_{\mathbf{1}}\right)=$ $\left(0, s_{1}, \ldots, s_{l}\right)$ then there is no $k$-branching $\mathbf{v}_{\mathbf{3}}$ such that both $\mathbf{v}_{\mathbf{2}} \prec_{b} \mathbf{v}_{\mathbf{3}} \prec_{b} \mathbf{v}_{\mathbf{1}}$ and $\operatorname{pin}\left(\mathbf{v}_{\mathbf{3}}\right) \in B_{k}\left(\operatorname{pin}\left(\mathbf{v}_{\mathbf{3}}\right)\right)$.

\section{Remark 3.15. -}

1. Note that for each choice of $\Lambda_{2} \subset \Lambda_{1}$ and a labelled tree with parameters $n_{\beta}$ and $\left|\Lambda_{2}\right|$ the map pin is automatically fixed (by the first two conditions on pin in Definition 3.14). Then it depends on the third condition (on pin) if the given set $\Lambda_{2}$, gum tree $\tau$ and order $\prec_{\mathrm{b}}$ can be assigned to a (unique) gum configuration.

2. Condition 3(c) on the map pin will be justified in the proof of Proposition 3.23 where we assign to the product of operators a (unique by condition 3(c)) gum configuration and hence a gum tree. Also note that in Definition 3.20 we will define operators for a given gum configuration so we will use assignments between operators and diagrammatic data in both directions.

Definition 3.16. - $A$ gum configuration $\mathcal{C}_{g}$ on $\Lambda$ ending in $\Lambda_{1}$ is given by the following data:

1. A gum tree $\tau_{g}$ with parameters $n_{\beta}$ and $\Lambda_{2}$ such that $\Lambda_{2} \subseteq \Lambda_{1}$. The corresponding tree has branchings $\mathbf{v}_{\mathbf{1}} \prec_{b} \cdots \prec_{b} \mathbf{v}_{\mathbf{n}}$, say, with branching-degrees $b_{1}, \ldots, b_{n}$, respectively.

We denote the gum tree of a gum configuration $\mathcal{C}_{g}$ by $\tau_{g}\left(\mathcal{C}_{g}\right)$, the corresponding tree by $\tau\left(\mathcal{C}_{g}\right)$ and its branching parameter by $n_{\beta}\left(\mathcal{C}_{g}\right)$. So the number of branchings is $\left|n_{\beta}\left(\mathcal{C}_{g}\right)\right| \stackrel{\text { def }}{=} \sum_{k=0}^{\infty} n_{\beta, k}\left(\mathcal{C}_{g}\right)$.

2. For each $1 \leqslant i \leqslant n$ there are maps

$$
\begin{aligned}
& u_{i}: B_{b_{i}}\left(\operatorname{pin}\left(\mathbf{v}_{\mathbf{i}}\right)\right) \cap \Lambda \rightarrow\{0,1\}, \\
& d_{i}: B_{b_{i}}\left(\operatorname{pin}\left(\mathbf{v}_{\mathbf{i}}\right)\right) \cap \Lambda \rightarrow\{0,1\}
\end{aligned}
$$

such that

(a) If $q \in B_{b_{i}}\left(\operatorname{pin}\left(\mathbf{v}_{\mathbf{i}}\right)\right) \cap \Lambda$ and $j$ is the smallest number greater than $i$ such that $q \in B_{b_{j}}\left(\operatorname{pin}\left(\mathbf{v}_{\mathbf{j}}\right)\right)$ (if such a $j$ exists at all) then $d_{i}(q)=u_{j}(q)$.

(b) For every $1 \leqslant i \leqslant n$

$$
d_{i}\left(\operatorname{pin}\left(\mathbf{v}_{\mathbf{i}}\right)\right)=1 .
$$

(c) If $q \in B_{b_{i}}\left(\operatorname{pin}\left(\mathbf{v}_{\mathbf{i}}\right)\right) \cap\left(\Lambda \backslash \Lambda_{1}\right)$ and there is no $j>i$ such that $q \in B_{b_{j}}\left(q_{j}\right)$ then $d_{i}\left(q_{i}\right)=0$. 
(We will see later that the maps $u_{i}$ define from a vertex upwards going $h$-strips (if $u_{i}=0$ ) or $r$-strips (if $u_{i}=1$ ). Similarly, the maps $d_{i}$ determine downwards going strips. For a strip between two vertices it should be well-defined if it is an h-strip or an r-strip. Hence we impose condition (a). Condition (b) says that a strip that goes downwards from a branching must be an $r$-strip.)

3. A map long from $\Lambda \backslash \bigcup_{i=1}^{n} B_{b_{i}}\left(\operatorname{pin}\left(\mathbf{v}_{\mathbf{i}}\right)\right)$ to $\{0,1\}$ such that

$$
\operatorname{long}(q)=0 \quad \text { if } q \notin \Lambda_{1} \text {. }
$$

Definition 3.17. - We define in analogy to Definition 5.2 in [13]

$$
\begin{aligned}
& \widetilde{\Lambda}\left(\mathcal{C}_{g}\right) \stackrel{\text { def }}{=} \bigcup_{i=1}^{n} B_{b_{i}}\left(\operatorname{pin}\left(\mathbf{v}_{\mathbf{i}}\right)\right), \\
& \Lambda_{r}\left(\mathcal{C}_{g}\right) \stackrel{\text { def }}{=}\left\{q \in \Lambda \backslash \widetilde{\Lambda}\left(\mathcal{C}_{g}\right): \operatorname{long}(q)=1\right\}, \\
& \Lambda\left(\mathcal{C}_{g}\right) \stackrel{\text { def }}{=} \widetilde{\Lambda}\left(\mathcal{C}_{g}\right) \cup \Lambda_{r}\left(\mathcal{C}_{g}\right) .
\end{aligned}
$$

We introduce the following notation:

- In the situation of 2(a) the point $q$ is the image (w.r.t. pin) of the vertices $\operatorname{pin}_{\mathbf{v}_{\mathbf{i}}}^{-1}(q)$ and $\operatorname{pin}_{\mathbf{v}_{\mathbf{j}}}^{-1}(q)$. We say that $\mathcal{C}_{g}$ has an $h$-strip ( $r$-strip) from $\operatorname{pin}_{\mathbf{v}_{\mathbf{i}}}^{-1}(q)$ to $\operatorname{pin}_{\mathbf{v}_{\mathbf{j}}}^{-1}(q)$ if $d_{i}(q)=0\left(d_{i}(q)=1\right)$. (We note that we do not distinguish the order of the vertices in this notation: A strip from $\mathbf{v}$ to $\tilde{\mathbf{v}}$ is the same as a strip from $\tilde{\mathbf{v}}$ to $\mathbf{v}$.)

- If $q \in B_{b_{i}}\left(\operatorname{pin}\left(\mathbf{v}_{\mathbf{i}}\right)\right) \cap \Lambda$ and $\mathbf{v}=\operatorname{pin}_{\mathbf{v}_{\mathbf{i}}}^{-1}(q)$ and there is no $j>i$ such that $q \in$ $B_{b_{j}}\left(\operatorname{pin}\left(\mathbf{v}_{\mathbf{j}}\right)\right)$ and if $d_{i}(q)=0\left(d_{i}(q)=1\right)$ we say that $\mathcal{C}_{g}$ has an $h$-strip $(r$-strip) from $\mathbf{v}$ to the bottom.

- If $q \in B_{b_{i}}\left(\operatorname{pin}\left(\mathbf{v}_{\mathbf{i}}\right)\right) \cap \Lambda$ and $\mathbf{v}=\operatorname{pin}_{\mathbf{v}_{\mathbf{i}}}^{-1}(q)$ and there is no number $j<i$ such that $q \in B_{b_{j}}\left(\operatorname{pin}\left(\mathbf{v}_{\mathbf{j}}\right)\right)$ and if $u_{i}(q)=0\left(u_{i}(q)=1\right)$ we say that $\mathcal{C}_{g}$ has an $h$-strip ( $r$-strip) from $\mathbf{v}$ to the top.

- In the situation of 2(b) we call the corresponding $r$-strip an apex-r-strip.

- If $q \in \Lambda \backslash \widetilde{\Lambda}\left(\mathcal{C}_{g}\right)$ and $\operatorname{long}(q)=0$ (long $\left.(q)=1\right)$ then we say that $\mathcal{C}_{g}$ has a long $h$ strip (long $r$-strip) at $q$. So $\Lambda_{r}\left(\mathcal{C}_{g}\right) \subset \Lambda_{1}$ is the set of $q$ where $\mathcal{C}_{g}$ has long $r$-strips.

- If $\mathcal{C}_{g}$ has an $r$-strip to the top or a long $r$-strip we say that $\mathcal{C}_{g}$ reaches the top.

We denote by $\operatorname{Conf} g\left(\Lambda, \Lambda_{1}\right)$ the set of all gum configurations on $\Lambda$ ending in $\Lambda_{1}$.

DEFINITION 3.18. - Let $\mathcal{C}_{g}$ be a gum configuration on $\Lambda$ ending in $\Lambda_{1}$ with branchings $\mathbf{v}_{\mathbf{1}} \prec_{b} \ldots \prec_{b} \mathbf{v}_{\mathbf{n}}$ of branching-orders $b_{1}, \ldots, b_{n}$, respectively, and let $T \in$ $(0, \infty]$. Then we define

$$
\operatorname{Simplex}\left(\mathcal{C}_{g}, T\right) \stackrel{\text { def }}{=}\left\{\left(t_{1}, \ldots, t_{n}\right):-T<t_{1}<\cdots<t_{n}<0\right\}
$$

Simplex $\left(\mathcal{C}_{g}, T\right)$ is an open subset of $\mathbb{R}^{n}$ and so carries the induced Lebesgue measure.

For the special case $n_{\beta}\left(\mathcal{C}_{g}\right)=0$ we define $\operatorname{Simplex}\left(\mathcal{C}_{g}, T\right)$ to be a single point having measure 1.

Definition 3.19. $-\operatorname{For}_{g} \in \operatorname{Conf}_{g}\left(\Lambda, \Lambda_{1}\right), T \in(0, \infty]$ and $\mathbf{t} \in \operatorname{Simplex}\left(\mathcal{C}_{g}, T\right)$ we call the triple $\left(\mathcal{C}_{g}, T, \mathbf{t}\right)$ a specific gum configuration. 
Specific gum configurations can be viewed graphically: The vertices are placed in $\mathbb{Z}^{d} \times[-T, 0]$ and the strips are 'spanned' between vertices, the top $(t=-T)$ and the bottom $(t=0)$ :

- We assign to each vertex $\mathbf{v}$ in $\tau\left(\mathcal{C}_{g}\right)$ a point in $\mathbb{Z}^{d} \times[-T, 0]$ in the following way. If $\mathbf{v}_{\mathbf{i}}$ is a branching of degree $b_{i}, q \in B_{b_{i}}\left(\operatorname{pin}\left(\mathbf{v}_{\mathbf{i}}\right)\right)$ and $\mathbf{v}=\operatorname{pin}_{\mathbf{v}_{\mathbf{i}}}^{-1}(q)$ then $\mathbf{v}$ has time-coordinate $t_{i}$. In particular $\mathbf{v}_{\mathbf{i}}$ has time-coordinate $t_{i}$. As further $\operatorname{pin}(\mathbf{v})=q$ we assign $\mathbf{v}$ to $\left(q, t_{i}\right)$.

Let for the following two vertices $\mathbf{v}$ and $\tilde{\mathbf{v}}$ be assigned to $(q, t)$ and $(q, \tilde{t})$, respectively.

- If $\mathcal{C}_{g}$ has an $h$-strip ( $r$-strip) from $\mathbf{v}$ to $\tilde{\mathbf{v}}$ we say that $\left(\mathcal{C}_{g}, T, \mathbf{t}\right)$ has a maximal $h$-strip (maximal $r$ - ${ }^{\circ}$ strip) from $(q, t)$ to $(q, \tilde{t})$. We define its length to be $|t-\tilde{t}|$.

- If $\mathcal{C}_{g}$ has an h-strip ( $r$-strip) from $\mathbf{v}$ to the bottom (this has time-coordinate 0 .) we say that $\left(\mathcal{C}_{g}, T\right.$,t $)$ has a maximal $h$-strip (maximal $r$-strip) from $(q, t)$ to $(q, 0)$. Its length is $|t|$.

- If $\mathcal{C}_{g}$ has an $h$-strip ( $r$-strip) from $\mathbf{v}$ to the top (this has time-coordinate $-T$.) we say that $\left(\mathcal{C}_{g}, T, \mathbf{t}\right)$ has a maximal $h$-strip (maximal $r$-strip) from $(q, t)$ to $(q,-T)$. Its length is $T-|t|$. (Note that for $T=\infty$ this length is $\infty$.)

- If $\mathcal{C}_{g}$ has a long $h$-strip (long $r$-strip) at $q$ we say that $\left(\mathcal{C}_{g}, T, \mathbf{t}\right)$ has a long $h$-strip (long $r$-strip) at $q$. Its length is $T$. (Long $h$-strips (long $r$-strips) are also considered as maximal strips.)

If $\left(\mathcal{C}_{g}, T, \mathbf{t}\right)$ has a maximal h-strip ( $r$-strip) from $\left(q, \tilde{t}_{1}\right)$ to $\left(q, \tilde{t}_{4}\right)$ and $\tilde{t}_{1} \leqslant \tilde{t}_{2}<\tilde{t}_{3} \leqslant \tilde{t}_{4}$ then we say that $\left(\mathcal{C}_{g}, T, \mathbf{t}\right)$ has an h-strip $\left(r\right.$-strip) from $\left(q, \tilde{t}_{2}\right)$ to $\left(q, \tilde{t}_{3}\right)$ (or from $\left(q, \tilde{t}_{3}\right)$ to $\left.\left(q, \tilde{t}_{2}\right)\right)$.

For a branching $\mathbf{v}_{\mathbf{i}}$ and a $q \in B_{b_{i}}\left(\operatorname{pin}\left(\mathbf{v}_{\mathbf{i}}\right)\right)$ we call the maximal h-strip (if any) from $\left(q, t_{i}\right)$ to $(q, t)$ with $t_{i}<t\left(t_{i}>t\right) a$ downwards going (upwards going) $h$-strip associated to the branching. (Note that in our pictures the positively oriented time-axis goes downwards.) The notation for $r$-strips is analogous.

$\left(\mathcal{C}_{g}, T, \mathbf{t}\right)$ must have a downwards going $r$-strip at the points $\left(\operatorname{pin}\left(\mathbf{v}_{\mathbf{i}}\right), t_{i}\right)$ because of condition 2(b). We call it an apex-r-strip.

An h-strip ( $r$-strip) in $\left(\mathcal{C}_{g}, T, \mathbf{t}\right)$ goes to the bottom (to the top) if the corresponding $h$-strip ( $r$-strip) in $\mathcal{C}_{g}$ goes to the bottom (to the top).

We define

$$
\tilde{c}\left(\mathcal{C}_{g}, T, \mathbf{t}\right) \stackrel{\text { def }}{=} \prod_{H}(1-\exp (\lambda \text { length }(H))),
$$

where the product is over all maximal $h$-strips $H$ that do not end in $\left(\Lambda \backslash \Lambda_{1}\right) \times\{0\}$.

We draw in the specific gum configuration in Fig. 5 thick horizontal lines for branchings and thin or thick vertical lines for $h$-strips or $r$-strips, respectively. There are two branchings of degree 1 , at $\left(q_{2}, t_{1}\right)$ and at $\left(q_{3}, t_{2}\right)$. The specific gum configuration has, for example, a long $r$-strip at site $q_{5}$, an $r$-strip from $\left(q_{1}, t_{1}\right)$ to the top and an $h$-strip from $\left(q_{1}, t_{1}\right)$ to the bottom.

Note that the vertices in the labelled gum tree (except the root) are assigned to points in $\mathbb{Z}^{d}$ (in this example $d=1$ ) by the map pin. For example pin $\left(\mathbf{v}_{\mathbf{1}}\right)=q_{2}$. 
DEFINITION 3.20. - We denote by $\mathcal{R}_{q}(t)$ the operator as defined in Definition 3.7, acting on the qth coordinate. For $\mathcal{C}_{g} \in \operatorname{Conf} g\left(\Lambda, \Lambda_{1}\right)$ with $\left|n_{\beta}(\mathcal{C})\right| \geqslant 1$ branchings at $\mathbf{v}_{\mathbf{1}} \prec_{b} \cdots \prec_{b} \mathbf{v}_{\mathbf{n}}$ of degree $b_{1}, \ldots, b_{n}$, respectively, we set $t_{0} \stackrel{\text { def }}{=}-T, t_{n+1} \stackrel{\text { def }}{=} 0$ and define:

$$
\begin{aligned}
& \mathrm{Op}_{1}\left(i, \mathcal{C}_{g}, T, \mathbf{t}\right) \stackrel{\text { def }}{=} \bigotimes_{q \in \Lambda_{\mathcal{Q}}\left(i, \mathcal{C}_{g}\right)} \mathcal{Q}_{q} \bigotimes_{q \in \Lambda_{\mathcal{R}}\left(i, \mathcal{C}_{g}\right)} \mathcal{R}_{q}\left(t_{i+1}-t_{i}\right), \\
& \mathrm{Op}_{2}(i, k) \stackrel{\text { def }}{=} \mathcal{B}_{\operatorname{pin}\left(\mathbf{v}_{\mathbf{i}}\right), k} \bigotimes_{q \in \Lambda \backslash\left\{\operatorname{pin}\left(\mathbf{v}_{\mathbf{i}}\right)\right\}} \mathrm{id}_{q}, \\
& \mathcal{L}_{\mathcal{C}_{g}, \mathbf{t}}^{T} \stackrel{\text { def }}{=} \tilde{c}\left(\mathcal{C}_{g}, T, \mathbf{t}\right) \mathrm{Op}_{1}\left(n, \mathcal{C}_{g}, T, \mathbf{t}\right) \circ \mathrm{Op}_{2}\left(n, b_{n}\right) \circ \cdots \\
& \circ \mathrm{Op}_{1}\left(1, \mathcal{C}_{g}, T, \mathbf{t}\right) \circ \mathrm{Op}_{2}\left(1, b_{1}\right) \circ \mathrm{Op}_{1}\left(0, \mathcal{C}_{g}, T, \mathbf{t}\right), \\
& \text { and } \quad \mathcal{L}_{\mathcal{C}_{g}}^{T} \stackrel{\text { def }}{=} \int_{\operatorname{Simplex}\left(\mathcal{C}_{g}, T\right)} \lambda^{\left|n_{\beta}\left(\mathcal{C}_{g}\right)\right|} \mathrm{d} \mathbf{t} \mathcal{L}_{\mathcal{C}_{g}, \mathbf{t}}^{T},
\end{aligned}
$$

where $\Lambda_{\mathcal{Q}}\left(i, \mathcal{C}_{g}\right)$ is the set of $q \in \Lambda$ such that $\left(\mathcal{C}_{g}, T, \mathbf{t}\right)$ has an $h$-strip from $\left(q, t_{i}\right)$ to $\left(q, t_{i+1}\right)$ and $\Lambda_{\mathcal{R}}\left(i, \mathcal{C}_{g}\right)$ is the set of $q \in \mathbb{Z}^{d}$ such that $\left(\mathcal{C}_{g}, T, \mathbf{t}\right)$ has an $r$-strip from $\left(q, t_{i}\right)$ to $\left(q, t_{i+1}\right)$.

If $n_{\beta}\left(\mathcal{C}_{g}\right)=0$ we simply set

$$
\begin{aligned}
& \mathcal{L}_{\mathcal{C}_{g}, \mathbf{t}}^{T} \stackrel{\text { def }}{=} \bigotimes_{q: \operatorname{long}(q)=0} Q_{q} \bigotimes_{q: \operatorname{long}(q)=1} \mathcal{R}_{q}, \\
& \mathcal{L}_{\mathcal{C}_{g}}^{T} \stackrel{\text { def }}{=} \mathcal{L}_{\mathcal{C}_{g}, \mathbf{t}}^{T} .
\end{aligned}
$$

Finally we set

$$
\pi_{\Lambda_{1}} \circ \mathcal{L}_{S, \Lambda}^{T} \stackrel{\text { def }}{=} \sum_{\mathcal{C}_{g} \in \operatorname{Conf}_{g}\left(\Lambda, \Lambda_{1}\right)} \pi_{\Lambda_{1}} \circ \mathcal{L}_{\mathcal{C}_{g}}^{T}
$$

Remark 3.21.-

1. If $H$ is a maximal $h$-strip from time $t_{i}$ to time $t_{j}$ with $1 \leqslant i<j \leqslant n+1$ then length $(H)=\left|t_{i}-t_{j}\right|$ and so the factor $1-\exp \left(-\lambda\left|t_{i}-t_{j}\right|\right)$ does not depend on $T$. However, in the case $i=0$, i.e. $t_{i}=-T$, the factor $1-\exp \left(-\lambda\left(T-\left|t_{j}\right|\right)\right)$ depends on $T$. For $T=\infty$ this is equal to 1 .

2. From (196), (197), (198) and (199) we see that the map $\mathbf{t} \mapsto \pi_{\Lambda_{1}} \circ \mathcal{L}_{\mathcal{C}_{g}, \mathbf{t}}^{T}$, defined on $\operatorname{Simplex}\left(\mathcal{C}_{g}, T\right)$, is uniformly continuous (because all factors are uniformly continuous w.r.t. t), hence integrable if $T<\infty$. We will see in the next proposition that the integral also exists in the case $T=\infty$. So (200) is well-defined.

3. We see that if $\left(\mathcal{C}_{g}, \infty, \mathbf{t}\right)$ has an $r$-strip going to the top then $\mathcal{L}_{\mathcal{C}_{g}, \mathbf{t}}^{\infty}=0$.

For the following proposition recall that the parameters $\varepsilon$ and $c_{g}$ were introduced in (151) and (156), respectively. The coupling of the interaction between different sites is 'small' if $\varepsilon$ is 'small' and a 'large' $c_{g}$ means 'strong' exponential decay of the interaction (w.r.t. spatial distance). 
PROPOSITION 3.22. - There are constants $0<\tilde{\vartheta}<\vartheta<1$ and a $c_{4}>0$ such that for sufficiently small $\varepsilon>0$, large $c_{g}$, all $T>0, \Lambda_{1} \subset \Lambda \in \mathcal{F} \backslash\{\emptyset\}$ and $\phi \in \mathcal{H}_{\vartheta}$

$$
\tilde{\vartheta}^{\left|\Lambda_{1}\right|} \sum_{\mathcal{C}_{g} \in \operatorname{Conf}_{g}\left(\Lambda, \Lambda_{1}\right)} \int_{\operatorname{Simplex}\left(\mathcal{C}_{g}, T\right)} \lambda^{\left|n_{\beta}\left(\mathcal{C}_{g}\right)\right|} \mathrm{dt}\left\|\pi_{\Lambda_{1}} \circ \mathcal{L}_{\mathcal{C}_{g}}^{T} \phi_{\Lambda}\right\| \leqslant c_{4}\|\phi\|_{\vartheta} .
$$

For sufficiently large $T$ this also holds for suitably chosen $\tilde{\vartheta}=\vartheta$.

Proof. - First we estimate for each $\mathcal{C}_{g} \in \operatorname{Conf}\left(\Lambda, \Lambda_{1}\right)$ and $\mathbf{t} \in \operatorname{Simplex}\left(\mathcal{C}_{g}, T\right)$ the norm $\left\|\pi_{\Lambda_{1}} \circ \mathcal{L}_{\mathcal{C}_{g}, \mathrm{t}}^{T} \phi_{\Lambda}\right\|_{\Lambda_{1}}$. For that we follow the proof of (57) and (58) in [13]. The operators $\mathcal{Q}_{q}, \mathcal{R}_{q}\left(t_{i+1}-t_{i}\right)$ and $\mathcal{B}_{q, k}$ in the representation (199) of the operator $\Lambda_{1} \circ \mathcal{L}_{\mathcal{C}_{g}, \mathrm{t}}^{T}$ as well as the projection operator $\pi_{\Lambda_{1}}$ can be represented by integral operators (see comment after (163) and cf. [13].) Note that by (167), $\mathcal{R}_{q}\left(t_{i+1}-t_{i}\right)$ is the sum of integral operators.

Using the integral representation of the function $\pi_{\Lambda_{1}} \circ \mathcal{L}_{\mathcal{C}_{g}, \mathrm{t}}^{T} \phi_{\Lambda} \in \mathcal{H}_{\Lambda_{1}}$, we proceed as follows:

1. We perform the integration corresponding to all maximal $r$-strips of the specific gum configuration $\left(\mathcal{C}_{g}, T, \mathbf{t}\right)$. In the estimate (205) an $r$-strip $R$ gives rise to a factor $c_{r} \exp (-(1-\eta) \lambda$ length $(R))$ (see (170).)

2. For each maximal $h$-strip that does not end in $\left(\Lambda \backslash \Lambda_{1}\right) \times\{0\}$ we perform the integration $(1-\exp (\lambda \operatorname{length}(H))) h_{q} \cdot \mu_{q}$ (i.e. integration w.r.t. the $q$-coordinate and multiplication by a scalar factor of $h_{q}$.) That leads to a factor $c_{h}(1-$ $\exp (\lambda$ length $(H)))($ see $(153)$.)

3. For all maximal $h$-strips ending in $\left(\Lambda \backslash \Lambda_{1}\right) \times\{0\}$ we perform the integration corresponding to the projection $\pi_{\Lambda_{1}}$ which leads to a factor 1 in the estimate.

4. For each operator $\mathcal{B}_{q, k}$ we estimate the contribution of its integral kernel from above by $\tilde{c}_{3} \varepsilon \exp \left(-c_{g} k^{d}\right)$. That estimate is derived from (156) in the same way as (55) in [13] is proved. In particular, the constant $\tilde{c}_{3}$ is a product of the constant $c_{3}$ in (156) and constants depending on the geometry of the annulus defined in (133) and with parameter $\delta$ as fixed in Assumption I.

5. The integral operator $\pi_{\Lambda_{1}} \circ \mathcal{L}_{\mathcal{C}_{g}, \text { t }}^{T}$ acts on the function $\phi_{\Lambda} \in \mathcal{H}_{\Lambda}$. However, we only have to estimate the norm of $\phi_{\Lambda\left(\mathcal{C}_{g}\right)}$ as $\phi_{\Lambda}$ is simply integrated w.r.t. the $\left(\Lambda \backslash \Lambda\left(\mathcal{C}_{g}\right)\right)$-coordinates, i.e. at least w.r.t. these, possibly also w.r.t. others. To see that, note that the application of the projection operator $\pi_{\Lambda_{1}}$ or $\mathcal{Q}_{q}$ mean integration w.r.t. the $\left(\Lambda \backslash \Lambda_{1}\right)$ - or the $q$-coordinates, respectively.

By $\tilde{n}_{h}$ we denote the number of maximal $h$-strips that have spatial coordinate in $\Lambda\left(\mathcal{C}_{g}\right) \cup \Lambda_{1}$ (for the other $h$-strips there is simply an integration to do, giving rise to a factor 1 in the estimates) and by $\tilde{n}_{r}$ the number of maximal $r$-strips.

Then we get the estimates

$$
\begin{aligned}
\left\|\pi_{\Lambda_{1}} \circ \mathcal{L}_{\mathcal{C}_{g}, \mathbf{t}}^{T} \phi_{\Lambda}\right\|_{\Lambda_{1}} \leqslant & \left(c_{3} \varepsilon\right)^{\left|n_{\beta}\right|} \exp \left(-c_{g} \sum_{k=1}^{\infty} k^{d} n_{\beta, k}\right) c_{h}^{\tilde{n}_{h}} c_{r}^{\tilde{n}_{r}} \\
& \times \prod_{R} \exp (-(1-\eta) \lambda \operatorname{length}(R)) \tilde{c}\left(\mathcal{C}_{g}, T, \mathbf{t}\right)\left\|\phi_{\Lambda\left(\mathcal{C}_{g}\right)}\right\|_{\Lambda\left(\mathcal{C}_{g}\right)},
\end{aligned}
$$


where the product is over all maximal $r$-strips $R$ of $\left(\mathcal{C}_{g}, T, \mathbf{t}\right)$ and $\tilde{c}\left(\mathcal{C}_{g}, T, \mathbf{t}\right)$ is as defined in (196), and

$$
\begin{aligned}
\left\|\phi_{\Lambda\left(\mathcal{C}_{g}\right)}\right\|_{\Lambda\left(\mathcal{C}_{g}\right)} & \leqslant \vartheta^{-\left|\Lambda_{r}\right|-\sum_{k=1}^{\infty}(3 k)^{d} n_{\beta, k}}\|\phi\|_{\vartheta} \\
& \leqslant \vartheta^{-\left|\Lambda_{r}\right|} \prod_{k=1}^{\infty} \vartheta^{-(3 k)^{d} n_{\beta, k}}\|\phi\|_{\vartheta} .
\end{aligned}
$$

Now we consider a labelled tree $\tau$ with parameters $n_{\beta, k}$ and $K$, a set $\Lambda_{2} \subset \Lambda_{1}$ with $\left|\Lambda_{2}\right|=K$ and the set $A\left(\tau, \Lambda_{2}\right)$ of all $\mathcal{C}_{g} \in \operatorname{Conf}_{g}\left(\Lambda, \Lambda_{1}\right)$ whose labelled tree is $\tau$ and whose gum tree has parameter $\Lambda_{2}$. Note that there can be different linear orders on the branchings of $\tau$. We want to estimate

$$
\tilde{\vartheta}^{\left|\Lambda_{1}\right|} \sum_{\mathcal{C}_{g} \in A\left(\tau, \Lambda_{2}\right)} \int_{\operatorname{Simplex}\left(\mathcal{C}_{g}, T\right)} \lambda^{\left|n_{\beta}\left(\mathcal{C}_{g}\right)\right|} \mathrm{d} \mathbf{t}\left\|\pi_{\Lambda_{1}} \circ \mathcal{L}_{\mathcal{C}_{g}, \mathbf{t}}^{T} \phi_{\Lambda}\right\|
$$

and consider this expression as integral over the union of all sets $\operatorname{Simplex}\left(\mathcal{C}_{g}, T\right)$.

We change the variables of integration: Let the branchings of $\tau$ denote by $\tilde{\mathbf{v}}_{\mathbf{1}}, \ldots, \tilde{\mathbf{v}}_{\left|\mathbf{n}_{\beta}\right|}$. A given $\mathcal{C}_{g} \in A\left(\tau, \Lambda_{2}\right)$ has an ordered set of branchings $\mathbf{v}_{\mathbf{1}} \prec_{\mathrm{b}} \cdots \prec_{\mathrm{b}} \mathbf{v}_{\left|\mathbf{n}_{\beta}\right|}$, so that $\mathbf{v}_{\mathbf{i}}=\tilde{\mathbf{v}}_{\mathbf{j}(\mathbf{i})}$, where $j$ is a permutation on the set of indices.

Further, for given $T>0$ and $\mathbf{t} \in \operatorname{Simplex}\left(\mathcal{C}_{g}, T\right)$ the time-coordinate $t_{i}$ corresponds to the branching $\mathbf{v}_{\mathbf{i}}$ whereas we denote by $\tilde{t}_{1}, \ldots, \tilde{t}_{\left|n_{\beta}\right|}$ the lengths of the apex- $r$-strips that correspond to the branching $\tilde{\mathbf{v}}_{\mathbf{1}}, \ldots, \tilde{\mathbf{v}}_{\left|\mathbf{n}_{\beta}\right|}$, respectively. In particular, the $\tilde{t}_{i}$ are bounded by $T$.

For each $\mathbf{t}=\left(t_{1}, \ldots, t_{\left|n_{\beta}\right|}\right) \in \bigcup_{\mathcal{C}_{g} \in A(\tau)} \operatorname{Simplex}\left(\mathcal{C}_{g}, T\right)$ there is a unique $\tilde{\mathbf{t}}=\left(\tilde{t}_{1}, \ldots\right.$, $\left.\tilde{t}_{\left|n_{\beta}\right|}\right)$. The images of the different $\operatorname{simplices} \operatorname{Simplex}\left(\mathcal{C}_{g}, T\right)$ w.r.t. this map are disjoint subsets of $[0, T]^{n}$. Further, the change of variables from $\mathbf{t}$ to $\tilde{\mathbf{t}}$ is linear and has a determinant of modulus 1 . We see that by doing the transformation in several steps: $\tilde{t}_{j(1)}$ is given by a linear equation

$$
\tilde{t}_{j(1)}=\operatorname{Lin}_{1}\left(t_{2}, \ldots, t_{n}\right)-t_{1}
$$

and $\tilde{t}_{j(2)}$ by

$$
\tilde{t}_{j(2)}=\operatorname{Lin}_{2}\left(\tilde{t}_{j(1)}, t_{3}, \ldots, t_{n}\right)-t_{2}
$$

etc. and the statement about the determinant follows. So we can estimate in (207) the term ' $\sum_{\mathcal{C}_{g} \in A\left(\tau, A_{2}\right)} \int_{\operatorname{Simplex}\left(\mathcal{C}_{g}, T\right)} \mathrm{dt} \ldots$. by ' $\int_{[0, T]^{\left|n_{\beta}(\tau)\right|}} \mathrm{d} \tilde{\mathbf{t}} \ldots$...' and so in the estimate of (204) we replace ' $\sum_{\mathcal{C}_{g}} \int_{\operatorname{Simplex}\left(\mathcal{C}_{g}, T\right)} \mathrm{dt} \ldots$..' by ' $\sum_{\Lambda_{2}, \tau} \int_{[0, T]^{\left|n_{\beta}(\tau)\right|}} \mathrm{d} \tilde{\mathbf{t}} \ldots$..' where the sum is over all $\Lambda_{2} \subseteq \Lambda_{1}$ and labelled trees $\tau$ with parameters $\left|\Lambda_{2}\right|$ and $n_{\beta}$.

Next we want to estimate in the last sum the contribution corresponding to all labelled trees with parameter $K$ (and arbitrary $n_{\beta}$ ): For a fixed $0 \leqslant K \leqslant\left|\Lambda_{1}\right|$ there are exactly $\left(\begin{array}{c}\left|\Lambda_{1}\right| \\ K\end{array}\right)$ subsets $\Lambda_{2} \subset \Lambda_{1}$ with $\left|\Lambda_{2}\right|=K$. By Lemma 8.2.(2) of [13], for fixed $\Lambda_{2}$ and $n_{\beta}$ with $\left|n_{\beta}\right| \geqslant\left|\Lambda_{2}\right|$, the number of labelled trees with parameter $\left|\Lambda_{2}\right|$ and $n_{\beta}$ is bounded from above by $4^{\left|\Lambda_{2}\right|} \prod_{k=1}^{\infty}\left(\exp \left(\tilde{c}_{d} k^{d}\right)\right)^{n_{\beta, k}}$, where $\tilde{c}_{d}$ is a constant depending only on the dimension $d$ of the lattice. 
Now we consider a given a set $\Lambda_{2}$, a labelled tree $\tau$ and a choice of $\tilde{\mathbf{t}}$. There is at most one gum tree $\tau_{g}$, having tree $\tau$, such that a specified gum configuration with parameter $\Lambda_{2}$ and gum tree $\tau_{g}$ exists and the $\tilde{t}_{i}$ are the lengths of apex-r-chains. (Then the order on the branches is determined by the $\tilde{t}_{i}$.)

If such a gum tree exists then the (specific) gum configuration is uniquely defined by the choice of up- and downwards going $h$ - and $r$-strips and long $h$ - and $r$-strips.

For each choice of an $h$-strip or $r$-strip we get a factor $c_{h}(1-\exp (\lambda \operatorname{length}(H)))$ or $c_{r} \exp (-(1-\eta) \lambda$ length $(R))$, respectively, as mentioned at the beginning of this proof. So for each branching we can estimate the contribution of constant factors $c_{h}$ and $c_{r}$ of all possible choices from above by a factor $\exp \left(c_{12} k^{d}\right)$. This factor will be compensated for by the factor $\exp \left(-c_{g} k^{d}\right)$ that, as mentioned at the beginning of the proof, from the estimate for the operator $\mathcal{B}_{q, k}$ corresponding to the branching. For that the constant $c_{g}$ has to be sufficiently large.

There are not more than $\left|\Lambda_{1}\right|-\left|\Lambda_{2}\right|$ sites for which we can choose between long $h$ strips and long $r$-strips. A long $r$-strip gives rise to a factor $c_{r} \exp (-(1-\eta) \lambda T)$, and a long $h$-strip to a factor at most $c_{h}$.

Gum configurations $\mathcal{C}_{g}$ without branchings (i.e. $n_{\beta}\left(\mathcal{C}_{g}\right)=0$ ) can only have long rchains (that must end in $\Lambda_{1}$ ) or long h-chains. This case corresponds to the summand for $K=0$ in (211).

We remark that the sum ' $\sum_{n=0}^{\infty}$ ' in (211) also includes the estimate for this special case $K=0$. Then the gum configurations have no branchings and so $n_{\beta}=0$. The sum ' $\sum_{n=0}^{\infty}$ ' should then be replaced by a factor 1 (to avoid confusion). However, this sum is at least 1 and so the estimate is correct.

We estimate the left-hand side (lhs) of (204):

$$
\begin{aligned}
\tilde{\vartheta}^{\left|\Lambda_{1}\right|} & \sum_{\mathcal{C}_{g} \in \operatorname{Confg}\left(\Lambda, \Lambda_{1}\right)} \int_{\operatorname{Simplex}\left(\mathcal{C}_{g}, T\right)} \lambda^{\left|n_{\beta}\left(\mathcal{C}_{g}\right)\right|} \mathrm{dt}\left\|\pi_{\Lambda_{1}} \circ \mathcal{L}_{\mathcal{C}_{g}, t}^{T} \phi_{\Lambda}\right\| \\
\leqslant & \tilde{\vartheta}^{\left|\Lambda_{1}\right|} \sum_{K=0}^{\left|\Lambda_{1}\right|}\left(\begin{array}{c}
\left|\Lambda_{1}\right| \\
K
\end{array}\right)\left(c_{h}+\vartheta^{-1} c_{r} \exp (-(1-\eta) \lambda T)\right)^{\left|\Lambda_{1}\right|-K_{4} 4^{K}} \\
& \times \sum_{n=K}^{\infty}\left(\varepsilon \lambda \sum_{k=1}^{\infty} \exp \left(-c_{g} k^{d}\right) \exp \left(\left(\tilde{c}_{d}+c_{12}\right) k^{d}\right) c^{k^{d}} \vartheta^{-c k^{d}}\right. \\
& \left.\times \int_{0}^{T} \mathrm{~d} t c_{r} \exp (-(1-\eta) \lambda T)\right)^{n}\|\phi\|_{\vartheta} \\
\leqslant & c_{4}\left(\tilde{\vartheta} c_{h}+\frac{\tilde{\vartheta}}{\vartheta} c_{r} \exp (-(1-\eta) \lambda T)+\tilde{\vartheta} \varepsilon_{1}\right)^{\left|\Lambda_{1}\right|}\|\phi\|_{\vartheta}
\end{aligned}
$$

with $\lim _{\varepsilon \rightarrow 0} \varepsilon_{1}=0$. So there are $0<\tilde{\vartheta}<\vartheta$ such that for sufficiently small $\varepsilon$

$$
\tilde{\vartheta} c_{h}+\frac{\tilde{\vartheta}}{\vartheta} c_{r} \exp (-(1-\eta) T)+\tilde{\vartheta} \varepsilon_{1}<1
$$


and so (204) holds uniformly in $\Lambda_{1}$ and $\Lambda$. For sufficiently large $T$ we can choose $\tilde{\vartheta}=\vartheta$ such that (212) holds. So (204) is proved.

Proposition 3.23. $-\mathcal{L}_{S, \Lambda}^{T} \stackrel{\text { def }}{=} \pi_{\Lambda} \circ \mathcal{L}_{S, \Lambda}^{T}$ is the transfer operator, restricted to $\mathcal{H}_{\Lambda}$, for $K_{S, \Lambda}^{T}$, i.e.

$$
\int_{\left(S^{1}\right)^{\Lambda}} \mathrm{d} \mu\left(K_{S, \Lambda}^{T} \psi_{\Lambda}\right) \phi_{\Lambda}=\int_{\left(S^{1}\right)^{\Lambda}} \mathrm{d} \mu \psi_{\Lambda}\left(\mathcal{L}_{S, \Lambda}^{T} \phi_{\Lambda}\right) .
$$

For all $\psi \in \mathcal{C}^{0}\left(\left(S^{1}\right)^{\Lambda}\right)$ and $\phi_{\Lambda} \in \mathcal{H}_{\Lambda}$ the operator $\pi_{\Lambda_{1}} \circ \mathcal{L}_{S, \Lambda}^{T}$ is the composite of $\pi_{\Lambda_{1}}$ and $\mathcal{L}_{S, \Lambda}^{T}$ for any $\Lambda_{1} \subset \Lambda$.

Proof. - The first claim is a special case of the last statement. Note that $\pi_{\Lambda_{1}} \circ \mathcal{L}_{S, \Lambda}^{T}$ is a priori the operator defined in (203). Now we prove that it is actually the composite of $\pi_{\Lambda_{1}}$ and the transfer operator, restricted to $\mathcal{H}_{\Lambda}$, for $K_{S, \Lambda}^{T}$. The convergence of the following expressions follows from Proposition 3.22.

We consider all ordered finite sequences of jump-sites in $\Lambda$. A particular sequence $\left(q_{1}, \ldots, q_{N}\right)$ occurs in a fixed time interval $(-T, 0)$ with probability $\mathrm{e}^{-|\Lambda| \lambda T} \frac{(\lambda T)^{N}}{N !}$ because this is the probability of having exactly $N$ jumps in total, divided by the number $|\Lambda|^{N}$ of different sequences of length $k$ (which have all the same probability).

The sequence corresponds to a map $S_{q_{N}} \circ \cdots \circ S_{q_{1}}$ (cf. Definition 3.6) and so by (166), to a transfer operator

$$
\pi_{\Lambda_{1}} \circ \mathcal{L}_{S_{q_{N}}} \circ \cdots \circ \mathcal{L}_{S_{q_{1}}} .
$$

So the composite of $\pi_{\Lambda_{1}}$ and the transfer operator for $K_{S, \Lambda}^{T}$ is equal to the following sum over all (possibly empty) sequences:

$$
\sum_{\left(q_{1}, \ldots, q_{N}\right)} \mathrm{e}^{-|\Lambda| \lambda T} \frac{(\lambda T)^{N}}{N !} \pi_{\Lambda_{1}} \circ \mathcal{L}_{S_{q_{N}}} \circ \cdots \circ \mathcal{L}_{S_{q_{1}}}
$$

because this is equal to the rhs of (147). The probability space $\Omega$ is partitioned into countably many sets, each corresponding to a particular sequence of jump sites. So we can write the integral here as a weighted sum.

The factors in (214) can be split

$$
\mathcal{L}_{S_{q}}=\left(\mathcal{Q}_{q}+\mathcal{R}_{q}+\sum_{l=1}^{\infty} \mathcal{B}_{q, l}\right) \otimes \mathrm{id}_{\Lambda \backslash\{q\}} .
$$

Expanding the product in (214), we get a sum of operators. Recall the rules (155) and (164), and also that we have, with $\mu_{q}$ denoting the integration w.r.t. the normalized Lebesgue measure $\mu^{\{q\}}$ (as defined in (134)),

$$
\begin{aligned}
\mu_{q} \circ \mathcal{R}_{q} & =0, \\
\mu_{q} \circ \mathcal{B}_{q, l} & =0,
\end{aligned}
$$

for all $q \in \Lambda$ and $l \geqslant 1$ (as $\mathcal{Q}_{q}=h_{q} \cdot \mu_{q}$ ). So some of the summands in the expansion are zero, namely if $\mathcal{Q}_{q}$ is followed by $\mathcal{R}_{q}$, or $\mathcal{R}_{q}$ or $\mathcal{B}_{q}$ are followed by $\mathcal{Q}_{q}$ or a 
projection $\pi_{\Lambda_{1}}$ with $q \notin \Lambda_{1}$. 'Following' here means that there is in between no $\mathcal{B}_{q, l}$ with $\|\tilde{q}-q\| \leqslant l$. In the following we rule out these combinations. (Compare this to the notion of non-zero configurations in [13].)

Now we represent each summand

$$
\pi_{\Lambda_{1}} \circ A_{N} \circ \cdots \circ A_{1}
$$

in the expansion of (214) which is the composite of operators $\pi_{\Lambda_{1}}, \mathcal{B}_{q} \otimes \mathrm{id}_{\Lambda \backslash\{q\}}$, $\mathcal{R}_{q} \otimes \operatorname{id}_{\Lambda \backslash\{q\}}$ and $\mathcal{Q}_{q} \otimes \operatorname{id}_{\Lambda \backslash\{q\}}$ with variable $q \in \Lambda$, by a gum configuration as follows:

We define the obvious order of the factors in (218) such that $A_{1}$ comes before $A_{2}$ etc. Each $\mathcal{B}_{q, l}$ corresponds to a $l$-branching (which is assigned by the map pin to $q$ ) and the order of the operators $\mathcal{B}_{q, l}$ defines the linear order of the branchings. The other factors $\mathcal{Q}_{q}$ and $\mathcal{R}_{q}$ determine the $h$ - and $r$-strips in the following way:

1. Strips between two vertices: Let $\mathbf{v}_{i} \prec_{\mathrm{b}} \mathbf{v}_{j}$ be two branchings of degree $b_{i}$ and $b_{j}$, respectively, and $q \in B_{b_{i}}\left(\operatorname{pin}\left(\mathbf{v}_{i}\right)\right) \cap B_{b_{j}}\left(\operatorname{pin}\left(\mathbf{v}_{j}\right)\right) \cap \Lambda$ such that there is no other branching $\mathbf{v}_{k}$, of degree $b_{k}$, say, with $\mathbf{v}_{i} \prec_{\mathrm{b}} \mathbf{v}_{k} \prec_{\mathrm{b}} \mathbf{v}_{j}$ and $q \in B_{b_{k}}\left(\operatorname{pin}\left(\mathbf{v}_{k}\right)\right)$. Then $\mathcal{C}_{g}$ has an $h$-strip between $\operatorname{pin}_{\mathbf{v}_{i}}^{-1}(q)$ and $\operatorname{pin}_{\mathbf{v}_{j}}^{-1}(q)$ if there is a factor $\mathcal{Q}_{q} \otimes \operatorname{id}_{\Lambda \backslash\{q\}}$ in (218) between the two factors corresponding to $\mathbf{v}_{i}$ and $\mathbf{v}_{j}$. Otherwise $\mathcal{C}_{g}$ has an $r$-strip there (even if there is no factor $\mathcal{R}_{q} \otimes \mathrm{id}_{\Lambda \backslash\{q\}}$.)

2. Strips from a vertex and the top: Let $\mathbf{v}_{j}$ be a $b_{j}$-branching and $q \in B_{b_{j}}\left(\operatorname{pin}\left(\mathbf{v}_{j}\right)\right)$. Assume there is no $b_{i}$-branching $\mathbf{v}_{i}$ with $\mathbf{v}_{i} \prec_{\mathrm{b}} \mathbf{v}_{j}$ and $q \in B_{b_{i}}\left(\operatorname{pin}\left(\mathbf{v}_{i}\right)\right)$. Then $\mathcal{C}_{g}$ has an $h$-strip from $\operatorname{pin}_{\mathbf{v}_{j}}^{-1}(q)$ to the top if there is a factor $\mathcal{Q}_{q} \otimes \operatorname{id}_{\Lambda \backslash\{q\}}$ in (218) before the factor corresponding to $\mathbf{v}_{j}$. Otherwise $\mathcal{C}_{g}$ has an $r$-strip there.

3. Strips from a vertex and the bottom: Let $\mathbf{v}_{i}$ be a $b_{i}$-branching and $q \in B_{b_{i}}\left(\operatorname{pin}\left(\mathbf{v}_{i}\right)\right)$. Assume there is no $b_{j}$-branching $\mathbf{v}_{j}$ with $\mathbf{v}_{i} \prec_{\mathrm{b}} \mathbf{v}_{j}$ and $q \in B_{b_{j}}\left(\operatorname{pin}\left(\mathbf{v}_{j}\right)\right)$. Then $\mathcal{C}_{g}$ has an $h$-strip from $\operatorname{pin}_{\mathbf{v}_{i}}^{-1}(q)$ to the bottom if there is a factor $\mathcal{Q}_{q} \otimes \operatorname{id}_{\Lambda \backslash\{q\}}$ in (218) after the factor corresponding to $\mathbf{v}_{j}$ or if $q \in \Lambda \backslash \Lambda_{1}$. Otherwise $\mathcal{C}_{g}$ has an $r$-strip there.

4. Long strips: Let $q \in \Lambda$ and assume that there is no $b_{i}$-branching $\mathbf{v}_{i}$ with $q \in$ $B_{b_{i}}\left(\operatorname{pin}\left(\mathbf{v}_{i}\right)\right)$. Then $\mathcal{C}_{g}$ has a long $h$-strip at site $q$ if there is a factor $\mathcal{Q}_{q} \otimes \mathrm{id}_{\Lambda \backslash\{q\}}$ in (218) or if $q \in \Lambda \backslash \Lambda_{1}$. Otherwise $\mathcal{C}_{g}$ has a long $r$-strip there.

The assignment of a summand in the sequence (218) of operators to a gum configuration is not injective, as we have already seen in the simple Example 3.8. Now we consider a fixed gum configuration $\mathcal{C}_{g}$ on $\Lambda$ ending in $\Lambda_{1}$ and all sequences corresponding to it. We assume $\mathcal{C}_{g}$ to have at least one branching. The case of no branching is treated in a similar but easier way. To keep the notation simpler, let $\Lambda=\{1, \ldots,|\Lambda|\}$. Any such sequence has the factors $\mathcal{B}_{q_{i}, l_{i}}(i=1, \ldots, K)$ that correspond $1-1$ and order preserving to the $K$ branchings of $\mathcal{C}_{g}$ as described above. Between two consecutive factors $\mathcal{B}_{q_{i}, l_{i}}$ and $\mathcal{B}_{q_{i+1}, l_{i+1}}$ there can be factors $\mathcal{Q}_{q} \otimes \operatorname{id}_{\Lambda \backslash\{q\}}$ (factors $\mathcal{R}_{q} \otimes \mathrm{id}_{\Lambda \backslash\{q\}}$ ) if for any $\mathbf{t} \in \operatorname{Simplex}\left(\mathcal{C}_{g}, T\right)$ the specific gum configuration $\left(\mathcal{C}_{g}, T, \mathbf{t}\right)$ has an $h$-strip (an $r$-strip) from $\left(q, t_{i}\right)$ to $\left(q, t_{i+1}\right)$. Similarly, the option of having such factors before $\mathcal{B}_{q_{1}, l_{1}}$ or after $\mathcal{B}_{q_{K}, l_{K}}$ depends on the $h$-strips and $r$-strips of $\mathcal{C}_{g}$ in the obvious way. Further, such factors belong to particular maximal $r$-strips or $h$-strips in the obvous way.

Let us denote the number of factors $\mathcal{Q}_{q} \otimes \mathrm{id}_{\Lambda \backslash\{q\}}$ or $\mathcal{R}_{q} \otimes \mathrm{id}_{\Lambda \backslash\{q\}}$ before $\mathcal{B}_{q_{1}, l_{1}}$ by $n_{0, q}$ and the number of such factors after $\mathcal{B}_{q_{i}, l_{i}}$ by $n_{i, q}$. 
Note that for every maximal $h$-strip, at site $q$,say, that does not go to the bottom at a site in $\Lambda \backslash \Lambda_{1}$, there must be at least one factor $Q_{q} \otimes \operatorname{id}_{\Lambda \backslash\{q\}}$ because of rules 1,3 and 4 on p. 47. We denote this condition on the family $\left(n_{i, q}\right)_{\substack{0 \leqslant i \leqslant K \\ q \in \Lambda}}$ of numbers by condition A. In total there are

$$
N=\sum_{q \in \Lambda} \sum_{i=0}^{K} n_{i, q}+K
$$

factors that correspond to a particular sequence of $N$ jump sites and such a sequence occurs with probability $\mathrm{e}^{-|\Lambda| \lambda T} \frac{(\lambda T)^{N}}{N !}$. The $\sum_{q \in \Lambda} n_{0, q}$ factors before $\mathcal{B}_{q_{1}, l_{1}}$ can occur in any of the $\left(\begin{array}{c}n_{0,1}+\cdots+n_{0,|\Lambda|} \\ n_{0,1}, \ldots, n_{0,|\Lambda|}\end{array}\right)$ different orders, that all have the same probability.

So if we sum over all (products of) operators that correspond to $\mathcal{C}_{g}$, weighted with the probability that the corresponding sequence of jumps (in the underlying Poisson process) occurs, we get

$$
\begin{aligned}
& \sum_{\substack{\left(n_{i, q}\right) \\
\text { condition A }}} \mathrm{e}^{-|\Lambda| \lambda T} \frac{(\lambda T)^{N}}{N !}\left(\begin{array}{c}
n_{0,1}+\ldots+n_{0,|\Lambda|} \\
n_{0,1}, \ldots, n_{0,|\Lambda|}
\end{array}\right) \cdots\left(\begin{array}{c}
n_{K, 1}+\cdots+n_{K,|\Lambda|} \\
n_{K, 1}, \ldots, n_{K,|\Lambda|}
\end{array}\right) \\
& \times A_{K, 1}^{n_{K, 1}} \otimes \cdots \otimes A_{K,|\Lambda|}^{K, n_{|\Lambda|}} \circ \mathcal{B}_{q_{K}, l_{K}} \otimes \operatorname{id}_{\Lambda \backslash\left\{q_{K}\right\}} \circ \cdots \circ \mathcal{B}_{q_{1}, l_{1}} \otimes \operatorname{id}_{\Lambda \backslash\left\{q_{1}\right\}} \\
& \circ A_{0,1}^{n_{0,1}} \otimes \cdots \otimes A_{0,|\Lambda|}^{n_{0,|\Lambda|}} \\
& =\sum_{\substack{\left(n_{i, q}\right) \\
\text { condition A }}} \int_{\operatorname{Simplex}\left(\mathcal{C}_{g}, T\right)} \lambda^{K} \mathrm{~d} \mathbf{t}\left(\mathrm{e}^{-\lambda\left(-t_{K}\right)} \frac{\left(\lambda\left(-t_{K}\right)\right)^{n_{K, 1}}}{n_{K, 1} !} A_{K, 1}^{n_{K, 1}}\right) \otimes \cdots \\
& \otimes\left(\mathrm{e}^{-\lambda\left(-t_{K}\right)} \frac{\left(\lambda\left(-t_{K}\right)\right)^{n_{K},|\Lambda|}}{n_{K,|\Lambda|} !} A_{K,|\Lambda|}^{n_{K}, \mid \Lambda}\right) \circ \mathcal{B}_{q_{K}, l_{K}} \otimes \operatorname{id}_{\Lambda \backslash\left\{q_{K}\right\}} \circ \cdots \circ \mathcal{B}_{q_{1}, l_{1}} \otimes \operatorname{id}_{\Lambda \backslash\left\{q_{1}\right\}} \\
& \circ\left(\mathrm{e}^{-\lambda\left(t_{1}+T\right)} \frac{\left(\lambda\left(t_{1}+T\right)\right)^{n_{0,1}}}{n_{0,1} !} A_{0,1}^{n_{0,1}}\right) \otimes \cdots \otimes\left(\mathrm{e}^{-\lambda\left(t_{1}+T\right)} \frac{\left(\lambda\left(t_{1}+T\right)\right)^{n_{0,|\Lambda|}}}{n_{0,|\Lambda|} !} A_{0,|\Lambda|}^{n_{0,|\Lambda|}}\right) \\
& =\int_{\operatorname{Simplex}\left(\mathcal{C}_{g}, T\right)} \lambda^{K} \mathrm{~d} \mathbf{t} \prod_{H}\left(1-\mathrm{e}^{-\lambda \text { length }(H)}\right)\left(\sum_{n_{K, 1} \geqslant 0} \mathrm{e}^{-\lambda\left(-t_{K}\right)} \frac{\left(\lambda\left(-t_{K}\right)\right)^{n_{K, 1}}}{n_{K, 1} !} A_{K, 1}^{n_{K, 1}}\right) \otimes \cdots \\
& \otimes\left(\sum_{n_{K,|\Lambda|} \geqslant 0} \mathrm{e}^{-\lambda\left(-t_{K}\right)} \frac{\left(\lambda\left(-t_{K}\right)\right)^{n_{K,|\Lambda|}}}{n_{K,|\Lambda|} !} A_{K,|\Lambda|}^{n_{K} \mid \Lambda \Lambda}\right) \\
& \circ \mathcal{B}_{q_{K}, l_{K}} \otimes \operatorname{id}_{\Lambda \backslash\left\{q_{K}\right\}} \circ \cdots \circ \mathcal{B}_{q_{1}, l_{1}} \otimes \operatorname{id}_{\Lambda \backslash\left\{q_{1}\right\}} \circ\left(\sum_{n_{0,1} \geqslant 0} \mathrm{e}^{-\lambda\left(t_{1}+T\right)} \frac{\left(\lambda\left(t_{1}+T\right)\right)^{n_{0,1}}}{n_{0,1} !} A_{0,1}^{n_{0,1}}\right) \\
& \otimes \cdots \otimes\left(\sum_{n_{0,|\Lambda|} \geqslant 0} \mathrm{e}^{-\lambda\left(t_{1}+T\right)} \frac{\left(\lambda\left(t_{1}+T\right)\right)^{n_{0,|\Lambda|}}}{n_{0,|\Lambda|} !} A_{0,|\Lambda|}^{n_{0,|\Lambda|}}\right) \\
& =\mathcal{L}_{\mathcal{C}_{g}}^{T} .
\end{aligned}
$$

In (220) each operator $A_{k, q}$ stands for either $\mathcal{Q}_{q}$ or $\mathcal{R}_{q}$.

We have interchanged summation with integration and multiplication and applied the following computations to each set of factors belonging to the same maximal $h$-strip that 
does not end in $\left(\Lambda \backslash \Lambda_{1}\right) \times\{0\}$. For notational simplicity, let the maximal $h$-strip under consideration cover the time intervals $\left(t_{0}, t_{1}\right), \ldots,\left(t_{l-1}, t_{l}\right)$. So its length is $t_{l}-t_{0}$. And let the operators $\mathcal{Q}_{q}$ have exponents $n_{1}, \ldots, n_{l}$ :

$$
\begin{aligned}
& \sum_{\substack{n_{0}, \ldots, n_{l} \geqslant 0 \\
n_{0}+\ldots+n_{l} \geqslant 1}} \prod_{i=1}^{l}\left(\mathrm{e}^{-\lambda\left(t_{i}-t_{i-1}\right)} \frac{\left(\lambda\left(t_{i}-t_{i-1}\right)\right)^{n_{i}}}{n_{i} !} \mathcal{Q}_{q}\right) \\
& =\prod_{i=1}^{l}\left(\sum_{n_{i}=0}^{\infty} \mathrm{e}^{-\lambda\left(t_{i}-t_{i-1}\right)} \frac{\left(\lambda\left(t_{i}-t_{i-1}\right)\right)^{n_{i}}}{n_{i} !} \mathcal{Q}_{q}\right)-\prod_{i=1}^{l} \mathrm{e}^{-\lambda\left(t_{i}-t_{i-1}\right)} \mathcal{Q}_{q} \\
& =\left(1-\mathrm{e}^{-\lambda\left(t_{l}-t_{0}\right)}\right) Q_{q} .
\end{aligned}
$$

This explains the appearance of the factor (196) in (221). Recall that each operator $A_{i, q}$ in (221) stands for either $\mathcal{Q}_{q}$ or $\mathcal{R}_{q}$. So we can replace

$$
\begin{aligned}
& \sum_{n_{i, q} \geqslant 0} \mathrm{e}^{-\lambda\left(t_{i}-t_{i-1}\right)} \frac{\left(\lambda\left(t_{i}-t_{i-1}\right)\right)^{n_{i, q}}}{n_{i, q} !} \mathcal{Q}_{q}^{n_{i, q}}=\mathcal{Q}_{q}, \\
& \sum_{n_{i, q} \geqslant 0} \mathrm{e}^{-\lambda\left(t_{i}-t_{i-1}\right)} \frac{\left(\lambda\left(t_{i}-t_{i-1}\right)\right)^{n_{i, q}}}{n_{i, q} !} \mathcal{R}_{q}^{n_{i, q}}=\mathcal{R}_{q}\left(t_{i}-t_{i-1}\right)
\end{aligned}
$$

and get (221).

So we have seen that (215) is equal to $\pi_{\Lambda_{1}} \circ \mathcal{L}_{S, \Lambda}^{T}$, as defined in (203).

For the representation of the transfer operator for the infinite dimensional system we need the following definition.

Definition 3.24. - Let $\Lambda_{1}, \Lambda_{2} \subseteq \Lambda \in \mathcal{F} \backslash\{\emptyset\}$ and $\mathcal{C}_{g} \in \operatorname{Conf} g\left(\Lambda, \Lambda_{1}\right)$. We say that $\mathcal{C}_{g}$ lies in $\Lambda_{2}$ if $\Lambda\left(\mathcal{C}_{g}\right) \cup \Lambda_{1} \subseteq \Lambda_{2}$. (Recall that $\Lambda\left(\mathcal{C}_{g}\right)$ was defined in (194).) Let both $\mathcal{C}_{g} \in \operatorname{Conf} g\left(\Lambda, \Lambda_{1}\right)$ and $\widetilde{\mathcal{C}}_{g} \in \operatorname{Conf} g\left(\widetilde{\Lambda}, \Lambda_{1}\right)$ lie in $\Lambda \cap \widetilde{\Lambda}$. If further $\mathcal{C}_{g}$ and $\widetilde{\mathcal{C}}_{g}$ have the same gum tree with the same linear order and if they have the same $r$-strips then we say that $\mathcal{C}_{g}$ is equivalent to $\widetilde{\mathcal{C}}_{g}$. Then we have defined an equivalence relation and further, for $\mathcal{C}_{g}$ equivalent to $\widetilde{\mathcal{C}_{g}}$, we have:

$$
\begin{aligned}
\operatorname{Simplex}\left(\mathcal{C}_{g}, T\right) & =\operatorname{Simplex}\left(\widetilde{\mathcal{C}}_{g}, T\right) \quad \text { for all } T \in(0, \infty], \\
\pi_{\Lambda_{1}} \circ \mathcal{L}_{\mathcal{C}_{g}, \mathbf{t}}^{T} \circ \pi_{\Lambda} & =\pi_{\Lambda_{1}} \circ \mathcal{L}_{\widetilde{\mathcal{C}}_{g}, \mathbf{t}}^{T} \circ \pi_{\widetilde{\Lambda}} \quad \text { for all } \mathbf{t} \in \operatorname{Simplex}\left(\mathcal{C}_{g}, T\right) \\
\text { and } \quad \pi_{\Lambda_{1}} \circ \mathcal{L}_{\mathcal{C}_{g}}^{T} \circ \pi_{\Lambda} & =\pi_{\Lambda_{1}} \circ \mathcal{L}_{\widetilde{\mathcal{C}_{g}}}^{T} \circ \pi_{\widetilde{\Lambda}} .
\end{aligned}
$$

(227) and (228) say that the operators in $L\left(\mathcal{H}_{\vartheta}, \mathcal{H}_{\Lambda_{1}}\right)$ are the same. We define by $\operatorname{Conf} g\left(\mathbb{Z}^{d}, \Lambda_{1}\right)$ the set of equivalence classes. Because of (226) and (227) the simplices and operators for each equivalent class can be defined as being equal to the corresponding object for any representative.

We will write $\pi_{\Lambda_{1}} \circ \mathcal{L}_{\mathcal{C}_{g}, \mathrm{t}}^{T}$ instead of $\pi_{\Lambda_{1}} \circ \mathcal{L}_{\mathcal{C}_{g}, \mathrm{t}}^{T} \circ \pi_{\Lambda}$ and $\pi_{\Lambda_{1}} \circ \mathcal{L}_{\mathcal{C}_{g}}^{T}$ instead of $\pi_{\Lambda_{1}} \circ \mathcal{L}_{\mathcal{C}_{g}}^{T} \circ \pi_{\Lambda}$ for the operators from $\mathcal{H}_{\vartheta}$ to $\mathcal{H}_{\Lambda_{1}}$. 
THEOREM 3.25.-

1. There are $0<\tilde{\vartheta}<\vartheta<1$ such that for sufficiently small $\varepsilon$, large $c_{g}$ and every $T \in[0, \infty]$ we can define an operator $\mathcal{L}_{S}^{T}$ from $\mathcal{H}_{\vartheta}$ to $\mathcal{H}_{\tilde{\vartheta}}$ by

$$
\pi_{\Lambda_{1}} \circ \mathcal{L}_{S}^{T} \phi=\sum_{\mathcal{C}_{g} \in \operatorname{Conf}_{g}\left(\mathbb{Z}^{d}, \Lambda_{1}\right)} \pi_{\Lambda_{1}} \circ \mathcal{L}_{\mathcal{C}_{g}}^{T} \phi .
$$

There is a $T_{0}>0$ such that for $T \geqslant T_{0}$ the operator $\mathcal{L}_{S}^{T}$ maps $\mathcal{H}_{\vartheta}$ into $\mathcal{H}_{\vartheta}$. $\mathcal{L}_{S}^{T}$ is the transfer operator, restricted to $\mathcal{H}_{\vartheta}^{b v}$, for the kernel $K_{S}^{T}$, i.e.

$$
\int_{M} \mathrm{~d} \mu\left(K_{S}^{T} \psi\right) \phi=\int_{M} \mathrm{~d} \mu \psi\left(\mathcal{L}_{S}^{T} \phi\right)
$$

for all $\psi \in \mathcal{C}^{0}(M)$ and $\phi \in \mathcal{H}_{\vartheta}^{b v}$.

2. The family $\left(\mathcal{L}_{S}^{T}\right)_{T \geqslant 0}$ in $L\left(\mathcal{H}_{\vartheta}\right)$ converges exponentially fast to $\mathcal{L}_{S}^{\infty}$ :

$$
\left\|\mathcal{L}_{S}^{\infty}-\mathcal{L}_{S}^{T}\right\|_{L\left(\mathcal{H}_{\vartheta}, \mathcal{H}_{\tilde{\vartheta}}\right)} \leqslant c_{5} \mathrm{e}^{-c_{6} T}
$$

for some positive constants $c_{5}, c_{6}$. For sufficiently large $T$ estimate (231) holds also in the norm of $L\left(\mathcal{H}_{\vartheta}\right)$. So among the probability measures corresponding to elements in $\mathcal{H}_{\vartheta}$ there is a unique $K_{S}^{T}$-invariant probability measure $v^{*}$ on $M$, say corresponding to $v \in \mathcal{H}_{\vartheta}$. The operator $\mathcal{L}_{S}^{\infty}$ is a projection onto span $v$ :

$$
\mathcal{L}_{S}^{\infty} \phi=\mu(\phi) \nu .
$$

Proof. - The infinite sum on the rhs of (229) converges as the prove of estimate (204) applies literally to the case $\Lambda=\mathbb{Z}^{d}$. Next we want to show that $\pi_{\Lambda_{1}} \circ \mathcal{L}_{S}^{T}$ is the limit of $\pi_{\Lambda_{1}} \circ \mathcal{L}_{S, \Lambda}^{T}\left(\right.$ as $\left.\Lambda \rightarrow \mathbb{Z}^{d}\right)$. The difference between these two operators is due to configurations $\mathcal{C}_{g}$ in $\operatorname{Conf} g\left(\Lambda, \Lambda_{1}\right)$ or in $\operatorname{Conf} g\left(\mathbb{Z}^{d}, \Lambda_{1}\right)$ with $\Lambda\left(\mathcal{C}_{g}\right) \not \subset \Lambda$. For these we can split in estimate (205) the factor that arises from the decay of interaction in the following way (which is the same as the splitting (110) in [13]).

$$
\exp \left(-c_{g} \sum_{k=1}^{\infty} k^{d} n_{\beta, k}\right) \leqslant \exp \left(-\tilde{c}_{g} \sum_{k=1}^{\infty} k^{d} n_{\beta, k}\right) \exp \left(-\xi \operatorname{dist}\left(\Lambda_{1}, \Lambda^{C}\right)\right)
$$

with a suitably chosen $\xi>0$ such that $\tilde{c}_{g}=c_{g}-\xi>0$. (Note that we can choose $\xi$ so small that the estimates, formerly done with $c_{g}$ work with $\tilde{c}_{g}$ instead as well.) So we can estimate

$$
\begin{aligned}
& \tilde{\vartheta}^{\left|\Lambda_{1}\right|}\left\|\pi_{\Lambda_{1}} \circ \mathcal{L}_{S, \Lambda}^{T}-\pi_{\Lambda_{1}} \circ \mathcal{L}_{S}^{T}\right\|_{L\left(\mathcal{H}_{\vartheta}, \mathcal{H}_{\Lambda_{1}}\right)} \\
& \quad \leqslant 2 \sum_{\substack{\mathcal{C}_{g} \in \operatorname{Conf}\left(\mathbb{Z}^{d}, \Lambda_{1}\right) \\
\Lambda\left(\mathcal{C}_{g}\right) \not \Lambda}} \tilde{\vartheta}^{\left|\Lambda_{1}\right|}\left\|\pi_{\Lambda_{1}} \circ \mathcal{L}_{\mathcal{C}_{g}}^{T}\right\|_{L\left(\mathcal{H}_{\vartheta}, \mathcal{H}_{\Lambda_{1}}\right)} \\
& \leqslant c_{7} \exp \left(-\xi \operatorname{dist}\left(\Lambda_{1}, \Lambda^{C}\right)\right) .
\end{aligned}
$$

Next we show (230) for the special case that $\psi$ depends only on the $\Lambda_{1}$-coordinates, using (213): 


$$
\begin{aligned}
\int_{M} \mathrm{~d} \mu(\mathbf{z})\left(K_{S}^{T} \psi\right)(\mathbf{z}) \phi(\mathbf{z}) & =\lim _{\Lambda \rightarrow \mathbb{Z}^{d}} \int_{M} \mathrm{~d} \mu^{\Lambda}\left(\mathbf{z}_{\Lambda}\right)\left(K_{S, \Lambda}^{T} \psi\right)\left(\mathbf{z}_{\Lambda}\right) \phi_{\Lambda}\left(\mathbf{z}_{\Lambda}\right) \\
& =\lim _{\Lambda \rightarrow \mathbb{Z}^{d}} \int_{\left(S^{1}\right)^{\Lambda}} \mathrm{d} \mu^{\Lambda}\left(\mathbf{z}_{\Lambda}\right) \psi\left(\mathbf{z}_{\Lambda}\right)\left(\mathcal{L}_{S, \Lambda}^{T} \phi_{\Lambda}\right)\left(\mathbf{z}_{\Lambda}\right) \\
& =\lim _{\Lambda \rightarrow \mathbb{Z}^{d}} \int_{\left(S^{1}\right)^{\Lambda_{1}}} \mathrm{~d} \mu^{\Lambda_{1}}\left(\mathbf{z}_{\Lambda}\right) \psi\left(\mathbf{z}_{\Lambda_{1}}\right)\left(\pi_{\Lambda_{1}} \circ \mathcal{L}_{s}^{T} \phi_{\Lambda}\right)\left(\mathbf{z}_{\Lambda_{1}}\right) \\
& =\int_{M} \mathrm{~d} \mu(\mathbf{z}) \psi(\mathbf{z})\left(\mathcal{L}_{S}^{T} \phi\right)(\mathbf{z}) .
\end{aligned}
$$

We conclude (230) for general $\psi \in \mathcal{C}^{0}(M)$ by approximating it by $\psi_{\Lambda_{1}}$ (cf. (141)), depending only on the $\Lambda_{1}$-coordinates and using continuity w.r.t. $\psi$ of both sides of (230). So 1. is proved.

Next we show (231). We note that for $\Lambda_{1}=\emptyset$ the lhs (236) in the following estimate is equal to zero as both transfer operators preserve the Lebesgue integral ( $\mu$ is a 'left eigenvector' with eigenvalue 1.) So we only have to consider the case $\left|\Lambda_{1}\right| \geqslant 1$.

$$
\begin{aligned}
& \tilde{\vartheta}^{\left|\Lambda_{1}\right|}\left\|\pi_{\Lambda_{1}} \circ \mathcal{L}_{S}^{\infty}-\pi_{\Lambda_{1}} \circ \mathcal{L}_{S}^{T}\right\|_{L\left(\mathcal{H}_{\vartheta}, \mathcal{H}_{\Lambda_{1}}\right)} \\
& \leqslant \tilde{\vartheta}^{\left|\Lambda_{1}\right|} \sum_{\mathcal{C}_{g} \in \operatorname{Conf}\left(\mathbb{Z}^{d}, \Lambda_{1}\right)}\left\|\pi_{\Lambda_{1}} \circ \mathcal{L}_{\mathcal{C}_{g}}^{\infty}-\pi_{\Lambda_{1}} \circ \mathcal{L}_{\mathcal{C}_{g}}^{T}\right\|_{L\left(\mathcal{H}_{\vartheta}, \mathcal{H}_{\Lambda_{1}}\right)} \\
& \leqslant \tilde{\vartheta}^{\left|\Lambda_{1}\right|}\left\|\mathcal{Q}_{\Lambda_{1}} \circ \pi_{\Lambda_{1}}-\left(1-\mathrm{e}^{-\lambda T}\right)^{\left|\Lambda_{1}\right|} \mathcal{Q}_{\Lambda_{1}} \circ \pi_{\Lambda_{1}}\right\|_{L\left(\mathcal{H}_{\vartheta}, \mathcal{H}_{\Lambda_{1}}\right)} \\
& +\tilde{\vartheta}^{\left|\Lambda_{1}\right|} \sum_{\substack{\mathcal{C}_{g} \in \operatorname{Confg}\left(\mathbb{Z}^{d}, \Lambda_{1}\right), \mathcal{C}_{g} \text { reaches the top }}}\left\|\pi_{\Lambda_{1}} \circ \mathcal{L}_{\mathcal{C}_{g}}^{T}\right\|_{L\left(\mathcal{H}_{\vartheta}, \mathcal{H}_{\Lambda_{1}}\right)} \\
& +\tilde{\vartheta}^{\left|\Lambda_{1}\right|} \sum_{\substack{\mathcal{C}_{g} \in \operatorname{Confg}\left(\mathbb{Z}^{d}, \Lambda_{1}\right), \\
\text { cos } \operatorname{Simplex}\left(\mathcal{C}_{g}, \frac{T}{2}\right) \\
\text { reach the top, } \\
\left|n_{\beta}\left(\mathcal{C}_{g}\right)\right| \geqslant 1}} \lambda^{\left|n_{\beta}\left(\mathcal{C}_{g}\right)\right|} \mathrm{dt} \\
& \times\left\|\pi_{\Lambda_{1}} \circ \mathcal{L}_{\mathcal{C}_{g}, \mathbf{t}}^{\infty}-\pi_{\Lambda_{1}} \circ \mathcal{L}_{\mathcal{C}_{g}, \mathbf{t}}^{T}\right\|_{L\left(\mathcal{H}_{\vartheta}, \mathcal{H}_{\Lambda_{1}}\right)} \\
& +\tilde{\vartheta}^{\left|\Lambda_{1}\right|} \sum_{\mathcal{C}_{g} \in \operatorname{Confg}\left(\mathbb{Z}^{d}, \Lambda_{1}\right),} \int_{\operatorname{Simplex}\left(\mathcal{C}_{g}, \infty\right)} \lambda^{\left|n_{\beta}\left(\mathcal{C}_{g}\right)\right|} \mathrm{dt} \\
& \begin{array}{c}
\mathcal{C}_{g} \text { does not reach the top, } \backslash \operatorname{Simplex}\left(\mathcal{C}_{g}, \frac{T}{2}\right) \\
\qquad\left|n_{\beta}\left(\mathcal{C}_{g}\right)\right| \geqslant 1
\end{array} \\
& \times\left\|\pi_{\Lambda_{1}} \circ \mathcal{L}_{\mathcal{C}_{g}, \mathbf{t}}^{\infty}\right\|_{L\left(\mathcal{H}_{\vartheta}, \mathcal{H}_{\Lambda_{1}}\right)} \\
& +\tilde{\vartheta}^{\left|\Lambda_{1}\right|} \sum_{\mathcal{C}_{g} \in \operatorname{Confg}\left(\mathbb{Z}^{d}, \Lambda_{1}\right),} \int_{\operatorname{Simplex}\left(\mathcal{C}_{g}, T\right)} \lambda^{\left|n_{\beta}\left(\mathcal{C}_{g}\right)\right|} \mathrm{d} \mathbf{t} \\
& \begin{array}{c}
\mathcal{C}_{g} \text { does not reach the top, } \backslash \operatorname{Simplex}\left(\mathcal{C}_{g}, \frac{T}{2}\right) \\
\qquad\left|n_{\beta}\left(\mathcal{C}_{g}\right)\right| \geqslant 1
\end{array} \\
& \times\left\|\pi_{\Lambda_{1}} \circ \mathcal{L}_{\mathcal{C}_{g}, \mathbf{t}}^{T}\right\|_{L\left(\mathcal{H}_{\vartheta}, \mathcal{H}_{\Lambda_{1}}\right)} .
\end{aligned}
$$


We have distinguished between the following classes of gum configurations. The first summand (237) corresponds to the operator $\pi_{\Lambda_{1}} \circ \mathcal{L}_{\mathcal{C}_{g}}^{\infty}-\pi_{\Lambda_{1}} \circ \mathcal{L}_{\mathcal{C}_{g}}^{T}$ where $\mathcal{C}_{g}$ is the gum configuration that has only long $h$-strips (no branchings or $r$-strips). The second summand (238) takes all $\mathcal{C}_{g}$ into account that reach the top. So all specified configurations $\left(\mathcal{C}_{g}, T, \mathbf{t}\right)$ have an $r$-strip ending at time $-T$. All $\left(\mathcal{C}_{g}, \infty, \mathbf{t}\right)$ have an infinitely long $r$-strip and so the corresponding operator is zero (cf. Remark 3.21.3) and does not appear in (238). The last three summands, (239), (240) and (241), correspond to $\mathcal{C}_{g}$ that do not reach the top and do not consist only of $h$-strips. That implies that it has at least one branching and the corresponding domains of integration, $\operatorname{Simplex}\left(\mathcal{C}_{g}, \infty\right)$ and $\operatorname{Simplex}\left(\mathcal{C}_{g}, T\right)$, are not degenerated to a point. We divide them into $\operatorname{Simplex}\left(\mathcal{C}_{g}, \frac{T}{2}\right)$ and the particular complements. The reason for this will become clear when we do the estimates. In (239) we integrate the norm of the operator difference $\pi_{\Lambda_{1}} \circ \mathcal{L}_{\mathcal{C}_{g}}^{\infty}-\pi_{\Lambda_{1}} \circ \mathcal{L}_{\mathcal{C}_{g}}^{T}$ over Simplex $\left(\mathcal{C}_{g}, \frac{T}{2}\right)$ and in (240) and (241) we integrate the norms of the two operators separately over the particular complement sets.

Now we estimate each summand: The first summand (237) is estimated by

$$
\begin{aligned}
\tilde{\vartheta}^{\left|\Lambda_{1}\right|} c_{h}^{\left|\Lambda_{1}\right|}\left(1-\left(1-\mathrm{e}^{-\lambda T}\right)^{\left|\Lambda_{1}\right|}\right) & \leqslant\left(\tilde{\vartheta} c_{h}\right)^{\left|\Lambda_{1}\right|} \sum_{k=1}^{\left|\Lambda_{1}\right|}\left(\begin{array}{c}
\left|\Lambda_{1}\right| \\
k
\end{array}\right)\left(\mathrm{e}^{-\lambda T}\right)^{k} \\
& \leqslant\left(\tilde{\vartheta} c_{h}\left(1+\mathrm{e}^{-\frac{1}{2} \lambda T}\right)\right)^{\left|\Lambda_{1}\right|} \mathrm{e}^{-\frac{1}{2} \lambda T} \leqslant \mathrm{e}^{-\frac{1}{2} \lambda T},
\end{aligned}
$$

where the last inequality holds if $\tilde{\vartheta}$ is chosen sufficiently small.

For estimating the last summand (241) we note that for $\mathbf{t} \in \operatorname{Simplex}\left(\mathcal{C}_{g}, T\right) \backslash$ $\operatorname{Simplex}\left(\mathcal{C}_{g}, \frac{T}{2}\right)$ the sum of the lengths of all $r$-strips of $\left(\mathcal{C}_{g}, T, \mathbf{t}\right)$ is at least $\frac{T}{2}$. (This is because $\left(\mathcal{C}_{g}, T, \mathbf{t}\right)$ has a branching, say at time $t_{i}$ with $\left|t_{i}\right| \geqslant \frac{T}{2}$ and there must be a sequence of apex-r-strips whose lengths add up to at least $\frac{T}{2}$.) So if we split in the estimate (170) for each maximal $r$-chain, of length $t$ say, the rhs

$$
\|\mathcal{R}(t)\| \leqslant c_{r} \mathrm{e}^{-(1-\eta) \lambda t}=c_{r} \mathrm{e}^{-\frac{1-\eta}{2} \lambda t} \mathrm{e}^{-\frac{1-\eta}{2} \lambda t}
$$

we can extract the second factor $\exp \left(-\frac{1-\eta}{2} \lambda t\right)$. Their product is bounded from above by $\exp \left(-\frac{1-\eta}{2} \lambda \frac{T}{2}\right)$. We assume that the coupling parameters $\varepsilon$ (small) and $c_{g}$ (large) are such that our analysis still holds with the (in the sum) remaining factors $\exp \left(-\frac{1-\eta}{2} \lambda\right.$ length $\left.(R)\right)$ for each maximal $r$-chain $R$.

We get

$$
\begin{gathered}
\tilde{\vartheta}^{\left|\Lambda_{1}\right|} \sum_{\substack{\mathcal{C}_{g} \in \operatorname{Confg}\left(\mathbb{Z}^{d}, \Lambda_{1}\right), \mathcal{C}_{g} \text { does not reach the top, } \\
\left|n_{\beta}\left(\mathcal{C}_{g}\right)\right| \geqslant 1}} \int_{\substack{\operatorname{Simplex}\left(\mathcal{C}_{g}, T\right) \\
\mid \operatorname{Simplex}\left(\mathcal{C}_{g}, \frac{T}{2}\right)}} \lambda^{\left|n_{\beta}\left(\mathcal{C}_{g}\right)\right|} \mathrm{d} \mathbf{t}\left\|\pi_{\Lambda_{1}} \circ \mathcal{L}_{\mathcal{C}_{g}, \mathbf{t}}^{T}\right\|_{L\left(\mathcal{H}_{\vartheta}, \mathcal{H}_{\Lambda_{1}}\right)} \\
\leqslant c_{8} \exp \left(-\lambda \frac{1-\eta}{2} \frac{T}{2}\right) .
\end{gathered}
$$

Similarly, we can estimate the second (238) and the fourth (240) summand:

$$
\tilde{\vartheta}^{\left|\Lambda_{1}\right|} \sum_{\substack{\mathcal{C}_{g} \in \operatorname{Conf}\left(\mathbb{Z}^{d}, \Lambda_{1}\right), \mathcal{C}_{g} \text { reaches the top }}}\left\|\pi_{\Lambda_{1}} \circ \mathcal{L}_{\mathcal{C}_{g}}^{T}\right\|_{L\left(\mathcal{H}_{\vartheta}, \mathcal{H}_{\Lambda_{1}}\right)} \leqslant c_{9} \exp \left(-\frac{1-\eta}{2} \lambda T\right),
$$




$$
\begin{aligned}
& \tilde{\vartheta}^{\left|\Lambda_{1}\right|} \sum_{\mathcal{C}_{g} \in \operatorname{Conf}\left(\mathbb{Z}^{d}, \Lambda_{1}\right)} \int_{\substack{\operatorname{Simplex}\left(\mathcal{C}_{g}, \infty\right) \\
\mid \operatorname{Simplex}\left(\mathcal{C}_{g}, \frac{T}{2}\right)}} \lambda^{\left|n_{\beta}\left(\mathcal{C}_{g}\right)\right|} \mathrm{d} \mathbf{t}\left\|\pi_{\Lambda_{1}} \circ \mathcal{L}_{\mathcal{C}_{g}, \boldsymbol{t}}^{\infty}\right\|_{L\left(\mathcal{H}_{\vartheta}, \mathcal{H}_{\Lambda_{1}}\right)} \\
& \leqslant c_{10} \exp \left(-\frac{1-\eta}{2} \lambda \frac{T}{2}\right) .
\end{aligned}
$$

We estimate the third summand (239) we use Lemma 3.26 (see below). For that we note that for $\mathcal{C}_{g} \in \operatorname{Conf} g\left(\mathbb{Z}^{d}, \Lambda_{1}\right)$ and $\mathbf{t} \in \operatorname{Simplex}\left(\mathcal{C}_{g}, \frac{T}{2}\right)$ the operators $\pi_{\Lambda_{1}} \circ \mathcal{L}_{\mathcal{C}_{g}, \mathbf{t}}^{\infty}$ and $\pi_{\Lambda_{1}} \circ \mathcal{L}_{\mathcal{C}_{g}, \mathbf{t}}^{T}$ can both be written as a product of numbers $\left(1-\mathrm{e}^{- \text {length }(H)}\right)$ and operators $\mathcal{Q}_{q} \otimes \mathrm{id}_{\Lambda \backslash\{q\}}$, corresponding to maximal $h$-strips, $\mathcal{R}_{q} \otimes \mathrm{id}_{\Lambda \backslash\{q\}}$, corresponding to maximal $r$-strips, and $\mathcal{B}_{q} \otimes \operatorname{id}_{\Lambda \backslash\{q\}}$, corresponding to branchings. They have the same structure in the sense that these factors are in 1-1-correspondence and the quantitative difference is only due to $h$-strips going to the top or long $h$-strips in $\Lambda_{1}$ as we can see from representation (199) for $\mathcal{L}_{\mathcal{C}_{g}, \mathbf{t}}^{\infty}$ and $\mathcal{L}_{\mathcal{C}_{g}, \mathbf{t}}^{T}$ and also from Remark 3.21.1. So they differ only in the constants $\tilde{c}\left(\mathcal{C}_{g}, \infty, \mathbf{t}\right)$ and $\tilde{c}\left(\mathcal{C}_{g}, T, \mathbf{t}\right)$. More precisely, an $h$-strip in $\mathcal{C}_{g}$ that goes to the top and therefore corresponds to an $h$-strip in $\left(\mathcal{C}_{g}, T, \mathbf{t}\right)$, say from $\left(q, t_{i}\right)$ to $(q,-T)$, and so gives rise to a factor $1-\exp \left(-\lambda\left(T-\left|t_{i}\right|\right)\right)\left(\right.$ note that $\left.\left|t_{i}\right|<\frac{T}{2}\right)$ whilst the corresponding $h$-strip in $\left(\mathcal{C}_{g}, \infty, \mathbf{t}\right)$ ends at time $-\infty$ and gives rise to a factor 1. Similarly a long $h$-strip of $\mathcal{C}_{g}$ in $\Lambda_{1}$ gives rise to factors $1-\exp (-\lambda T)$ and 1 , respectively. In both cases the difference between the scalar factors (for each $h$-strip to the top) is bounded by

$$
\delta^{2}=\exp \left(-\frac{\lambda}{2} T\right)
$$

The number of $h$-strips to the top is bounded by $\sum_{k=1}^{\infty} 3^{d} n_{\beta, k} k^{d}$ and the number of long $h$-strips at sites in $\Lambda_{1}$ by $\left|\Lambda_{1}\right|-K$ (where $n_{\beta, k}$ and $K$ are the parameters of the labelled tree of $\mathcal{C}_{g}$.)

So we estimate

$$
\begin{aligned}
& \tilde{\vartheta}^{\left|\Lambda_{1}\right|}\left\|\pi_{\Lambda_{1}} \circ \mathcal{L}_{\mathcal{C}_{g}, \mathbf{t}}^{\infty} \phi-\pi_{\Lambda_{1}} \circ \mathcal{L}_{\mathcal{C}_{g}, \mathbf{t}}^{T} \phi\right\|_{\Lambda_{1}} \\
& \leqslant \delta \prod_{k=1}^{\infty}(1+\delta)^{3^{d} n_{\beta, k} k^{d}}(1+\delta)^{\left|\Lambda_{1}\right|-K}\left(c_{3} \varepsilon\right)^{\left|n_{\beta}\right|} \exp \left(-c_{g} \sum_{k=1}^{\infty} k^{d} n_{\beta, k}\right) c_{h}^{\tilde{n}_{h}} c_{r}^{\tilde{n}_{r}} \\
& \quad \times \prod_{R} \exp (-(1-\eta) \lambda \operatorname{length}(R)) \tilde{c}\left(\mathcal{C}_{g}, T, \mathbf{t}\right)\left\|\phi_{\Lambda\left(\mathcal{C}_{g}\right)}\right\|_{\Lambda\left(\mathcal{C}_{g}\right)} .
\end{aligned}
$$

The factor $(1+\delta)^{3^{d} n_{\beta, k} k^{d}}$ and the factor $\vartheta^{-(3 k)^{d}} n_{\beta, k}$ that we get from the estimate (206) of $\left\|\phi_{\Lambda\left(\mathcal{C}_{g}\right)}\right\|_{\Lambda\left(\mathcal{C}_{g}\right)}$ are compensated for by $\exp \left(-c_{g} k^{d} n_{\beta, k}\right)$ 'in the usual way'. If $\varepsilon$ is sufficiently small and $c_{g}$ large we can estimate

$$
\begin{gathered}
\mathcal{C}_{g} \in \operatorname{Conf}\left(\mathbb{Z}^{d}, \Lambda_{1}\right), \quad \operatorname{Simplex}\left(\mathcal{C}_{g}, \frac{T}{2}\right) \\
\mathcal{C}_{g} \text { does not reach the top, } \\
\\
\left|n_{\beta}\left(\mathcal{C}_{g}\right)\right| \geqslant 1 \\
\leqslant c_{11} \mathrm{e}^{-\frac{\lambda}{4} T} .
\end{gathered}
$$


From (242), (245), (249), (246), and (244) we conclude (231) with $c_{6}=\frac{1-\eta}{4} \lambda$ and $c_{5}$ sufficiently large.

For any $\phi \in \mathcal{H}_{\vartheta}$ and any $\Lambda \in \mathcal{F}$ we have

$$
\begin{aligned}
\pi_{\Lambda} \circ \mathcal{L}_{S}^{\infty} \phi=\sum_{\substack{\mathcal{C}_{g} \in \operatorname{Confg}\left(\mathbb{Z}^{d}, \Lambda\right), \mathcal{C}_{g} \text { does not reach the top }}} \pi_{\Lambda} \circ \mathcal{L}_{\mathcal{C}_{g}}^{\infty} \phi \\
=\sum_{\substack{\mathcal{C}_{g} \in \operatorname{Confg}\left(\mathbb{Z}^{d}, \Lambda\right), \mathcal{C}_{g} \text { does not reach the top }}}\left(\pi_{\Lambda} \circ \mathcal{L}_{\mathcal{C}_{g}}^{\infty} h_{\mathbb{Z}^{d}}\right) \cdot \mu(\phi) .
\end{aligned}
$$

The sum in (250) is a priori over all $\mathcal{C}_{g} \in \operatorname{Conf}_{g}\left(\mathbb{Z}^{d}, \Lambda\right)$ but, as we have seen before, if $\mathcal{C}_{g}$ reaches the top the corresponding operator $\pi_{\Lambda} \circ \mathcal{L}_{\mathcal{C}_{g}}^{\infty}$ is zero. If $\mathcal{C}_{g}$ does not reach the top there are only $h$-strips going to the top $(-\infty)$ and $\pi_{\Lambda} \circ \mathcal{L}_{\mathcal{C}_{g}}^{\infty}$ is a projection onto $\operatorname{span}\left(h_{\mathbb{Z}^{d}}\right)$.

We set $v_{\Lambda} \stackrel{\text { def }}{=} \pi_{\Lambda} \circ \mathcal{L}_{\mathcal{C}_{g}}^{\infty} h_{\mathbb{Z}^{d}}$ and this defines $v=\left(v_{\Lambda}\right)_{\Lambda \in \mathcal{F}}$. Note that the transfer operator $\mathcal{L}_{S}^{\infty}$ preserves the Lebesgue integral and so $v_{\emptyset}=1$, i.e. $v$ corresponds to a probability measure.

In the proof of Theorem 3.25 we have used the following lemma.

LEMMA 3.26. - Let $A_{1}, \ldots, A_{n}, \tilde{A}_{1}, \ldots, \tilde{A}_{n}$ be operators on the same Banach space, $0<\delta<1$ and $a_{1}, \ldots, a_{n}$ positive numbers such that:

$$
\begin{aligned}
\left\|A_{i}\right\| & \leqslant a_{i} \quad \text { for all } 1 \leqslant i \leqslant n \\
\text { and }\left\|A_{i}-\tilde{A}_{i}\right\| & \leqslant \delta^{2} a_{i} .
\end{aligned}
$$

Then

$$
\left\|A_{1} \circ \cdots \circ A_{n}-\tilde{A}_{1} \circ \cdots \circ \tilde{A}_{n}\right\| \leqslant \delta(1+\delta)^{n} a_{1} \cdots a_{n} .
$$

Proof. - From (252) we get

$$
\left\|\tilde{A}_{i}\right\| \leqslant\left(1+\delta^{2}\right) a_{i}
$$

So we get via 'telescope expansion':

$$
\begin{aligned}
& \left\|A_{1} \circ \cdots \circ A_{n}-\tilde{A}_{1} \circ \cdots \circ \tilde{A}_{n}\right\| \\
& \leqslant\left\|A_{1} \circ \cdots \circ A_{n}-\tilde{A}_{1} \circ A_{2} \circ \cdots \circ A_{n}\right\|+\cdots \\
& +\left\|\tilde{A}_{1} \circ \cdots \circ \tilde{A}_{n-1} \circ A_{n}-\tilde{A}_{1} \circ \cdots \circ \tilde{A}_{n}\right\| \\
& \leqslant \delta^{2}\left(1+\left(1+\delta^{2}\right)+\cdots+\left(1+\delta^{2}\right)^{n-1}\right) a_{1} \cdots a_{n} \\
& =\left(\left(1+\delta^{2}\right)^{n}-1\right) a_{1} \cdots a_{n} \\
& =\sum_{k=1}^{n}\left(\begin{array}{l}
n \\
k
\end{array}\right) \delta^{2 k} a_{1} \cdots a_{n} \leqslant \delta \sum_{k=1}^{n}\left(\begin{array}{l}
n \\
k
\end{array}\right) \delta^{k} a_{1} \cdots a_{n} \\
& \leqslant \delta(1+\delta)^{n} a_{1} \cdots a_{n}
\end{aligned}
$$

and the lemma is proved. 
Remark 3.27. - Analogously to Proposition 6.1.3 in [13], one can also prove a semigroup-like property of the family $\left(\mathcal{L}_{S}^{t}\right)_{t \geqslant 0}$, using Remark 2.17 and the diagrammatic representation of the operators.

\section{Decay of correlations}

In the following theorem which is completely analogous to Theorem 7.1 in [13], we state the mixing properties for the invariant probability measure $v^{*}$ in terms of the weighted norms.

THEOREM 4.1. - For sufficiently small $\vartheta, \tilde{\vartheta}, \varepsilon$ and big $c_{g}$ there is $a \kappa \in(0,1)$ and positive constants $c_{12}, c_{13}, c_{14}$ and $c_{15}$ such that for all finite disjoint $\Lambda_{1}, \Lambda_{2} \subset \mathbb{Z}^{d}$ and $\psi \in \mathcal{H}_{\Lambda_{2}}$ the following holds:

$$
\begin{aligned}
& \left\|v_{\Lambda_{1} \cup \Lambda_{2}}-v_{\Lambda_{1}} v_{\Lambda_{2}}\right\|_{\Lambda_{1} \cup \Lambda_{2}} \leqslant c_{12} \vartheta^{-\left|\Lambda_{1} \cup \Lambda_{2}\right|} \kappa^{\operatorname{dist}\left(\Lambda_{1}, \Lambda_{2}\right)}, \\
& \left\|\pi_{\Lambda_{1}}(\psi v)-v^{*}(\psi) v_{\Lambda_{1}}\right\|_{\Lambda_{1}} \leqslant c_{13} \vartheta^{-\left|\Lambda_{1} \cup \Lambda_{2}\right|}\|\psi\|_{\Lambda_{2}} \kappa^{\operatorname{dist}\left(\Lambda_{1}, \Lambda_{2}\right)}, \\
& \left\|\pi_{\Lambda_{1}} \circ \mathcal{L}_{S}^{T}(\psi v)-v^{*}(\psi) v_{\Lambda_{1}}\right\|_{\Lambda_{1}} \leqslant c_{14} \vartheta^{-\left|\Lambda_{2}\right|} \tilde{\vartheta}^{-\left|\Lambda_{1}\right|}\|\psi\|_{\Lambda_{2}} \kappa^{\operatorname{dist}\left(\Lambda_{1}, \Lambda_{2}\right)} \\
& \times \exp \left(-c_{15} T\right)
\end{aligned}
$$

for every $T>0$.

Proof. - For a gum configuration $\mathcal{C}_{g}$ we define in analogy to (109) in [13]

$$
b\left(\mathcal{C}_{g}\right) \stackrel{\text { def }}{=} \sum_{k=1}^{\infty} k n_{\beta, k}\left(\mathcal{C}_{g}\right) .
$$

In the following we split gum configurations $\mathcal{C}_{g} \in \operatorname{Conf}\left(\mathbb{Z}^{d}, \Lambda_{1} \cup \Lambda_{2}\right)$ with $b\left(\mathcal{C}_{g}\right) \leqslant$ $\frac{1}{2} \operatorname{dist}\left(\Lambda_{1}, \Lambda_{2}\right)$ into $\mathcal{C}_{g}=\mathcal{C}_{g}^{1} \cup \mathcal{C}_{g}^{2}$ with $\mathcal{C}_{g}^{1} \in \operatorname{Conf}\left(\mathbb{Z}^{d}, \Lambda_{1}\right), \mathcal{C}_{g}^{2} \in \operatorname{Conf}\left(\mathbb{Z}^{d}, \Lambda_{2}\right)$ and $\Lambda\left(\mathcal{C}_{g}^{1}\right) \cap \Lambda\left(\mathcal{C}_{g}^{2}\right)=\emptyset$.

We write, using (232) and the notation of (158):

$$
\begin{aligned}
v_{\Lambda_{1} \cup \Lambda_{2}}= & \sum_{\substack{\mathcal{C}_{g}=\mathcal{C}_{g}^{1} \cup \mathcal{C}_{g}^{2} \in \operatorname{Conf}\left(\mathbb{Z}^{d}, \Lambda_{1} \cup \Lambda_{2}\right) \\
b\left(\mathcal{C}_{g}\right) \leqslant \frac{1}{2} \operatorname{dist}\left(\Lambda_{1}, \Lambda_{2}\right)}}\left(\pi_{\Lambda_{1}} \circ \mathcal{L}_{\mathcal{C}_{g}^{1}}^{T} h_{\mathbb{Z}^{d}}\right)\left(\pi_{\Lambda_{2}} \circ \mathcal{L}_{\mathcal{C}_{g}^{2}}^{T} h_{\mathbb{Z}^{d}}\right) \\
& +\sum_{\substack{\mathcal{C}_{g} \in \operatorname{Confg}\left(\mathbb{Z}^{d}, \Lambda_{1} \cup \Lambda_{2}\right), b\left(\mathcal{C}_{g}\right)>\frac{1}{2} \operatorname{dist}\left(\Lambda_{1}, \Lambda_{2}\right)}} \pi_{\Lambda_{1} \cup \Lambda_{2}} \circ \mathcal{L}_{\mathcal{C}_{g}}^{T} h_{\mathbb{Z}^{d}} .
\end{aligned}
$$

In estimating the norm of the second summand in (260) we can take out from the estimate for $\left\|\pi_{\Lambda_{1}} \circ \mathcal{L}_{\mathcal{C}_{g}}^{\infty} h_{\mathbb{Z}^{d}}\right\|$ a factor

$$
\exp \left(-\xi \frac{1}{2} \operatorname{dist}\left(\Lambda_{1}, \Lambda_{2}\right)\right)=\kappa^{\operatorname{dist}\left(\Lambda_{1}, \Lambda_{2}\right)}
$$


like in (233) such that we get

$$
\left\|\sum_{\substack{\mathcal{C}_{g} \in \operatorname{Conf}\left(\mathbb{Z}^{d}, \Lambda_{1} \cup \Lambda_{2}\right), b\left(\mathcal{C}_{g}\right)>\frac{1}{2} \operatorname{dist}\left(\Lambda_{1}, \Lambda_{2}\right)}} \pi_{\Lambda_{1} \cup \Lambda_{2}} \circ \mathcal{L}_{\mathcal{C}_{g}}^{T} h_{\mathbb{Z}^{d}}\right\| \leqslant c_{16} \vartheta^{\left|\Lambda_{1} \cup \Lambda_{2}\right|} \kappa^{\operatorname{dist}\left(\Lambda_{1}, \Lambda_{2}\right)} .
$$

We write the first summand in (260) as

$$
\begin{aligned}
& \sum_{\substack{\mathcal{C}_{g}=\mathcal{C}_{g}^{1} \cup \mathcal{C}_{g}^{2} \in \operatorname{Conf}\left(\mathbb{Z}^{d}, \Lambda_{1} \cup \Lambda_{2}\right), b\left(\mathcal{C}_{g}\right) \leqslant \frac{1}{2} \operatorname{dist}\left(\Lambda_{1}, \Lambda_{2}\right)}}\left(\pi_{\Lambda_{1}} \circ \mathcal{L}_{\mathcal{C}_{g}^{1}}^{T} h_{\mathbb{Z}^{d}}\right)\left(\pi_{\Lambda_{2}} \circ \mathcal{L}_{\mathcal{C}_{g}^{2}}^{T} h_{\mathbb{Z}^{d}}\right) \\
& =v_{\Lambda_{1}} v_{\Lambda_{2}}-\sum_{\substack{\mathcal{C}_{g}^{1} \operatorname{Confg}\left(\mathbb{Z}^{d}, \Lambda_{1}\right), \mathcal{C}_{g}^{2} \in \operatorname{Confg}\left(\mathbb{Z}^{d}, \Lambda_{2}\right), b\left(\mathcal{C}_{g}^{1}\right)+b\left(\mathcal{C}_{g}^{2}\right)>\frac{1}{2} \operatorname{dist}\left(\Lambda_{1}, \Lambda_{2}\right)}}\left(\pi_{\Lambda_{1}} \circ \mathcal{L}_{\mathcal{C}_{g}^{1}}^{T} h_{\mathbb{Z}^{d}}\right)\left(\pi_{\Lambda_{2}} \circ \mathcal{L}_{\mathcal{C}_{g}^{2}}^{T} h_{\mathbb{Z}^{d}}\right) \\
&
\end{aligned}
$$

and estimate

$$
\left\|\sum_{\substack{\mathcal{C}_{g}^{1} \operatorname{Confg}\left(\mathbb{Z}^{d}, \Lambda_{1}\right), \mathcal{C}_{g}^{2} \in \operatorname{Confg}\left(\mathbb{Z}^{d}, \Lambda_{2}\right), b\left(\mathcal{C}_{g}^{1}\right)+b\left(\mathcal{C}_{g}^{2}\right)>\frac{1}{2} \operatorname{dist}\left(\Lambda_{1}, \Lambda_{2}\right)}}\left(\pi_{\Lambda_{1}} \circ \mathcal{L}_{\mathcal{C}_{g}^{1}}^{T} h_{\mathbb{Z}^{d}}\right)\left(\pi_{\Lambda_{2}} \circ \mathcal{L}_{\mathcal{C}_{g}^{2}}^{T} h_{\mathbb{Z}^{d}}\right)\right\| \leqslant c_{17} \vartheta^{\left|\Lambda_{1} \cup \Lambda_{2}\right|} \kappa^{\operatorname{dist}\left(\Lambda_{1}, \Lambda_{2}\right)} .
$$

From (262), (263) and (264) we conclude (256). The proof of (257), using (256), is the same as in [13].

To prove (258) we set $\phi=\psi v-v(\psi) v$. So

$$
\pi_{\Lambda_{1}} \circ \mathcal{L}_{S}^{T} \phi=\pi_{\Lambda_{1}} \circ \mathcal{L}_{S}^{T}(\psi v)-v(\psi) v_{\Lambda_{1}}
$$

and in particular

$$
\mathcal{L}_{S}^{\infty} \phi=0 .
$$

We estimate (265), analogously to (129) in [13], using the finer estimate

$$
\left\|\phi_{\Lambda(\mathcal{C})}\right\|_{\Lambda(\mathcal{C})} \leqslant c_{13} \vartheta^{-\left|\Lambda_{2}\right|}\|\psi\|_{\Lambda_{2}} \vartheta^{-\left|\Lambda_{r}(\mathcal{C})\right|-\sum_{k=1}^{\infty}(3 k)^{d} n_{\beta, k}} \kappa^{\operatorname{dist}\left(\Lambda_{1}, \Lambda_{2}\right)-\sum_{k=1}^{\infty} k n_{\beta, k}}
$$

that we get from (257). For each $\mathcal{C}_{g}$ we get a 'good' factor $\kappa^{\operatorname{dist}\left(\Lambda_{1}, \Lambda_{2}\right)}$ that we can take out of the sum (over gum configurations), and a 'bad' factor $\kappa^{-\sum_{k=1}^{\infty} k n_{\beta, k}}$. The latter is compensated for in the usual way by the factor $\exp \left(-c_{g} \sum_{k=1}^{\infty} k^{d} n_{\beta, k}\right)$, provided that $c_{g}$ is sufficiently large.

Using (266) and (267), we get with the same argument as for the proof of (231):

$$
\tilde{\vartheta}^{\left|\Lambda_{1}\right|}\left\|\pi_{\Lambda_{1}} \circ \mathcal{L}_{S}^{T} \phi\right\| \leqslant c_{18} \vartheta^{-\left|\Lambda_{2}\right|}\|\psi\| \kappa^{\operatorname{dist}\left(\Lambda_{1}, \Lambda_{2}\right)} \exp \left(-c_{15} T\right)
$$

and (258) follows.

For our last theorem we need some definitions. 
DEFINITION 4.2. - Every $\tau=\left(\tau_{1}, \ldots, \tau_{d}\right) \in \mathbb{Z}^{d}$ defines a shift on lattice $\mathbb{Z}^{d}$ by $\left(\alpha_{1}, \ldots, \alpha_{d}\right)+\left(\tau_{1}, \ldots, \tau_{d}\right)=\left(\alpha_{1}+\tau_{1}, \ldots, \alpha_{d}+\tau_{d}\right)$, and so a shift on $\left(S^{1}\right)^{\mathbb{Z}^{d}}$ by $(\tau(\mathbf{x}))_{\alpha} \stackrel{\text { def }}{=} x_{\alpha+\tau}$ for $\mathbf{x} \in\left(S^{1}\right)^{\mathbb{Z}^{d}}$. The size $m(\tau)$ of the shift $\tau$ is $m(\tau) \stackrel{\text { def }}{=}\left|\tau_{1}\right|+\ldots+\left|\tau_{d}\right|$.

We further define a shift on functions $\psi \in \mathcal{C}\left(\left(S^{1}\right)^{\mathbb{Z}^{d}}\right)$ by $(\psi \circ \tau)(\mathbf{x}) \stackrel{\text { def }}{=} \psi(\tau(\mathbf{x}))$.

The family of maps $\left(f_{q}\right)_{q \in \mathbb{Z}^{d}}$, introduced in (149), is called translation-invariant if these maps are all the same, i.e. $f_{q}=f$ for some $f$ and all $q \in \mathbb{Z}^{d}$.

The family $\left(g_{q, k}\right)_{q \in \mathbb{Z}^{d}}$, introduced in (151), is called translation-invariant if $g_{q, k}(\tau(\mathbf{z}))=g_{q-\tau, k}(\mathbf{z})$ for all $q, \tau \in \mathbb{Z}^{d}$ and $\mathbf{z} \in\left(\left(S^{1}\right)^{\mathbb{Z}^{d}}\right)$.

If $\left(f_{q}\right)_{q \in \mathbb{Z}^{d}}$ and also the families $\left(g_{q, k}\right)_{q \in \mathbb{Z}^{d}}$ are translation-invariant then we say that the system is translation-invariant.

Remark 4.3. - In case of a translation-invariant system we also have translationinvariance of the action of Markov-kernels on functions: $K_{S}^{T}(\psi \circ \tau)=\left(K_{S}^{T}(\psi)\right) \circ \tau$ for all $\psi \in \mathcal{C}\left(\left(S^{1}\right)^{\mathbb{Z}^{d}}\right)$ and $\tau \in \mathbb{Z}^{d}$.

We can state the mixing properties of $v^{*}$ w.r.t. spatio-temporal shifts in terms of correlation functions for observables $\psi_{1}, \psi_{2} \in \mathcal{C}^{0}(M)$ like in Theorem 2.2 of [13] and, using Theorem 4.1, prove them in exactly the same way.

THEOREM 4.4. - For sufficiently small $\vartheta, \varepsilon$ and large $c_{g}$ there is a $\kappa \in(0,1)$ such that for all nonempty $\Lambda_{1}, \Lambda_{2} \in \mathcal{F}$ the following holds with the constant $c\left(\Lambda_{1}, \Lambda_{2}, \kappa\right) \stackrel{\text { def }}{=}$ $\kappa^{\left.-\max \left\{\|p-q\|: p \in \Lambda_{1}, q \in \Lambda_{2}\right\}\right)}$ and some positive constants $c_{19}, c_{20}$ :

1. If $\psi_{1} \in \mathcal{C}\left(\left(S^{1}\right)^{\Lambda_{1}}\right)$ and $\psi_{2} \in \mathcal{C}\left(\left(S^{1}\right)^{\Lambda_{2}}\right)$ then

$$
\begin{aligned}
& \left|\int_{M} \mathrm{~d} \nu^{*} \psi_{1} \psi_{2}-\left(\int_{M} \mathrm{~d} \nu^{*} \psi_{1}\right)\left(\int_{M} \mathrm{~d} \nu^{*} \psi_{2}\right)\right| \\
& \quad \leqslant c_{19} \vartheta^{-\left|\Lambda_{1}\right|-\left|\Lambda_{2}\right|}\left\|\psi_{1}\right\|_{\infty}\left\|\psi_{2}\right\|_{\infty} \kappa^{\operatorname{dist}\left(\Lambda_{1}, \Lambda_{2}\right)} .
\end{aligned}
$$

2. If $\psi_{1} \in \mathcal{C}\left(\left(S^{1}\right)^{\Lambda_{1}}\right)$ and $\psi_{2} \in \mathcal{H} \cap \mathcal{C}\left(\left(S^{1}\right)^{\Lambda_{2}}\right)$ then

$$
\begin{aligned}
& \left|\int_{M} \mathrm{~d} \nu^{*} K_{S}^{T}\left(\psi_{1} \circ \tau\right) \psi_{2}-\left(\int_{M} \mathrm{~d} \nu^{*} \psi_{1} \circ \tau\right)\left(\int_{M} \mathrm{~d} \nu^{*} \psi_{2}\right)\right| \\
& \quad \leqslant c\left(\Lambda_{1}, \Lambda_{2}, \kappa\right) c_{20}^{\left|\Lambda_{1}\right|+\left|\Lambda_{2}\right|}\left\|\psi_{1}\right\|_{\infty}\left\|\psi_{2}\right\|_{\Lambda_{2}} \kappa^{m(\tau)} \exp \left(-c_{21} T\right) .
\end{aligned}
$$

3. If the system is translation-invariant and $\psi_{1}, \psi_{2} \in \mathcal{C}(M)$ then

$$
\lim _{\max \{m(\tau), T\} \rightarrow \infty} \int_{M} \mathrm{~d} \nu^{*} K_{S}^{T}\left(\psi_{1} \circ \tau\right) \psi_{2}=\left(\int_{M} \mathrm{~d} \nu^{*} \psi_{1}\right)\left(\int_{M} \mathrm{~d} \nu^{*} \psi_{2}\right) .
$$

\section{Acknowledgements}

This paper is based on a part of my thesis [12]. I would like to thank my supervisor Hans Henrik Rugh for his great support, and also Roger Tribe for many helpful discussions and suggestions. Further, I thank Viviane Baladi and Véronique MaumeDeschamps for helpful discussions and the referee for many useful comments and 
corrections. Finally, I thank the EC for financial support through the TMR-Fellowship ERBFMBICT-961157.

\section{REFERENCES}

[1] Arnold L., Random Dynamical Systems, Springer, 1998.

[2] Baladi V., Degli Esposti M., Isola S., Järvenpää E., Kupiainen A., The spectrum of weakly coupled map lattices, J. Math. Pures et Appl. 77 (1998) 539-584.

[3] Bauer H., Wahrscheinlichkeitstheorie, 4th edn., de Gruyter, 1991.

[4] Bricmont J., Kupiainen A., Coupled analytic maps, Nonlinearity 8 (1995) 379-396.

[5] Bricmont J., Kupiainen A., High temperature expansions and dynamical systems, Comm. Math. Phys. 178 (1996) 703-732.

[6] Bricmont J., Kupiainen A., Infinite dimensional SRB-measures. Lattice dynamics (Paris, 1995), Physica D 103 (1997) 1-4, 18-33.

[7] Bunimovich L.A., Coupled map lattices: One step forward and two steps back, Physica D 86 (1995) 248-255.

[8] Bunimovich L.A., Sinai Y.G., Space-time chaos in coupled map lattices, Nonlinearity 1 (1988) 491-516.

[9] Dobrushin R.L., Markov processes with a large number of locally interacting components: existence of a limit process and its ergodicity, Problems Inform. Transmission 7 (1971) 149-164.

[10] Dobrushin R.L., Markov processes with many locally interacting components - the reversible case and some generalizations, Problems Inform. Transmission 7 (1971) 235 241.

[11] Dobrushin R.L., Sinai Ya.G. (Eds.), Multicomponent Random Systems, Advances in Probability and Related Topics, Vol. 6, 1980 (originally published in Russian).

[12] Fischer T., Transfer operators for deterministic and stochastic coupled map lattices, Thesis, University of Warwick, 1998.

[13] Fischer T., Rugh H.H., Transfer operators for coupled analytic maps, Ergodic Theory Dynamical Systems 20 (2000) 109-143.

[14] Glauber R.J., Time-dependent statistics of the Ising model, J. Math. Phys. 4 (1963) 294-307.

[15] Harris T.E., Nearest-neighbor Markov interaction processes on multidimensional lattices, Adv. Math. 9 (1972) 66-89.

[16] Holley R., A class of interactions in an infinite particle system, Adv. Math. 5 (1970) 291309.

[17] Jiang M., Equilibrium states for lattice models of hyperbolic type, Nonlinearity 8 (5) (1994) 631-659.

[18] Jiang M., Ergodic properties of coupled map lattices of hyperbolic type, Penns. State University Dissertation, 1995.

[19] Jiang M., Mazel A., uniqueness of Gibbs states and exponential decay of correlation for some lattice models, J. Statist. Phys. 82 (3-4) (1995).

[20] Jiang M., Pesin Ya.B., Equilibrium measures for coupled map lattices: existence, uniqueness and finite-dimensional approximations, CMP 193 (1998) 675-711.

[21] Keller G., Künzle M., Transfer operators for coupled map lattices, Ergodic Theory Dynamical Systems 12 (1992) 297-318.

[22] Lang S., Real and Functional Analysis, 3rd edn., Springer, 1993.

[23] Liggett T.M., Existence theorems for infinite particle systems, Trans. Amer. Math. Soc. 165 (1972) 471-481. 
[24] Liggett T.M., Interacting Particle Systems, Grundlehren der mathematischen Wissenschaften, Vol. 276, Springer, 1985.

[25] Maes C., Van Moffaert A., Stochastic stability of weakly coupled lattice maps, Nonlinearity 10 (1997) $715-730$.

[26] Pesin Ya.B., Sinai Ya.G., Space-time chaos in chains of weakly interacting hyperbolic mappings, Adv. Soviet Math. 3 (1991) 165-198.

[27] Spitzer F., Random processes defined through the interaction of an infinite particle system, in: Springer Lecture Notes in Mathematics, Vol. 89, Springer, 1969, pp. 201-223.

[28] Spitzer F., Interaction of Markov processes, Adv. Math. 5 (1970) 246-290.

[29] Volevich D.L., The Sinai-Bowen-Ruelle measure for a multidimensional lattice of interacting hyperbolic mappings, Russ. Akad. Dokl. Math. 47 (1993) 117-121.

[30] Volevich D.L., Construction of an analogue of Bowen-Ruell-Sinai measure for a multidimensional lattice of interacting hyperbolic mappings, Russ. Akad. Math. Sbornik 79 (1994) 347-363. 